\title{
COXETER FUNCTORS WITHOUT DIAGRAMS
}

\author{
BY \\ MAURICE AUSLANDER ${ }^{1}$, MARIA INÉS PLATZECK ${ }^{2}$ AND IDUN REITEN \begin{abstract}
notion of partial Coexeter functor from diagrams to certain types of artin rings and artin algebras. The rest of the paper is devoted to a discussion of the connection between the various Coxeter functors which exist for diagrams as well as for artin rings and artin algebras.
\end{abstract} \\ ABSTRACT. The first part of this paper is devoted to a generalization of the
}

Introduction. For the study of the representation theory of hereditary artin algebras $\Lambda$ in [9], [13], [17], in particular for the investigation of when $\Lambda$ has only a finite number of nonisomorphic indecomposable finitely generated modules, certain types of functors between module categories over hereditary algebras called partial Coxeter functors and Coxeter functors played an important role.

In this paper we develop a theory of partial Coxeter functors for an artin algebra $\Lambda$ with a simple projective noninjective module $S$. We shall not recall the definition of the partial Coxeter functors in the diagramatic setting here (see $\S 5$ ), but it is shown in [13] that such a functor $S^{+}$from $\bmod \Lambda$ to $\bmod \Lambda^{\prime}$, where $\Lambda$ and $\Lambda^{\prime}$ are hereditary tensor algebras and $\bmod \Lambda$ is the category of finitely generated (left) $\Lambda$-modules, is left exact and has the following properties. (i) There is a simple projective noninjective $\Lambda$-module $S$ and a simple injective nonprojective $\Lambda^{\prime}$-module $T$ such that $S^{+}(S)=0$ and $S^{+}$induces an equivalence of categories between the full subcategory of $\bmod \Lambda$ whose objects are the $A$ in $\bmod \Lambda$ such that $S$ is not a summand of $A$, and the full subcategory of $\bmod \Lambda^{\prime}$ whose objects are the $A$ in $\bmod \Lambda^{\prime}$ such that $T$ is not a summand of $A$. (ii) If $0 \rightarrow A \rightarrow B \rightarrow C \rightarrow 0$ is an exact sequence in mod $\Lambda$ and $A$ has no summand isomorphic to $S$, then $0 \rightarrow S^{+}(A)$ $\rightarrow S^{+}(B) \rightarrow S^{+}(C) \rightarrow 0$ is exact. For left artin rings $\Lambda$ and $\Lambda^{\prime}$, we shall call a functor from $\bmod \Lambda$ to $\bmod \Lambda^{\prime}$ with property (i) a left partial Coxeter functor, and show that it must be left exact and have property (ii).

Let $\Lambda$ be an artin algebra. We will denote by $D$ the ordinary duality for artin algebras, and by $\operatorname{Tr} M$ the transpose of the $\Lambda$-module $M$, that is the $\Lambda^{\text {op }}$-module defined by the exact sequence $\operatorname{Hom}_{\Lambda}\left(Q_{0}, \Lambda\right) \rightarrow \operatorname{Hom}_{\Lambda}\left(Q_{1}, \Lambda\right) \rightarrow$

Received by the editors September 14, 1977.

AMS (MOS) subject classifications (1970). Primary 16A46, 16A64; Secondary 16A62.

${ }^{1}$ Supported by N.S.F. Grant PCM 77-04951.

${ }^{2}$ Supported by N.S.F. Grant MCS 77-03478. 
Tr $M \rightarrow 0$, induced by the minimal projective presentation $Q_{1} \rightarrow Q_{0} \rightarrow M \rightarrow$ 0 . Let $\bmod \Lambda$ and $\overline{\bmod } \Lambda$ denote, respectively, the category of finitely generated modules modulo projectives and the category of finitely generated modules modulo injectives. We recall that $\operatorname{Tr}$ induces a duality $\operatorname{Tr}: \bmod \Lambda$ $\rightarrow \bmod \Lambda^{\mathrm{op}}$, and $D \operatorname{Tr}: \bmod \Lambda \rightarrow \overline{\bmod } \Lambda$ is an equivalence of categories (see [6, §2]). When $\Lambda$ is hereditary then $\bmod \Lambda$ is just the full subcategory $\bmod _{P} \Lambda$ of $\bmod \Lambda$, whose objects are the modules $A$ with no nonzero projective summands and $\overline{\bmod } \Lambda$ is the full subcategory $\bmod _{I} \Lambda$ of $\bmod \Lambda$ whose objects are the modules $A$ with no nonzero injective summands. So in this case $D$ Tr defines a functor from $\bmod \Lambda$ to itself (see [5, \$2]).

Now let $\Lambda$ be a basic artin algebra and let $S$ be a simple projective noninjective $\Lambda$-module. Let $P$ be defined by $\Lambda=S \amalg P, X=P \amalg \operatorname{Tr} D S$ and $\Gamma=\operatorname{End}_{\Lambda}(X)^{\mathrm{op}}$. In $\S 1$ we study the functor $F=\operatorname{Hom}_{\Lambda}(X):, \bmod \Lambda \rightarrow$ mod $\Gamma$. Under the assumption that $\operatorname{Hom}_{\Lambda}(\operatorname{Tr} D S, \Lambda)$ is zero we show that $F$ is a left partial Coxeter functor. Since the partial Coxeter functors have turned out to be useful in the study of the representation theory of hereditary tensor algebras, it is hoped that these partial Coxeter functors, or more generally, the functor $F=\operatorname{Hom}_{\Lambda}(X$,$) , even when \operatorname{Hom}(\operatorname{Tr} D S, \Lambda)$ is not zero, should be useful. They have already been successfully applied by R. Bautista in [8], in his study of algebras where every map between indecomposable projectives is zero or a monomorphism. Clearly, if $F=\operatorname{Hom}(X$,$) is a partial Coxeter$ functor, then $\Lambda$ has only a finite number of nonisomorphic indecomposable modules if and only if $\Gamma$ does, whereas Bautista has shown that this is not true in general. However, it would be interesting to know when this is true, and also to know the class of algebras $\Lambda$ such that after applying a finite number of functors of the above type we reach an hereditary algebra, since much is known about the representation theory of hereditary algebras.

The method we use is the theory of almost split sequences. We recall from [6, §4], that for an artin ring $\Lambda$, an exact sequence $0 \rightarrow A \stackrel{f}{\rightarrow} B \stackrel{g}{\rightarrow} C \rightarrow 0$ in $\bmod \Lambda$ is said to be almost split if it is not split, $A$ and $C$ are indecomposable and in addition it has the following properties. Given any map $h: U \rightarrow C$ which is not a splittable epimorphism, there is a map $t: U \rightarrow B$ such that $g t=h$, and given any map $h: A \rightarrow V$ which is not a splittable monomorphism, there is a map $s: B \rightarrow V$ such that $s f=h$. It follows from the proof of Proposition 4.1 in [6] that a nonsplit exact sequence $0 \rightarrow A \stackrel{f}{\rightarrow} B \rightarrow C \rightarrow 0$ with $A$ and $C$ indecomposable is almost split if and only if given a map $h$ : $A \rightarrow V$ which is not a split monomorphism, there is a map $t: B \rightarrow V$ such that tf $=h$. For an artin algebra $\Lambda$ existence and uniqueness of an almost split sequence $0 \rightarrow A \rightarrow B \rightarrow C \rightarrow 0$ for every indecomposable nonprojective $\Lambda$ module $C$ (every indecomposable noninjective $\Lambda$-module $A$ ) has been proven in [6, Proposition 4.3]. It has also been proven there for artin algebras that if 
the sequence $0 \rightarrow A \rightarrow B \rightarrow C \rightarrow 0$ is almost split then $A \cong D \operatorname{Tr} C$.

In $\S 1$ we only need the existence of an almost split sequence $0 \rightarrow A \rightarrow B \rightarrow$ $C \rightarrow 0$ when the module $A$ is simple projective. We also need that in this case $B$ is a projective $\Lambda$-module. These properties are known for artin algebras (see [6, Theorem 5.5]). At the end of $\$ 1$ we give an independent proof of the existence of the almost split sequence $0 \rightarrow A \rightarrow B \rightarrow C \rightarrow 0$ and of the fact that $B$ is projective for the special case when $A$ is simple projective noninjective, and for this we only need to assume that $\Lambda$ is an artin ring that is not necessarily an artin algebra.

In $\$ 2$ we study the notion of left partial Coxeter functors between left artin rings defined above, and also the notion of right partial Coxeter functors. We show that both the left and right partial Coxeter functors are unique up to "equivalence" of functors in the following sense. For left artin rings $\Lambda_{1}, \Lambda_{1}^{\prime}$, $\Lambda_{2}, \Lambda_{2}^{\prime}$ we say that two functors $F_{1}: \bmod \Lambda_{1} \rightarrow \bmod \Lambda_{1}^{\prime}, F_{2}: \bmod \Lambda_{2} \rightarrow$ $\bmod \Lambda_{2}^{\prime}$ are equivalent if there are equivalences of categories $u: \bmod \Lambda_{1} \rightarrow$ $\bmod \Lambda_{2}$ and $v: \bmod \Lambda_{1}^{\prime} \rightarrow \bmod \Lambda_{2}^{\prime}$ such that $v F_{1}=F_{2} u$. We also show, up to equivalence, what the left partial Coxeter functors have to look like if the first ring is artin. In particular, the left and right partial Coxeter functors studied in $\$ 1$ for artin algebras are the only ones up to equivalence.

In $\$ 4$ we study certain functors between the module categories over generalized triangular matrix rings. Specializing our assumptions, our functors will be partial Coxeter functors. Using the results of $\$ 2$ we can then describe all left partial Coxeter functors from an artin ring to a left artin ring. As we pointed out before, the partial Coxeter functors defined in [9], [13] using diagrams, and also the ones defined in [14] fit into our general theory of partial Coxeter functors. By our uniqueness result we get a strong relationship between these various cases.

In [9] and [13] a Coxeter functor $C^{+}$from $\bmod \Lambda$ to $\bmod \Lambda^{\prime}$, for an hereditary tensor ring $\Lambda$ with duality conditions (see $\S 5$ for definition), is defined as a composite of partial Coxeter functors (see $\$ 5$ ). In $\$ 3$ we show for an hereditary artin algebra $\Lambda$, not necessarily a tensor algebra, how to construct ring isomorphisms $\phi: \Lambda \rightarrow \Lambda_{n}$ and a composition of partial Coxeter functors $F_{n} \cdots F_{1}: \bmod \Lambda \rightarrow \bmod \Lambda_{n}$ such that $\bar{\phi} F_{n} \cdots F_{1}=D \mathrm{Tr}$, where $\bar{\phi}: \bmod \Lambda_{n} \rightarrow \bmod \Lambda$ is the equivalence of categories induced by the isomorphism $\phi: \Lambda \rightarrow \Lambda_{n}$. This does not mean, however, that a given Coxeter functor $C^{+}$is isomorphic to the functor $D$ Tr for hereditary tensor algebras. In fact, it is not possible to prove this, because we show that $C^{+}$is not uniquely defined up to isomorphism, since it depends on the choice of certain bimodule isomorphisms. However, it has been proven in [10] that there is a special choice of these isomorphisms such that the corresponding functor $C^{+}$is isomorphic to $D \mathrm{Tr}$. 
The connections of our work with the diagramatic results of [9] and [13] are studied in $\$ 5$. We also prove in this section that for an artin hereditary tensor ring with duality conditions any Coxeter functor $C^{+}$is isomorphic to $D_{1} \mathrm{Tr}$, for some duality $D_{1}$, but there might be dualities $D_{1}$ such that $D_{1} \operatorname{Tr}$ is not isomorphic to any Coxeter functor $C^{+}$.

We shall only be dealing with basic left artin rings. This is no loss of generality since every left artin ring is Morita equivalent to a basic left artin ring. Further we have that if two basic left artin rings are Morita equivalent, they are isomorphic.

1. Functors and endomorphism rings. Throughout this paper we shall assume that all artin rings are basic, that is, the ring modulo its radical $\mathbf{r}$ is a product of division rings.

Let $\Lambda$ be an artin algebra, that is, an artin ring which is a finitely generated module over its center, which is also an artin ring, and denote by mod $\Lambda$ the category of finitely generated (left) $\Lambda$-modules. Assume that $\Lambda$ has a simple projective module $S$ which is not injective and denote by $P$ the direct sum of one copy of each of the other indecomposable $\Lambda$-modules. Let further $X=\operatorname{Tr} D S \amalg P$, where $D$ denotes the ordinary duality between $\bmod \Lambda$ and $\bmod \Lambda^{\mathrm{op}}$, and $\operatorname{Tr}$ denotes the transpose. Let $\Gamma=\operatorname{End}_{\Lambda}(X)^{\mathrm{op}}$, and denote by $F$ the functor $\operatorname{Hom}_{\Lambda}(X)=,(X$,$) from \bmod \Lambda$ to $\bmod \Gamma$. Denote by $\mathrm{e}=\mathrm{e}_{s}$ the full subcategory of $\bmod \Lambda$ whose objects are the $A$ in $\bmod \Lambda$ such that $\operatorname{Hom}_{\Lambda}(A, S)=0$, in other words the $A$ in $\bmod \Lambda$ having no summand isomorphic to $S$. We shall also let $F$ denote the restriction functor from $C$ to mod $\Gamma$. For a simple injective nonprojective $\Gamma$-module $T$, denote by $\mathscr{D}=\mathscr{D}_{T}$ the full subcategory of $\bmod \Gamma$ whose objects are the $B$ in mod $\Gamma$ such that $\operatorname{Hom}_{\Gamma}(T, B)$ is zero, that is, the $B$ in mod $\Gamma$ having no summand isomorphic to $T$.

In this section we shall study the functor $F: \bmod \Lambda \rightarrow \bmod \Gamma$, and relationships between the rings $\Lambda$ and $\Gamma$. We also study a certain type of right exact functors $G=Y \otimes: \bmod \Gamma \rightarrow \bmod \Lambda$, having properties "dual" to the properties of $F$.

More specifically, we show that $F: \mathcal{C} \rightarrow \bmod \Gamma$ is a fully faithful functor. Under the assumption that $(\operatorname{Tr} D S)^{*}=\operatorname{Hom}_{\Lambda}(\operatorname{Tr} D S, \Lambda)$ is zero, we show that $T=\operatorname{Ext}_{\Lambda}^{1}(X, S)$ is a simple injective $\Gamma$-module and that $F$ induces an equivalence of categories between $\mathcal{C}_{S}$ and $\mathscr{D}_{T}$. We go on to study some of the relationships between the algebras $\Lambda$ and $\Gamma$. We show that if $\Lambda$ is hereditary then $\Gamma$ is. Actually, we show more generally that gl. $\operatorname{dim} . \Lambda-1<\operatorname{gl} . \operatorname{dim} . \Gamma$ $\leqslant$ gl. $\operatorname{dim} . \Lambda$ and that $\mathrm{gl} . \operatorname{dim} . \Lambda=$ gl. $\operatorname{dim} . \Gamma$ if $\operatorname{id}_{\Lambda} S<\operatorname{gl} . \operatorname{dim} . \Lambda$, where $\mathrm{id}_{\Lambda} S$ denotes the injective dimension of $S$, or if $(\operatorname{Tr} D S)^{*}=0$. We also show that if $(\operatorname{Tr} D S)^{*}=0$, then $\Lambda$ is a factor of an hereditary algebra if and only if $\Gamma$ is. 
We end the section with a construction of an almost split sequence $0 \rightarrow S \rightarrow Q \rightarrow K \rightarrow 0$, when $S$ is a simple projective noninjective module over an artin ring $\Lambda$, which is not necessarily an artin algebra.

We start out with some preliminary results.

LemMa 1.1. Let $A$ be in $\bmod \Lambda$. Then $\operatorname{Ext}_{\Lambda}^{1}(\operatorname{Tr} D S, A)=0$ if and only if $A$ is in $\mathcal{C}_{s}=\mathcal{C}$.

Proof. This follows directly from [6]. However, for the convenience of the reader we outline a proof. Let $A$ be indecomposable in $\bmod \Lambda$. If $A=S$, we have an almost split sequence $0 \rightarrow S \rightarrow E \rightarrow \operatorname{Tr} D S \rightarrow 0$, so that $\operatorname{Ext}^{1}(\operatorname{Tr} D S, S) \neq 0$. Assume that $A$ is not isomorphic to $S$, and assume that there is some nonsplit exact sequence $0 \rightarrow A \rightarrow B \rightarrow \operatorname{Tr} D S \rightarrow 0$. Then we have a commutative diagram

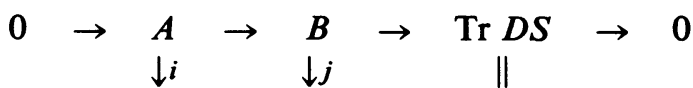

$$
\begin{aligned}
& 0 \rightarrow S \rightarrow E \rightarrow \operatorname{Tr} D S \rightarrow 0
\end{aligned}
$$

where the bottom sequence is almost split. Since $i$ must be zero because $S$ is simple projective, it follows that the bottom sequence splits, which is a contradiction.

LemMa 1.2. Let $0 \rightarrow A \rightarrow B \rightarrow C \rightarrow 0$ be an exact sequence in $\bmod \Lambda$.

(a) If $A$ is in $\mathcal{C}$, then $0 \rightarrow(X, A) \rightarrow(X, B) \rightarrow(X, C) \rightarrow 0$ is exact.

(b) If $B$ is in $\mathcal{C}$ and $0 \rightarrow(X, A) \rightarrow(X, B) \rightarrow(X, C) \rightarrow 0$ is exact, then $A$ is in e.

Proof. Consider the long exact sequence $0 \rightarrow(X, A) \rightarrow(X, B) \rightarrow(X, C) \rightarrow$ $\operatorname{Ext}_{\Lambda}^{1}(X, A) \rightarrow \operatorname{Ext}_{\Lambda}^{1}(X, B)$. By Lemma 1.1, $\operatorname{Ext}_{\Lambda}^{1}(X, A)$ is zero, and $B$ is in $\mathcal{C}$ if and only if $\operatorname{Ext}_{\Lambda}^{1}(X, B)$ is zero. Hence both (a) and (b) follow.

LEMMA 1.3. Let $\Lambda$ be an artin algebra, $Y$ some finitely generated $\Lambda$-module, $\Gamma=\operatorname{End}_{\Lambda}(Y)^{\mathrm{op}}$, and $F=(Y):, \bmod \Lambda \rightarrow \bmod \Gamma$.

(a) $F$ induces an equivalence of categories between $\operatorname{add}(Y)$, whose objects are the summands of finite sums of copies of $Y$, and the category of finitely generated projective $\Gamma$-modules.

(b) The restriction of $F$ to the full subcategory of $\bmod \Lambda$ whose objects are the $M$ such that there is an exact sequence $Y_{1} \rightarrow Y_{0} \rightarrow M \rightarrow 0$, with $Y_{0}, Y_{1}$ in $\operatorname{add}(Y)$ with the property that $\left(Y, Y_{0}\right) \rightarrow\left(Y, Y_{1}\right) \rightarrow(Y, M) \rightarrow 0$ is exact, is fully faithful.

See $[4, \S 1]$ and $[2$, Proposition 3.2] for the proof.

We can now prove the following.

Proposition 1.4. Let the notation be as given before the previous lemmas. Then $F: \mathcal{C} \rightarrow \bmod \Gamma$ is a fully faithful functor. 
Proof. By Lemma 1.3 it is enough to show that if $M$ is in $\mathcal{C}$, then there is an exact sequence $X_{2} \rightarrow X_{1} \rightarrow M \rightarrow 0$ with $X_{2}, X_{1}$ in $\operatorname{add}(X)$, such that $F\left(X_{2}\right) \rightarrow F\left(X_{1}\right) \rightarrow F(M) \rightarrow 0$ is exact.

Since $M$ is in $\mathcal{C}$, it is clear that the projective cover $P$ of $M$ has no summand isomorphic to $S$. Hence we have some epimorphism $f: X^{\prime} \rightarrow M$ with $X^{\prime}$ in $\operatorname{add}(X)$. It is easy to see, by using that $\operatorname{Hom}_{\Lambda}(X, M)$ is a finitely generated module over the center of $\Lambda$, that we have some epimorphism $f$ : $X_{1} \rightarrow M$ with $X_{1}$ in $\operatorname{add}(X)$ such that $F\left(X_{1}\right) \rightarrow F(M) \rightarrow 0$ is exact. By Lemma 1.2(b) we have that, since $X_{1}$ is in $\mathcal{C}, K=\operatorname{Ker}(f)$ is in $\mathcal{C}$. As above we choose an epimorphism $g: X_{2} \rightarrow K$ with $X_{2}$ in $\operatorname{add}(X)$, such that $F\left(X_{2}\right) \rightarrow F(K) \rightarrow 0$ is exact. Then $X_{2} \rightarrow X_{1} \rightarrow M \rightarrow 0$ has the property that $F\left(X_{2}\right) \rightarrow F\left(X_{1}\right) \rightarrow$ $F(M) \rightarrow 0$ is exact, by Lemma $1.3(\mathrm{~b})$. This finishes the proof of the proposition.

To prove a dual result we introduce the following notation. Assume that a basic artin algebra $\Gamma$ has a simple injective module $T$ which is not projective. Let $I$ denote the direct sum of one copy of each of the other indecomposable injective $\Gamma$-modules. Let $Y$ denote the $\Gamma^{\mathrm{op}}$-module $D(I) \amalg \operatorname{Tr}(T)$, and let $\Lambda=\operatorname{End}(Y)$. Denote by $G$ the functor $Y \otimes \quad$ from $\bmod \Gamma$ to $\bmod \Lambda$, and also its restriction to $\mathscr{D}_{T}=\mathscr{D}$. We then have the following.

Proposition 1.5. Let the notation be as above. Then $G: \mathscr{D}_{T} \rightarrow \bmod \Lambda$ is a fully faithful functor.

Proof. Let $F: \bmod \Gamma^{\text {op }} \rightarrow \bmod \Lambda^{\text {op }}$ be the functor $\operatorname{Hom}_{\Gamma \text { op }}(\operatorname{Tr} T \amalg D(I)$, ). Here $\operatorname{Tr} T=\operatorname{Tr} D(D T)$, where $D(T)$ is a simple projective $\Gamma^{\mathrm{op}}$-module and $D(I)$ is the direct sum of one copy of each of the other indecomposable projective $\Gamma^{\text {op }}$-modules. Let $M$ be in $\bmod \Gamma$. By [11, p. 119], we have a natural isomorphism

$$
\begin{aligned}
D G(M) & =D\left((\operatorname{Tr} T \amalg D(I)) \otimes_{\Gamma} M\right) \\
& \cong \operatorname{Hom}_{\Gamma}(\operatorname{Tr} T \amalg D(I), D(M))=F D(M) .
\end{aligned}
$$

If we consider this isomorphism an identification, we then have a commutative diagram:

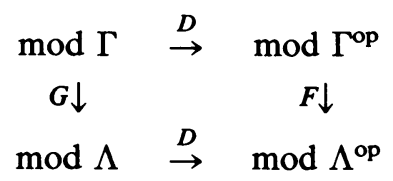

Since we know from Proposition 1.4 that $F: \bigodot_{D(T)} \rightarrow \bmod \Lambda^{\text {op }}$ is fully faithful, it follows that $G: \mathscr{D}_{T} \rightarrow \bmod \Lambda$ is fully faithful.

Before we give the main result of this section we need some more preliminary results. 
LEMMA 1.6. Let $0 \rightarrow S \stackrel{g}{\rightarrow} Q \stackrel{f}{\rightarrow} \operatorname{Tr} D S \rightarrow 0$ be the almost split sequence in $\bmod \Lambda$. Then:

(a) $f$ is a projective cover.

(b) The induced sequence of $\Gamma$-modules $0 \rightarrow(X, Q) \rightarrow(X, \operatorname{Tr} D S) \rightarrow$ $\operatorname{Ext}^{1}(X, S) \rightarrow 0$ is exact and is a minimal projective presentation for $\operatorname{Ext}_{\Lambda}^{1}(X, S)$.

(c) $T=\operatorname{Ext}_{\Lambda}^{1}(X, S)$ is a simple $\Gamma$-module.

Proof. Since $S$ is simple projective we know from [6, Theorem 5.5], that we have an almost split sequence $0 \rightarrow S \rightarrow Q \rightarrow \operatorname{Tr} D S \rightarrow 0$, where $Q \rightarrow \operatorname{Tr} D S$ is a projective cover. We also know from [7, Theorem 2.4], that in an almost split sequence no end term is isomorphic to a summand of the middle term. Hence $S$ is not isomorphic to a summand of $Q$. By Lemma 1.1 we then have $\operatorname{Ext}_{\Lambda}^{1}(\operatorname{Tr} D S, Q)=0$, and consequently $\operatorname{Ext}_{\Lambda}^{1}(X, Q)=0$. Since also $(X, S)=$ 0 , we have the exact sequence $0 \rightarrow(X, Q) \stackrel{s}{\rightarrow}(X, \operatorname{Tr} D S) \stackrel{t}{\rightarrow} \operatorname{Ext}_{\Lambda}^{1}(X, S) \rightarrow$ 0 . Since $0 \rightarrow S \rightarrow Q \rightarrow \operatorname{Tr} D S \rightarrow 0$ is almost split, we have from [6, Proposition 3.1], that $\operatorname{Ext}_{\Lambda}^{1}(X, S)$ is a simple $\Gamma$-module. The given projective resolution of $T$ is minimal because $(X, \operatorname{Tr} D S)$ is indecomposable. Therefore (a), (b) and (c) have been proven.

LEMMA 1.7. If $(\operatorname{Tr} D S)^{*}=0$, then $\operatorname{End}(\operatorname{Tr} D S)^{\mathrm{op}}$ is a division algebra.

Proof. Let $0 \rightarrow S \stackrel{f}{\rightarrow} Q \stackrel{g}{\rightarrow} \operatorname{Tr} D S \rightarrow 0$ be an almost split sequence. Assume that there is a nonzero morphism $h: \operatorname{Tr} D S \rightarrow \operatorname{Tr} D S$ which is not an isomorphism. Then there would be a nonzero morphism $t: \operatorname{Tr} D S \rightarrow Q$ such that $g t=h$. Since $(\operatorname{Tr} D S)^{*}=0$ and $Q$ is projective, we have a contradiction. Hence every nonzero morphism $h: \operatorname{Tr} D S \rightarrow \operatorname{Tr} D S$ is an isomorphism, so that End(Tr $D S)^{\text {op }}$ is a division algebra.

LeMma 1.8. Assume that $(\operatorname{Tr} D S)^{*}=0$. If $\alpha:(X, \operatorname{Tr} D S) \rightarrow\left(X, X_{1}\right)$, with $X_{1}$ in $\operatorname{add}(X)$, is a nonzero morphism, then $\alpha$ is a split monomorphism.

Proof. Since $X_{1}$ is in $\operatorname{add}(X)$, we have $X_{1}=Q \amalg(\operatorname{Tr} D S)^{r}$, where $Q$ is projective, $r \geqslant 0$ and $(\operatorname{Tr} D S)^{0}=0$. Given $\alpha:(X, \operatorname{Tr} D S) \rightarrow\left(X, X_{1}\right)$, there is by Lemma 1.3 a morphism $\alpha_{1}: \operatorname{Tr} D S \rightarrow X_{1}=Q \amalg(\operatorname{Tr} D S)^{r}$ in $\bmod \Lambda$ such that $\left(X, \alpha_{1}\right)=\alpha$. Since $(\operatorname{Tr} D S)^{*}=0$, we have $\operatorname{Im} \alpha_{1} \subseteq(\operatorname{Tr} D S)^{r}$. There is then a morphism $(\operatorname{Tr} D S)^{r} \rightarrow \operatorname{Tr} D S$ such that the composite morphism $\operatorname{Tr} D S \rightarrow(\operatorname{Tr} D S)^{r} \rightarrow \operatorname{Tr} D S$ is not zero. Since, by Lemma 1.7, End $(\operatorname{Tr} D S)^{\text {op }}$ is a division algebra, $\alpha_{1}: \operatorname{Tr} D S \rightarrow(\operatorname{Tr} D S)^{r}$ is a split monomorphism, so that $\alpha=\left(X, \alpha_{1}\right):(X, \operatorname{Tr} D S) \rightarrow\left(X, X_{1}\right)$ is a split monomorphism.

LEMMA 1.9. If $(\operatorname{Tr} D S)^{*}=0$, then the simple $\Gamma$-module $T=\operatorname{Ext}^{1}(X, S)$ is injective. 
Proof. To prove the lemma it is enough to show that every monomorphism $\alpha: T \rightarrow N$ splits, where $N$ is in $\bmod \Gamma$. So let $\alpha: T \rightarrow N$ be a monomorphism. Let $\left(X, X_{1}\right) \rightarrow N$ be a projective cover, where $X_{1}$ is in $\operatorname{add}(X)$. Then we have a commutative diagram:

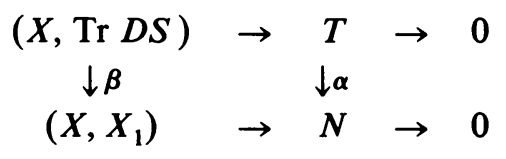

Since $\beta$ is not zero, it is a split monomorphism by Lemma 1.8. Hence the composite morphism $(X, \operatorname{Tr} D S) \rightarrow\left(X, X_{1}\right) \rightarrow N \rightarrow N / \mathbf{r} N$ is not zero, where $\mathbf{r} N$ denotes the radical of the module $N$. This means that $T \rightarrow N \rightarrow N / \mathbf{r} N$ is not zero. It follows that $\alpha$ is a split monomorphism, and this shows that $T$ is injective.

LeMMA 1.10. Let $N$ be indecomposable in $\bmod \Gamma$. Then there is some $M$ in $\bmod \Lambda$ and $a$ nonnegative integer $r$ such that there is an exact sequence $0 \rightarrow T^{r} \rightarrow N \rightarrow(X, M) \rightarrow 0$.

Proof. Let $\left(X, X_{2}\right) \stackrel{s}{\rightarrow}\left(X, X_{1}\right) \rightarrow N \rightarrow 0$ be a minimal projective presentation for $N$ in $\bmod \Gamma$, where $X_{1}$ and $X_{2}$ are in $\operatorname{add}(X)$. Consider the exact sequence $X_{2} \stackrel{h}{\rightarrow} X_{1} \stackrel{g}{\rightarrow} M \rightarrow 0$ in $\bmod \Lambda$, where $h: X_{2} \rightarrow X_{1}$ is such that $F(h)=s$. Let $L=\operatorname{Ker}(g)$ and let $0 \rightarrow K \rightarrow X_{2} \rightarrow L \rightarrow 0$ be exact. Then $0 \rightarrow(X, K) \rightarrow\left(X, X_{2}\right) \stackrel{t}{\rightarrow}(X, L) \rightarrow \operatorname{Ext}_{\Lambda}^{1}(X, K)$ is exact. But by Lemma 1.1, $\operatorname{Ext}_{\Lambda}^{1}(X, K) \cong T^{j}$, where $j$ is the multiplicity of $S$ as a summand of $K$. Hence, $\operatorname{Coker}(t) \cong T^{r}$, for some $r<j$. Since $L$ is clearly in $\mathcal{C}$, we have by Lemma 1.2 an exact sequence $0 \rightarrow(X, L) \rightarrow\left(X, X_{1}\right) \rightarrow(X, M) \rightarrow 0$. We then have a commutative diagram:

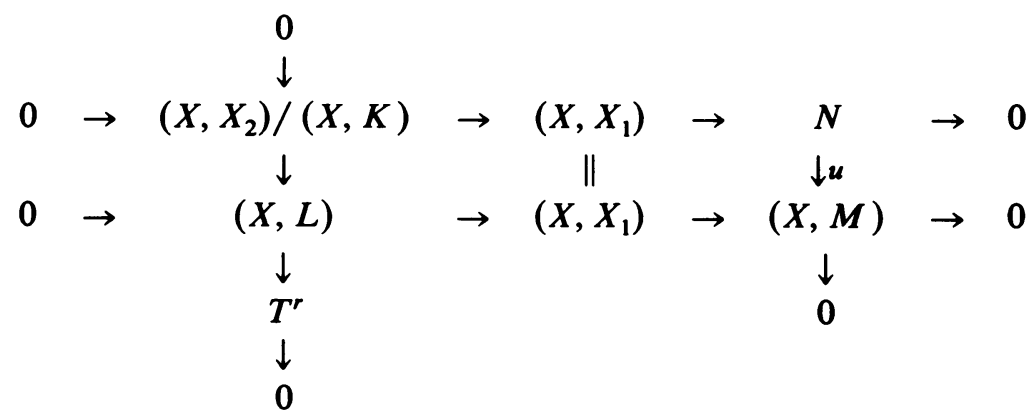

Hence $\operatorname{Ker}(u)=T^{r}$, that is, we have the exact sequence $0 \rightarrow T^{r} \rightarrow$ $N \stackrel{u}{\rightarrow}(X, M) \rightarrow 0$, as desired.

We are now ready to prove the main results of this section. 
THEOREM 1.11. Let $\Lambda$ be a basic algebra with a simple projective noninjective $\Lambda$-module $S$, such that $(\operatorname{Tr} D S)^{*}=0$. Let $X=P \amalg \operatorname{Tr} D S$, where $P$ is given by $\Lambda=P \amalg S$. Let $\Gamma=\operatorname{End}_{\Lambda}(X)^{\mathrm{op}}$ and consider the functor $F=\operatorname{Hom}_{\Lambda}(X$,$) :$ $\bmod \Lambda \rightarrow \bmod \Gamma$. Then $T=\operatorname{Ext}_{\Lambda}^{1}(X, S)$ is a simple injective $\Gamma$-module, and $F$ induces an equivalence of categories between the full subcategory $\mathcal{C}=\mathcal{C}_{S}$ of $\bmod \Lambda$ whose objects are the $A$ in $\bmod \Lambda$ not having $S$ as a summand, and the full subcategory $\mathscr{D}=\mathscr{D}_{T}$ of $\bmod \Gamma$ whose objects are the $B$ in $\bmod \Gamma$ not having $T$ as a summand.

Proof. By Proposition 1.4 we know that the restriction of $F$ to $C$ is fully faithful, and by Lemma 1.9 we know that $T=\operatorname{Ext}_{\Lambda}^{1}(X, S)$ is a simple injective $\Gamma$-module. Let now $N$ be an object in $\mathscr{D}$. By Lemma 1.10 there is then some exact sequence $0 \rightarrow T^{r} \rightarrow N \rightarrow(X, M) \rightarrow 0$. Since $T$ is injective and cannot be a summand of $N$, we have an isomorphism $N \rightarrow(X, M)$. This finishes the proof of the theorem.

THEOREM 1.12. Let $\Gamma$ be a basic artin algebra with a simple injective nonprojective module $T$ such that $(\operatorname{Tr} T)^{*}=0$. Let I denote the sum of one copy of each of the other indecomposable injective $\Gamma$-modules. Let $Y=D(I) \amalg \operatorname{Tr} T$, and $\Lambda=\operatorname{End}_{\Gamma}(Y)$. Then $S=\operatorname{Tor}_{1}^{\Gamma}(Y, T)$ is a simple projective $\Lambda$-module and the functor $G=Y \otimes: \bmod \Gamma \rightarrow \bmod \Lambda$ induces an equivalence of categories between $\mathscr{D}=\mathscr{D}_{T}$ and $\mathrm{C}=\mathrm{C}_{S}$.

Proof. This follows directly from the proof of Proposition 1.5 and from Theorem 1.11. We only need to point out that $\operatorname{Ext}_{\Gamma}^{1}(Y, D T)$ is a simple injective $\Lambda^{\mathrm{op}}$-module and that $D\left(\operatorname{Ext}_{\Lambda}^{1}(Y, D T)\right)=\operatorname{Tor}_{1}^{\Gamma}(Y, T)=S$ by $[11, \mathrm{p}$. 120].

We point out that if $\Lambda$ is an hereditary basic artin algebra which is not semisimple, there is always some simple projective noninjective $\Lambda$-module $S$ and, since submodules of projective modules are projective, the condition $(\operatorname{Tr} D S)^{*}=0$ automatically holds. Since this is an important special case of Theorem 1.11 we formulate it as a special result.

Proposition 1.13. Let $\Lambda$ be a basic hereditary artin algebra and $S$ a simple projective noninjective $\Lambda$-module. With the previous notations, $T=\operatorname{Ext}_{\Lambda}^{1}(X, S)$ is a simple injective $\Lambda$-module, and $F=\operatorname{Hom}_{\Lambda}(X):, \bmod \Lambda \rightarrow \bmod \Gamma$ induces an equivalence of categories between $\mathcal{C}_{S}$ and $\mathscr{D}_{T}$. Further, $\Gamma$ is again hereditary.

Proof. In view of our above results, we only need to prove that $\Gamma$ is hereditary. This will be a consequence of the first part of the next lemma, since we know that $\operatorname{pd}_{\Gamma} T=1$.

We shall now investigate the connection between the algebras $\Lambda$ and $\Gamma$ with respect to global dimension and with respect to the property of being a factor of an hereditary artin algebra. 
LEMMA 1.14. Let, as before, $\Lambda$ be a basic artin algebra with a simple projective noninjective module $S, X=P \amalg T r D S, \Gamma=\operatorname{End}_{\Lambda}(X)^{\text {op }}$. Let $M$ be an indecomposable $\Lambda$-module not isomorphic to $S$.

(a) $\operatorname{pd}_{\Lambda} M-1 \leqslant \operatorname{pd}_{\Gamma}(X, M) \leqslant \operatorname{pd}_{\Lambda} M$.

(b) If $\mathrm{id}_{\Lambda} S<\operatorname{pd}_{\Lambda} M$ or if $(\operatorname{Tr} D S)^{*}=0$ and $M$ is not isomorphic to $\operatorname{Tr} D S$, then $\operatorname{pd}_{\Gamma}(X, M)=\operatorname{pd}_{\Lambda} M$.

Proof. (a) For $i \geqslant 1$, let $\Omega^{i} M$ be defined by the exact sequence $0 \rightarrow \Omega^{i} M \rightarrow$ $P_{i-1} \rightarrow \cdots \rightarrow P_{0} \rightarrow M \rightarrow 0$, where $P_{i-1} \rightarrow \cdots \rightarrow P_{0} \rightarrow M \rightarrow 0$ is part of a minimal projective resolution for $M$. Write $\Omega^{0} M=M$. We first prove that if $B$ is a $\Lambda$-module such that $\left(X, \Omega^{1} B\right) \neq 0$, then $\operatorname{pd}(X, B)=\operatorname{pd}\left(X, \Omega^{1} B\right)+1$. To see this, consider the exact sequence $0 \rightarrow \Omega^{1} B \rightarrow P_{0} \rightarrow B \rightarrow 0$. We can clearly assume that $B$ has no nonzero projective summand, and hence $P_{0}$ can have no summand isomorphic to $S$. We then have $\operatorname{Ext}_{\Lambda}^{1}\left(X, P_{0}\right)=0$ by Lemma 1.1, and consequently an exact sequence

$$
0 \rightarrow\left(X, \Omega^{1} B\right) \rightarrow\left(X, P_{0}\right) \rightarrow(X, B) \rightarrow \operatorname{Ext}_{\Lambda}^{1}\left(X, \Omega^{1} B\right) \rightarrow 0 .
$$

This gives rise to the exact sequences

$$
0 \rightarrow\left(X, \Omega^{1} B\right) \rightarrow\left(X, P_{0}\right) \rightarrow N \rightarrow 0
$$

and

$$
0 \rightarrow N \rightarrow(X, B) \rightarrow \operatorname{Ext}_{\Lambda}^{1}\left(X, \Omega^{1} B\right) \rightarrow 0 .
$$

Since by our assumption $\left(X, \Omega^{1} B\right) \neq 0$, it is easy to see, using that $(X$,$) :$ $\mathcal{C} \rightarrow \bmod \Gamma$ is fully faithful, that $0 \rightarrow\left(X, \Omega^{1} B\right) \rightarrow\left(X, P_{0}\right)$ is not a split monomorphism, so that $N$ is not projective, and hence $\operatorname{pd} N=\operatorname{pd}\left(X, \Omega^{1} B\right)+1$. By Lemmas 1.1 and 1.6 we have that if $\operatorname{Ext}_{\Lambda}^{1}\left(X, \Omega^{1} B\right) \neq 0$, then $\operatorname{pd}^{\operatorname{Ext}_{\Lambda}^{1}}\left(X, \Omega^{1} B\right)$ $=1$. Since $\operatorname{pd} N \geqslant 1$, we get that $\operatorname{pd} N=\operatorname{pd}(X, B)$, so that $\operatorname{pd}(X, B)=$ $\operatorname{pd}\left(X, \Omega^{1} B\right)+1$. If $(X, B) \neq 0$ and $\left(X, \Omega^{1} B\right)=0$, we would get $\operatorname{pd}(X, B)<1$.

Assume now that $M$ is an indecomposable $\Lambda$-module not isomorphic to $S$. If $M$ is projective we have $\operatorname{pd}(X, M)=0=\operatorname{pd} M$. Assume now that $\operatorname{pd}_{\Lambda} M$ $=n>1$. For $i<n \Omega^{i} M$ is then not projective, so that $\left(X, \Omega^{i} M\right) \neq 0$. By repeated application of the above we then get that $\operatorname{pd}(X, M)=$ $\operatorname{pd}\left(X, \Omega^{n-1} M\right)+n-1$, and if also $\left(X, \Omega^{n} M\right) \neq 0$, we $\operatorname{get} \operatorname{pd}(X, M)=$ $\operatorname{pd}\left(X, \Omega^{n} M\right)+n=0+n=n$. If $\left(X, \Omega^{n} M\right)=0$, it follows from the above that $\operatorname{pd}\left(X, \Omega^{n-1} M\right) \leqslant 1$, so that $n-1 \leqslant \operatorname{pd}(X, M) \leqslant n$. If $\operatorname{pd} M=\infty$, we clearly have $\left(X, \Omega^{i} M\right) \neq 0$ for all $i$, so that $\operatorname{pd}(X, M)=\operatorname{pd}\left(X, \Omega^{i} M\right)+i>i$ for all $i$, and consequently $\operatorname{pd}(X, M)=\infty$. This finishes the proof of (a).

(b) Let now $M$ be an indecomposable $\Lambda$-module not isomorphic to $S$, so that $(X, M)$ is not zero. Assume that $\operatorname{pd} M=n$ and $\operatorname{pd}(X, M) \neq n$. By the above, we must then have $n \geqslant 1$ and $\left(X, \Omega^{n} M\right)=0$. Since $\Omega^{n} M \neq 0$, we must then have $S \mid \Omega^{n} M$, and hence $\operatorname{Ext}_{\Lambda}^{n}(M, S) \neq 0$. This shows that $\operatorname{id}_{\Lambda} S>n$. 
Further, if $\operatorname{pd}(X, M) \neq n$, we also see by the above that we must have $\operatorname{pd}\left(X, \Omega^{n-1} M\right)=0$. Since $\Omega^{n-1} M$ is not projective, this means that $\operatorname{Tr} D S \mid \Omega^{n-1} M$. If $n>1, \operatorname{Tr} D S$ is a submodule of $P_{n-2}$, and hence $(\operatorname{Tr} D S)^{*}$ $\neq 0$. If $n=1, \operatorname{Tr} D S \cong M=\Omega^{0} M$. This finishes the proof of (b).

We use the above lemma to get the following relationship between the global dimensions of $\Lambda$ and $\Gamma$.

Proposition 1.15. Let $\Lambda$ be a basic artin algebra with a simple projective noninjective module $S$, and let $X, \Gamma, T$ and $F$ be as before.

(a) gl. dim. $\Lambda-1<$ gl. dim. $\Gamma<$ gl. dim. $\Lambda$.

(b) If $\mathrm{id}_{\Lambda} S<$ gl. dim. $\Lambda$ or if $(\operatorname{Tr} D S)^{*}=0$, then gl. dim. $\Lambda=$ gl. $\operatorname{dim}$. $\Gamma$.

Proof. If $N$ is an indecomposable $\Gamma$-module, there is by Lemma 1.10 an exact sequence $0 \rightarrow T^{r} \rightarrow N \rightarrow(X, M) \rightarrow 0$ with $M$ in $\bmod \Lambda$. Since $\operatorname{pd}_{\Gamma} T=$ 1, we have $\operatorname{pd}_{\Gamma} N \leqslant \max \left(1, \operatorname{pd}_{\Gamma}(X, M)\right)$, hence $\operatorname{pd}_{\Gamma} N<\operatorname{pd}_{\Gamma}(X, M)$, for if $(X, M)$ is projective, then $N=(X, M)$. The desired result is then a direct consequence of Lemma 1.14.

An example of an artin algebra $\Lambda$ such that gl. dim. $\Gamma=$ gl. $\operatorname{dim} . \Lambda-1$ is

$$
\Lambda=\left(\begin{array}{lll}
K & 0 & 0 \\
K & K & 0 \\
0 & K & K
\end{array}\right),
$$

where $K$ is a field. Here gl. $\operatorname{dim} . \Lambda=2, S$ is the unique simple projective $\Lambda$-module and

$$
\Gamma=\left(\begin{array}{lll}
K & 0 & 0 \\
K & K & 0 \\
K & K & K
\end{array}\right)
$$

which has global dimension 1 .

We next study the relationship between $\Lambda$ and $\Gamma$ with respect to being factors of hereditary artin algebras. For this we shall use that $\Lambda$ is a factor of an hereditary algebra if and only if the following condition holds. Given an infinite chain $\cdots \rightarrow P_{n} \stackrel{f_{n}}{\rightarrow} P_{n-1} \rightarrow \cdots \rightarrow P_{1} \stackrel{f_{1}}{\rightarrow} P_{0}$ of nonzero morphisms between indecomposable projective modules, there is some $i$ such that $f_{n}$ is an isomorphism for $n>i$ (see [19]).

Proposition 1.16. Let $\Lambda$ be a basic artin algebra with a simple projective noninjective module $S$, and let $X, \Gamma, T$ and $F$ be as before.

(a) If $\Gamma$ is a factor of an hereditary algebra, then $\Lambda$ is.

(b) If $(\operatorname{Tr} D S)^{*}=0$ and $\Lambda$ is a factor of an hereditary algebra, then $\Gamma$ is.

Proof. (a) Assume that $\Gamma$ is a factor of an hereditary artin algebra, and consider a chain $\cdots \rightarrow P_{n} \stackrel{f_{n}}{\rightarrow} P_{n-1} \rightarrow \cdots \rightarrow P_{1} \stackrel{f_{1}}{\rightarrow} P_{0}$ of nonzero maps between indecomposable projective $\Lambda$-modules. We can assume that no $P_{i}$ is 
isomorphic to $S$, since otherwise $f_{n}$ would be an isomorphism for $n>i$. We then have a chain

$$
\cdots \rightarrow\left(X, P_{n}\right) \stackrel{\left(X, f_{n}\right)}{\rightarrow}\left(X, P_{n-1}\right) \rightarrow \cdots \rightarrow\left(X, P_{1}\right) \stackrel{\left(X, f_{1}\right)}{\rightarrow}\left(X, P_{0}\right)
$$

of nonzero maps between indecomposable projective $\Gamma$-modules. Since $\Gamma$ is a factor of an hereditary artin algebra, there is some $i$ such that $\left(X, f_{n}\right)$ is an isomorphism for $n \geqslant i$. Hence $f_{n}$ is an isomorphism for $n \geqslant i$. This finishes the proof of (a).

(b) Assume that $(\operatorname{Tr} D S)^{*}=0$ and that $\Lambda$ is a factor of an hereditary algebra. The proof of this part is similar, using now that there is no nonzero map from $(X, \operatorname{Tr} D S)$ to $(X, Q)$ when $Q$ is an indecomposable projective $\Lambda$-module.

Let the notation be as before Lemma 1.1. It would be interesting to know the connection between the representation theory of $\Lambda$ and $\Gamma$. In particular, when does the fact that one of these algebras is of finite representation type imply that the other one also is. From Proposition 1.4 it follows that if $\Gamma$ is of finite representation type, then $\Lambda$ is, and it follows from Theorem 1.11 that the converse holds if $(\operatorname{Tr} D S)^{*}=0$. On the other hand, Bautista has given an example showing that $\Gamma$ may be of infinite representation type and $\Lambda$ of finite type.

Since a complete classification of when an hereditary artin algebra is of finite representation type is known [13], [3], it would also be interesting to know for which artin algebras $\Lambda$ we reach an hereditary algebra after applying our construction a finite number of times. By the above we know that it is necessary that $\Lambda$ is a factor of an hereditary artin algebra, and if $\Lambda$ is not hereditary it is necessary that there is a simple projective $\Lambda$-module $S$ whose injective dimension is equal to gl. dim. $\Lambda$.

We end this section with some comments on the possibility of generalizing the material in this section to arbitrary artin rings (i.e. left and right artin rings). One of the essential tools used is the existence of an almost split sequence $0 \rightarrow S \rightarrow Q \rightarrow K \rightarrow 0$, with $Q$ projective, when $S$ is a simple projective noninjective $\Lambda$-module. While in general almost split sequences may not exist in $\bmod \Lambda$, we can prove that an almost split sequence of the above type exists when $S$ is simple projective and not injective. Actually we show how to construct this almost split sequence, and this at the same time gives a direct approach in this special case also for artin algebras. For $S$ simple projective we denote $S^{*} / \mathbf{r} S^{*}$ by $D^{\prime} S$. Note that if $\Lambda$ is an artin algebra, then $D^{\prime} S=$ DS.

Proposition 1.17. Let $S$ be a simple projective noninjective module over an artin ring $\Lambda$, and let $Q^{*} \stackrel{t^{*}}{\rightarrow} S^{*} \rightarrow D^{\prime} S \rightarrow 0$ be a minimal projective presentation 
in $\bmod \Lambda^{\mathrm{op}}$, where $Q$ is projective in $\bmod \Lambda$. Then we get an almost split sequence $0 \rightarrow S \stackrel{t}{\rightarrow} Q \rightarrow \operatorname{Tr} D^{\prime} S \rightarrow 0$.

Proof. The sequence $0 \rightarrow S \stackrel{t}{\rightarrow} Q \rightarrow \operatorname{Tr} D^{\prime} S \rightarrow 0$ is clearly exact since $t$ is not zero and hence a monomorphism. Also it is easy to see that it does not split. Let now $j: S \rightarrow U$ be a map which is not a split monomorphism. Assume first that $U$ is projective. Then $j^{*}: U^{*} \rightarrow S^{*}$ is not a split epimorphism. Hence $\operatorname{Im}\left(j^{*}\right) \subseteq \mathbf{r} S^{*}$, so there is a map $q^{*}: U^{*} \rightarrow Q^{*}$ such that $t^{*} q^{*}=j^{*}$, and consequently $q t=j$. If $U$ is not projective we have, since $S$ is projective, a commutative diagram

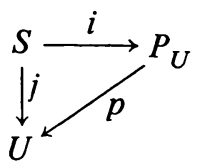

where $p: P_{U} \rightarrow U$ is a projective cover. Since $j$ is not a split monomorphism, $i$ is not, because $S$ is simple projective. By the above we then have a map $q$ : $Q \rightarrow P_{U}$ such that $q t=i$, so that $p q: Q \rightarrow U$ verifies that $(p q) t=j$. So $t$ : $S \rightarrow Q$ is left almost split. Since $\operatorname{Tr} D^{\prime} S$ is indecomposable, it follows as in the proof of Proposition 4.1 in [6], that $0 \rightarrow S \stackrel{t}{\rightarrow} Q \rightarrow \operatorname{Tr} D^{\prime} S \rightarrow 0$ is an almost split sequence.

On the basis of this proposition the Lemmas 1.1, 1.2 and 1.7 still hold if $\Lambda$ is an artin ring which is not necessarily an artin algebra. We do not know exactly how many of the other results carry over. We would also like to know if $\Gamma$ is necessarily left artin and if $(X$,$) takes \bmod \Lambda$ to $\bmod \Gamma$. If $\left(\operatorname{Tr} D^{\prime} S\right)^{*}$ $=0$, we shall see as a consequence of our results in $\$ 4$, where we consider related functors, that this turns out to be the case. Hence also Theorem 1.11 carries over to arbitrary artin rings.

2. General study of partial Coxeter functors. Let $\Lambda$ be a basic left artin ring with a simple projective noninjective module $S$, and $\Gamma$ a basic left artin ring with a simple injective nonprojective module $T$. Denote as before by $\mathcal{C}=\mathcal{C}_{s}$ the full subcategory of $\bmod \Lambda$ whose objects are the modules in $\bmod \Lambda$ having no summand isomorphic to $S$, and by $\mathscr{D}=\mathscr{D}_{T}$ the full subcategory of $\bmod \Gamma$ whose objects are the objects in $\bmod \Gamma$ with no summand isomorphic to $T$. We define a functor $F=F_{S}: \bmod \Lambda \rightarrow \bmod \Gamma$ to be a left partial Coxeter functor if $F(S)=0$ and $F$ induces an equivalence between $e_{S}$ and $\mathscr{D}_{T}$, for a simple projective noninjective $\Lambda$-module $S$ and a simple injective nonprojective $\Gamma$-module $T$. We also define a functor $G=G_{T}: \bmod \Gamma \rightarrow$ $\bmod \Lambda$ to be a right partial Coxeter functor if $G(T)=0$ and $G$ induces an equivalence of categories between $\mathscr{D}_{T}$ and $C_{S}$ for a simple injective nonprojective $\Gamma$-module $T$ and a simple projective noninjective $\Lambda$-module $S$. 
In $\$ 5$ we shall study the connection with representations of diagrams, where these concepts were originally defined. In the notation of $\$ 1$, we have seen there for an artin algebra that if $(\operatorname{Tr} D S)^{*}=0$, then $(X$,$) is a left partial$ Coxeter functor and that if $(\operatorname{Tr} T)^{*}=0$, then $Y \otimes$ is a right partial Coxeter functor. So a study of left and right partial Coxeter functors in general will give in particular more information about these functors, and this will be used in $\S 3$.

In this section we show that a left and a right partial Coxeter functor between artin rings is uniquely determined up to "equivalence" of functors in the following sense. For left artin rings $\Lambda, \Gamma, \Lambda^{\prime}, \Gamma^{\prime}$ we say that two functors $F: \bmod \Lambda \rightarrow \bmod \Gamma$ and $F^{\prime}: \bmod \Lambda^{\prime} \rightarrow \bmod \Gamma^{\prime}$ are equivalent if there are equivalences of categories $u: \bmod \Lambda \rightarrow \bmod \Lambda^{\prime}$ and $v: \bmod \Gamma \rightarrow \bmod \Gamma^{\prime}$ such that $v F=F^{\prime} u$. To prove this uniqueness we shall need some preliminary results which will be useful also in $\S 3$. We further show that up to equivalence a left partial Coxeter functor $F$ is of the following type if $\Lambda$ is $\operatorname{artin} . F=(X$,$) :$ $\bmod \Lambda \rightarrow \bmod \Gamma$, where $X=P \amalg \operatorname{Tr} D^{\prime} S, P$ is given by $S \amalg P=\Lambda, \Gamma=$ $\operatorname{End}(X)^{\text {op }}$ and $D^{\prime} S$ is, as in $\S 1$, the simple $\Lambda^{\text {op }}$-module with the same annihilator as $S$. We also show that the left partial Coxeter functors studied in $\S 1$ are essentially the only ones for artin algebras, and the same will be the case for right partial Coxeter functors. In $\$ 4$ we shall show that for an artin ring $(X$,$) as defined above is a left partial Coxeter functor when \left(\operatorname{Tr} D^{\prime} S\right)^{*}$ $=0$.

At the end of the section we get some more information about partial Coxeter functors for artin algebras, which we shall need in §3. The reason for the restriction to artin algebras is that we use the existence of almost split sequences and duality.

We start out with some preliminary results.

LEMMA 2.1. Let $\Lambda$ be a left artin ring, $\mathbb{Q}$ a full additive subcategory of $\bmod \Lambda$. Let $f: A \rightarrow B$ and $g: B \rightarrow C$ be morphisms in $\mathcal{Q}$.

(a) If $\mathcal{Q}$ is closed under kernels, then $0 \rightarrow A \stackrel{f}{\rightarrow} B \stackrel{g}{\rightarrow} C$ is exact in $\bmod \Lambda$ if and only if $0 \rightarrow(, A)|\mathbb{Q} \rightarrow(, B)| \mathbb{Q} \rightarrow(, C) \mid \mathbb{Q}$ is exact, where $(, V) \mid \mathbb{Q}$ denotes the restriction of the functor $(, V)$ to $Q$.

(b) If $\mathbb{Q}$ is closed under cokernels, then $A \stackrel{f}{\rightarrow} B \stackrel{g}{\rightarrow} C \rightarrow 0$ is exact if and only if $0 \rightarrow(C),|\Theta \rightarrow(B),| \Theta \rightarrow(A) \mid, \Theta$ is exact.

(c) If $\mathbb{Q}$ is closed under submodules or under factor modules, then $0 \rightarrow A \stackrel{f}{\rightarrow} B \stackrel{g}{\rightarrow} C \rightarrow 0$ is exact in $\bmod \Lambda$ if and only if $0 \rightarrow(, A)|\mathcal{Q} \rightarrow(, B)| \mathcal{Q}$ $\rightarrow(, C) \mid \mathbb{Q}$ and $0 \rightarrow(C),|\mathbb{Q} \rightarrow(B),| \mathbb{Q} \rightarrow(A) \mid, \mathbb{Q}$ are exact.

Proof. (a) Assume that $0 \rightarrow(, A)|\mathbb{Q} \rightarrow(, B)| \mathbb{Q} \rightarrow(, C) \mid \mathbb{Q}$ is exact. Consider the exact sequence of $\Lambda$-modules $0 \rightarrow \operatorname{Ker}(g) \rightarrow B \rightarrow C$, where by 
assumption $\operatorname{Ker}(g)$ is in $Q$. We then have the exact sequence $0 \rightarrow$ $(, \operatorname{Ker}(g))|\mathbb{Q} \rightarrow(, B)| \mathbb{Q} \rightarrow(, C) \mid \mathscr{Q}$. Since also $0 \rightarrow(, A)|\mathbb{Q} \rightarrow(, B)| \mathbb{Q} \rightarrow$ $(, C) \mid \mathcal{Q}$ is exact, we get a commutative diagram

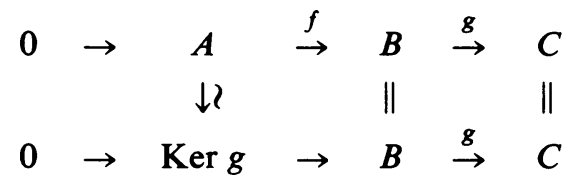

which shows that $0 \rightarrow A \stackrel{f}{\rightarrow} B \stackrel{g}{\rightarrow} C$ is exact. The other implication is trivial.

(b) The proof is similar to the proof of (a).

(c) Assume that $0 \rightarrow(, A)|\mathbb{Q} \rightarrow(, B)| \mathbb{Q} \rightarrow(, C) \mid \mathscr{Q}$ and $0 \rightarrow(C) \mid, \mathbb{Q} \rightarrow$ $(B),|\mathbb{Q} \rightarrow(A),| \mathbb{Q}$ are exact. Assume that $\mathbb{Q}$ is closed under submodules. Then it follows from (a) that $0 \rightarrow A \stackrel{f}{\rightarrow} B \stackrel{g}{\rightarrow} C$ is exact. Consider the exact sequence $0 \rightarrow A \stackrel{f}{\rightarrow} B \stackrel{g}{\rightarrow} \operatorname{Im}(g) \rightarrow 0$. Then $0 \rightarrow(\operatorname{Im}(g)),|\mathbb{Q} \rightarrow(B),| \mathbb{Q} \rightarrow$ $(A) \mid, \mathbb{Q}$ is exact. Since $\operatorname{Im}(g)$ is contained in $C, \operatorname{Im}(g)$ is in $Q$. Using that $0 \rightarrow(C),|\mathbb{Q} \rightarrow(B),| \mathbb{Q} \rightarrow(A) \mid, \mathbb{Q}$ is exact we get our desired conclusion. If $\mathbb{Q}$ is closed under factor modules, the proof is similar. The proof of the converse is trivial.

LEMMA 2.2. Let $\Lambda$ and $\Lambda^{\prime}$ be left artin rings, $\mathbb{Q}$ a full additive subcategory of $\bmod \Lambda, \mathbb{Q}^{\prime}$ a full additive subcategory of $\bmod \Lambda^{\prime}$. Assume that $\mathbb{Q}$ and $\mathbb{Q}^{\prime}$ are closed under submodules or factor modules, and that there is an equivalence of categories $\alpha: \mathbb{Q} \rightarrow \mathbb{Q}^{\prime}$.

(a) Let $f: A \rightarrow B$ and $g: B \rightarrow C$ be morphisms in $Q$. Then $0 \rightarrow A \stackrel{f}{\rightarrow} B \stackrel{g}{\rightarrow} C \rightarrow$ 0 is exact in $\bmod \Lambda$ if and only if $0 \rightarrow \alpha A \stackrel{\alpha f}{\rightarrow} \alpha B \stackrel{\alpha g}{\rightarrow} \alpha C \rightarrow 0$ is exact in $\bmod \Lambda^{\prime}$.

(b) Let $C$ in $Q$ be indecomposable.

(i) If $Q^{\prime}$ is closed under subobjects and projective covers then $\alpha(C)$ is projective if $C$ is projective.

(ii) If $\mathscr{Q}$ is closed under subobjects and projective covers, then $C$ is projective if $\alpha C$ is projective.

(c) If $Q$ is closed under extensions and all the indecomposable nonprojective objects are in $\mathcal{Q}$, then $C$ is injective if $\alpha C$ is injective.

(d) If $Q$ and $Q^{\prime}$ are closed under subobjects and projective covers, then $\alpha$ can be extended to an equivalence $\alpha_{1}: \mathbb{Q}_{1} \rightarrow \mathbb{Q}_{1}^{\prime}$. Here the objects of $\mathbb{Q}_{1}$ are the $M$ in $\bmod \Lambda$ such that there is an exact sequence $P_{1} \rightarrow P_{0} \rightarrow M \rightarrow 0$ with $P_{1}, P_{0}$ projective and in $Q$, and the objects in $\mathbb{Q}_{1}^{\prime}$ are defined analogously.

Proof. (a) is an immediate consequence of Lemma 2.1.

(b)(i) Assume that $C$ is indecomposable projective and in $\mathscr{Q}$. Since $\mathbb{Q}^{\prime}$ is closed under subobjects and projective covers, we have an exact sequence $0 \rightarrow \alpha A \rightarrow \alpha B \rightarrow \alpha C \rightarrow 0$, where $\alpha B$ is projective in mod $\Lambda^{\prime}$. By (a) we then have an exact sequence $0 \rightarrow A \rightarrow B \rightarrow C \rightarrow 0$, which must split since $C$ is 
projective. Hence $\alpha B \rightarrow \alpha C$ is a split epimorphism, which shows that $\alpha C$ is projective.

(ii) The proof is analogous to the proof of (i).

(c) Let $C$ be in $Q$ such that $\alpha C$ is injective in $\bmod \Lambda^{\prime}$. Suppose $K$ is indecomposable in $Q$ and consider an exact sequence $0 \rightarrow C \rightarrow E \rightarrow K \rightarrow 0$. By assumption $E$ is in $Q$, so that by (a) $0 \rightarrow \alpha C \rightarrow \alpha E \rightarrow \alpha K \rightarrow 0$ is exact. This splits if $C$ is injective, hence $0 \rightarrow C \rightarrow E \rightarrow K \rightarrow 0$ splits. This shows that $\operatorname{Ext}_{\Lambda}^{1}(K, C)=0$. Since every indecomposable object in $\bmod \Lambda$ not in $Q$ is projective, we conclude that $\operatorname{Ext}_{\Lambda}^{1}(, C)$ is zero, which implies that $C$ is injective.

(d) Assume that $Q$ and $\mathcal{Q}^{\prime}$ are closed under subobjects and projective covers. By (b) there is induced an equivalence between the full subcategory of projective objects in $\mathbb{Q}$ and in $\mathbb{Q}^{\prime}$, which induces an equivalence $\alpha_{1}: \mathbb{Q}_{1} \rightarrow \mathbb{Q}_{1}^{\prime}$. Here $\alpha_{1}$ is defined in the following way. If $M$ is indecomposable and in $\mathbb{Q}_{1}$ we have by assumption an exact sequence $P_{1} \stackrel{f}{\rightarrow} P_{0} \rightarrow M \rightarrow 0$ with $P_{1}, P_{0}$ projective and in $\mathcal{Q}$. We then define $\alpha_{1}(M)=$ Coker $\alpha(f)$. We now want to show that $\alpha_{1}$ extends $\alpha$. Let $C$ be indecomposable in $\mathcal{Q}$, and $P_{1} \stackrel{f}{\rightarrow} P_{0} \stackrel{g}{\rightarrow} C \rightarrow 0$ a minimal projective presentation. By the assumption on $\mathbb{Q}$ we know that $P_{1}$ and $P_{0}$ are in $Q$. It is then not hard to see that $\alpha P_{1} \rightarrow \alpha P_{0} \rightarrow \alpha C \rightarrow 0$ is exact, and this finishes the proof.

We are now ready to deduce some properties about partial Coxeter functors, in particular, to get uniqueness and a description of what they look like up to equivalence.

We first recall that if $t: \Lambda_{1} \rightarrow \Lambda_{2}$ is a ring isomorphism, then $t$ induces naturally an equivalence of categories $t^{*}: \bmod \Lambda_{2} \rightarrow \bmod \Lambda_{1}$ in the following way. For a $\Lambda_{2}$-module $M, t^{*}(M)$ is the abelian group $M$ with the $\Lambda_{1}$-module structure induced by $t$. That is, $\lambda_{1} m=t\left(\lambda_{1}\right) m$, for $\Lambda_{1}$ in $\Lambda_{1}, m$ in $M$.

TheOREM 2.3. Let $\Lambda$ and $\Gamma$ be left artin rings, $S$ a simple projective noninjective $\Lambda$-module, $F=F_{S}: \bmod \Lambda \rightarrow \bmod \Gamma$ a left partial Coxeter functor, and let $T$ denote the simple injective nonprojective $\Gamma$-module associated to $F$.

(a) $F$ is left exact and if $0 \rightarrow A \rightarrow B \rightarrow C \rightarrow 0$ is exact with $A$ in $\mathcal{C}=\mathcal{C}_{s}$, then $0 \rightarrow F(A) \rightarrow F(B) \rightarrow F(C) \rightarrow 0$ is exact.

(b) If $F^{\prime}=F_{S}^{\prime}: \bmod \Lambda \rightarrow \bmod \Gamma^{\prime}$ is a left partial Coxeter functor, then there is a ring isomorphism $t: \Gamma^{\prime} \rightarrow \Gamma$ such that we have a commutative diagram

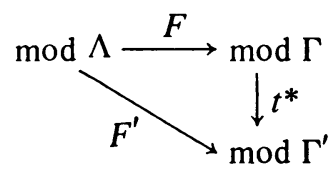

where $t^{*}$ is an equivalence of categories induced by $t: \Gamma^{\prime} \rightarrow \Gamma$.

(c) $F$ has a left adjoint $G$ which is a right partial Coxeter functor. 
(d) If $\Lambda$ is artin, then $F$ is equivalent to $(X):, \bmod \Lambda \rightarrow \bmod \operatorname{End}(X)^{\text {op }}$, where $P$ is given by $\Lambda=S \amalg P, X=P \amalg \operatorname{Tr} D^{\prime} S$, and $\left(\operatorname{Tr} D^{\prime} S\right)^{*}=0$.

(e) Assume that the indecomposable injective modules over $\Lambda$ and $\Gamma$ are finitely generated. Let I denote the direct sum of one copy of each of the indecomposable injective $\Lambda$-modules and $E$ the direct sum of one copy of each of the indecomposable injective $\Gamma$-modules. Let $I_{1}$ be an injective envelope of $S$, $I=I_{1} \amalg I_{2}, E=T \amalg E_{1}$. Then $F(I)=F\left(I_{1}\right) \amalg E_{1}$ and $E=T \amalg F\left(I_{2}\right)$.

Proof. (a) Let $0 \rightarrow A \stackrel{f}{\rightarrow} B \stackrel{g}{\rightarrow} C \rightarrow 0$ be an exact sequence in $\bmod \Lambda$. We

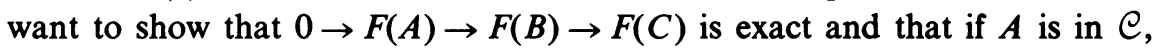
then $0 \rightarrow F(A) \rightarrow F(B) \rightarrow F(C) \rightarrow 0$ is exact. We can clearly assume that the sequence has no split exact summands. Then it is not hard to see that $B$ and $C$ must be in $C$. Since $C$ is clearly closed under factor modules and $\mathscr{D}=\mathscr{D}_{T}$ is closed under submodules, we conclude by Lemma 2.2 that if $A$ is in $\mathrm{C}$, then $0 \rightarrow F(A) \rightarrow F(B) \rightarrow F(C) \rightarrow 0$ is exact. If $A$ is not in $\mathcal{C}$ we write $A=A_{1} \amalg S^{t}$ for some $t>0$. Since $S$ is simple projective, $(, S) \mid \mathcal{C}$ is zero, so we get the exact sequence $0 \rightarrow\left(, A_{1}\right)|\mathcal{C} \rightarrow(, B)| \mathcal{C} \rightarrow(, C) \mid \mathcal{C}$. Hence we get an exact sequence $0 \rightarrow\left(, F\left(A_{1}\right)\right)|\mathscr{D} \rightarrow(, F(B))| \mathscr{D}_{\rightarrow} \rightarrow(, F(C)) \mid \mathscr{D}_{\text {. Since }} \mathscr{D}_{T}$ is closed under submodules, it follows from Lemma 2.1 that $0 \rightarrow F\left(A_{1}\right) \rightarrow F(B) \rightarrow$ $F(C)$ is exact. Since $F(S)=0$ we then have that $0 \rightarrow F(A) \rightarrow F(B) \rightarrow F(C)$ is exact.

(b) Let $F^{\prime}=F_{S}^{\prime}: \bmod \Lambda \rightarrow \bmod \Gamma^{\prime}$ be a left partial Coxeter functor. We want to show that there is a close connection between $F^{\prime}$ and the left partial Coxeter functor $F: \bmod \Lambda \rightarrow \bmod \Gamma$.

Denote by $T^{\prime}$ the simple injective nonprojective $\Gamma^{\prime}$-module such that the left partial Coxeter $F^{\prime}$ induces an equivalence between $C_{S}$ and $\mathscr{D}_{T^{\prime}}$. We have a commutative diagram

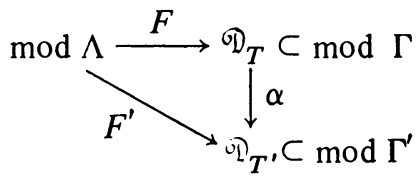

where $\alpha$ is an equivalence of categories. Since $\mathscr{D}_{T}$ and $\mathscr{D}_{T^{\prime}}$ are closed under submodules and contain all finitely generated projective modules, it follows from Lemma 2.2 that $\alpha$ can be extended to an equivalence $t^{*}: \bmod \Gamma \rightarrow$ mod $\Gamma^{\prime}$. Since the rings are basic, $t^{*}$ is induced by a ring isomorphism $t$ : $\Gamma^{\prime} \rightarrow \Gamma$.

(c) We want to show that the left partial Coxeter functor $F$ has a left adjoint $G$ which is a right partial Coxeter functor. Let $\beta$ : $\mathscr{D}_{T} \rightarrow \mathcal{C}_{S}$ be an inverse equivalence, and define $G$ as an extension of $\beta$, by defining $G(T)=0$. It is then easy to see that $F$ and $G$ are adjoint functors. 
(d) We want to show that the left partial Coxeter functor $F$ is equivalent to $(X):, \bmod \Lambda \rightarrow \bmod$ End $(X)^{\text {op }} . F$ is isomorphic to $(G(\Gamma)):, \bmod \Lambda \rightarrow$ $\bmod \Gamma$, where $G(\Gamma)$ is a $\lambda$ - $\Gamma$-bimodule in a natural way. Since $\Gamma \rightarrow \operatorname{End}(\Gamma)^{\text {op }}$ $\rightarrow$ End $G(\Gamma)^{\text {op }}$ is a ring isomorphism, we have a commutative diagram

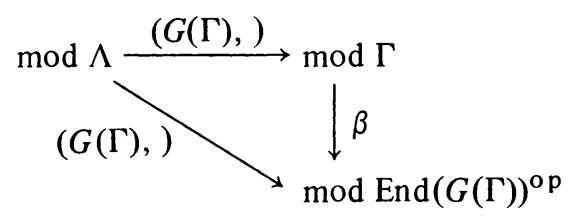

where $\beta$ is an equivalence of categories. Hence $F$ is equivalent to $(G(\Gamma)$, ): $\bmod \Lambda \rightarrow \bmod \operatorname{End}(G(\Gamma))^{\text {op }}$.

To get our desired result we want to show that $G(\Gamma)$ is isomorphic to $X$. To compute $G(\Gamma)$ we want to find the indecomposable $C$ in $\bmod \Lambda$ such that $F(C)$ is projective. Let $Q$ be indecomposable projective not isomorphic to $S$. Then it follows from Lemma $2.2(\mathrm{~b})$ that $F(Q)$ is projective.

Write $\Lambda=P \amalg S$. Then we can write $G(\Gamma)=P \amalg Y$. If $Y=0, G(\Gamma)$ would be projective, and it is then not hard to see that every object in mod End $G(\Gamma)^{\text {op }}$ would be isomorphic to an object of the form $(G(\Gamma), U)$, for $U$ in $\bmod \Lambda$. We want to show that $Y=\operatorname{Tr} D^{\prime} S$. Let $Y_{1}$ be an indecomposable summand of $Y$. Since $\left(Y_{1}, S\right)$ is zero, $Y_{1}$ is not isomorphic to $S$, and consequently $Y_{1}$ is not projective. We claim that $\operatorname{Ext}^{1}\left(Y_{1}, A\right)=0$ for each indecomposable $A$ in $\mathcal{C}$. For otherwise we would have a nonsplit exact sequence $0 \rightarrow A \rightarrow E \rightarrow Y_{1} \rightarrow 0$. But since, by $(\mathrm{a}), 0 \rightarrow\left(Y_{1}, A\right) \rightarrow\left(Y_{1}, E\right) \rightarrow$ $\left(Y_{1}, Y_{1}\right) \rightarrow 0$ would be exact, we would get a contradiction. Since $Y_{1}$ is not projective, we must then have $\operatorname{Ext}^{1}\left(Y_{1}, S\right) \neq 0$. Consider now the almost split sequence $0 \rightarrow S \rightarrow Q \rightarrow \operatorname{Tr} D^{\prime} S \rightarrow 0$ (see Proposition 1.17). This gives rise to the exact sequence $0 \rightarrow\left(Y_{1}, S\right) \rightarrow\left(Y_{1}, Q\right) \rightarrow\left(Y_{1}, \operatorname{Tr} D^{\prime} S\right) \rightarrow \operatorname{Ext}^{1}\left(Y_{1}, S\right) \rightarrow$ 0 . There is then a map $h: Y_{1} \rightarrow \operatorname{Tr} D^{\prime} S$ which does not factor through $Q$. Since $0 \rightarrow S \rightarrow Q \rightarrow \operatorname{Tr} D^{\prime} S \rightarrow 0$ is almost split and $Y_{1}$ is indecomposable, $h$ : $Y_{1} \rightarrow \operatorname{Tr} D^{\prime} S$ must be an isomorphism. This shows that $G(\Gamma)=P \amalg \operatorname{Tr} D^{\prime} S$ $=X$. To finish the proof of (d) it only remains to show that $\left(\operatorname{Tr} D^{\prime} S\right)^{*}$ is zero.

To see this, consider again the almost split sequence $0 \rightarrow S \rightarrow Q \rightarrow \operatorname{Tr} D^{\prime} S$ $\rightarrow 0$, and the minimal projective resolution $0 \rightarrow(X, Q) \rightarrow\left(X, \operatorname{Tr} D^{\prime} S\right) \rightarrow T_{1}$ $\rightarrow 0$ in $\bmod \Gamma$. We want to show that $T_{1}$ is isomorphic to the simple injective $\Gamma$-module $T$. We know that $T_{1}=\operatorname{Ext}_{\Lambda}^{1}\left(\operatorname{Tr} D^{\prime} S, S\right)$. To show that $T_{1}$ is simple, we first show that any nonzero element in $\operatorname{Ext}_{\Lambda}^{1}\left(\operatorname{Tr} D^{\prime} S, S\right)$ is an almost split sequence. To see this, let $0 \rightarrow S \rightarrow E \rightarrow \operatorname{Tr} D^{\prime} S \rightarrow 0$ be a nonsplit exact sequence, and consider the commutative diagram 


$$
\begin{aligned}
& 0 \rightarrow \underset{\downarrow t}{S} \rightarrow \underset{\downarrow s}{E} \rightarrow \underset{\downarrow \|}{\operatorname{Tr} D^{\prime} S} \rightarrow 0 \\
& 0 \rightarrow S \rightarrow Q \rightarrow \operatorname{Tr} D^{\prime} S \rightarrow 0
\end{aligned}
$$

where $t$ must clearly be an isomorphism, and hence also $s$. Using this it is not hard to see that $T_{1}=\operatorname{Ext}_{\Lambda}^{1}\left(\operatorname{Tr} D^{\prime} S, S\right)$ is a simple $\Gamma$-module. To show that $T_{1}$ is isomorphic to $T$ it is then sufficient to show that $\left(X, \operatorname{Tr} D^{\prime} S\right)$ is a projective cover for $T$. Assume to the contrary that $\left(X, P_{1}\right) \rightarrow T$ is a projective cover, where $P_{1}$ is an indecomposable projective $\Lambda$-module. Since every submodule of $\left(X, P_{1}\right)$ is in $\mathscr{D}_{T}$, we have an exact sequence $0 \rightarrow(X, K) \stackrel{u}{\rightarrow}\left(X, P_{1}\right) \rightarrow T \rightarrow$ 0 , with $K$ in $\bmod \Lambda$. Since $F \mid \mathcal{C}$ is fully faithful, $u=\left(X, u_{1}\right)$, for some $u_{1}$ : $K \rightarrow P_{1}$. Consider the exact sequence $K \rightarrow P_{1} \rightarrow C \rightarrow 0$. We then get an exact sequence $0 \rightarrow\left(\left(X, P_{1}\right),\right)|\mathscr{D} \rightarrow((X, K)),| \mathscr{D}$, hence an exact sequence $0 \rightarrow$ $\left(P_{1},\right)|\mathcal{C} \rightarrow(K),| \mathcal{C}$. Since we also have the exact sequence $0 \rightarrow(C) \mid, \mathcal{C} \rightarrow$ $(P),|\mathcal{C} \rightarrow(K),| \mathcal{C}$ and $C$ is obviously in $\mathcal{C}$, we conclude that $C$ is zero, so that $K \rightarrow P$ is an epimorphism. Hence we have our desired contradiction, so that $T=T^{\prime}$. Since $T$ is simple injective, $T$ cannot occur in the composition series of any indecomposable projective $\left(X, P_{1}\right)$, where $P_{1}$ is an indecomposable projective $\Lambda$-module. This implies that $\operatorname{Hom}\left(\left(X, \operatorname{Tr} D^{\prime} S\right),(X, P)\right)$ is zero, and consequently $\left(\operatorname{Tr} D^{\prime} S\right)^{*}$ is zero. This finishes the proof of (d).

(e) Assume that the indecomposable injective modules over $\Lambda$ and $\Gamma$ are finitely generated. We first want to show that if $I_{1}$ is an injective envelope for $S$, then $F\left(I_{1}\right)$ is not injective. Consider the exact sequence $0 \rightarrow S \rightarrow I_{1} \rightarrow I_{1} / S$ $\rightarrow 0$. Since $F$ is left exact, we get the exact sequence $0 \rightarrow F\left(I_{1}\right) \rightarrow F\left(I_{1} / S\right)$. This does not split since $I_{1} \rightarrow I_{1} / S$ is not a split monomorphism. Hence $F\left(I_{1}\right)$ is not injective. If $C$ is indecomposable and $F(C)$ is injective, we know by Lemma 2.2(c) that $C$ is injective. Let now $I_{2}$ denote the direct sum of the indecomposable injective $\Lambda$-modules not isomorphic to $I_{1}$. By (c) we know that $\Lambda$ and $\Gamma$ have the same number of indecomposable projective modules. Hence a counting argument implies that $F\left(I_{2}\right)$ is injective. The rest of (e) is now easy.

We get the following analogue for right partial Coxeter functors.

THeOREM 2.4. Let $\Gamma$ and $\Lambda$ be left artin rings, $T$ a simple injective nonprojective $\Gamma$-module, $G=G_{T}: \bmod \Gamma \rightarrow \bmod \Lambda$ a right partial Coxeter functor, and $S$ the associated simple projective noninjective $\Lambda$-module.

(a) $G$ is right exact and if $0 \rightarrow A \rightarrow B \rightarrow C \rightarrow 0$ is exact in mod $\Gamma$ with $C$ in $\mathscr{D}=\mathscr{D}_{T}$, then $0 \rightarrow G(A) \rightarrow G(B) \rightarrow G(C) \rightarrow 0$ is exact.

(b) If $G^{\prime}=G_{T}^{\prime}: \bmod \Gamma \rightarrow \bmod \Lambda^{\prime}$ is a right partial Coxeter functor and $\Lambda$ and $\Lambda^{\prime}$ are artin, then there is a ring isomorphism $t: \Lambda^{\prime} \rightarrow \Lambda$ and a commutative diagram 


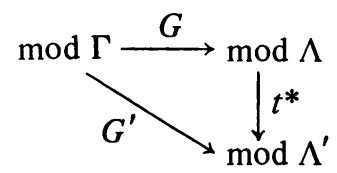

where $t^{*}$ is an equivalence of categories induced by $t: \Lambda^{\prime} \rightarrow \Lambda$.

(c) $G$ has as an adjoint a left partial Coxeter functor $F$.

Proof. (a) and (c) follow as before.

(b) We have a commutative diagram

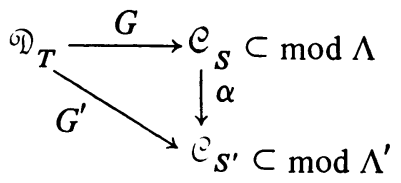

where $\alpha$ is an equivalence of categories. Let $0 \rightarrow S \rightarrow Q \stackrel{g}{\rightarrow} \operatorname{Tr} D^{\prime} S \rightarrow 0$ be an almost split sequence. Then we have seen that $0 \rightarrow F(Q) \rightarrow F\left(\operatorname{Tr} D^{\prime} S\right) \rightarrow T$ $\rightarrow 0$ is a minimal projective presentation in $\bmod \Gamma$, where $F$ denotes an inverse equivalence for $G$. Using the converse result, we then get an almost split sequence $0 \rightarrow S^{\prime} \rightarrow \alpha(Q) \stackrel{\alpha(g)}{\rightarrow} \alpha\left(\operatorname{Tr} D^{\prime} S\right) \rightarrow 0$ in mod $\Lambda^{\prime}$. Since $0 \rightarrow S \rightarrow$ $Q \rightarrow \operatorname{Tr} D^{\prime} S \rightarrow 0$ is almost split, we have for all $K$ in $\bigodot_{S}$ an exact sequence $0 \rightarrow\left(\operatorname{Tr} D^{\prime} S, K\right) \rightarrow(Q, K) \rightarrow(S, K) \rightarrow 0$. Hence $0 \rightarrow\left(\alpha\left(\operatorname{Tr} D^{\prime} S\right), \alpha(K)\right) \rightarrow$ $(\alpha(Q), \alpha(K)) \rightarrow\left(S^{\prime}, \alpha(K)\right) \rightarrow 0$ is also exact. This gives a natural isomorphism $(S, K) \rightarrow\left(S^{\prime}, \alpha(K)\right)$. To define a map $(S, S) \rightarrow\left(S^{\prime}, S^{\prime}\right)$ consider for $u$ : $S \rightarrow S$ the diagram

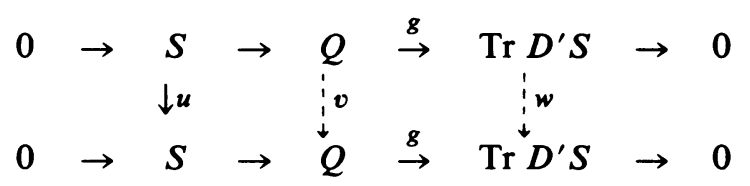

and the induced diagram:

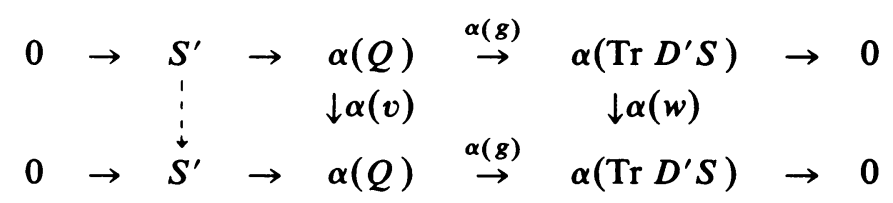

We can now extend $\alpha$ to an equivalence $t^{*}: \bmod \Lambda \rightarrow \bmod \Lambda^{\prime}$, by defining $t^{*}(S)=S^{\prime}$ and using the natural isomorphisms $(S, K) \rightarrow\left(S^{\prime}, \alpha(K)\right)$ and $(S, S) \rightarrow\left(S^{\prime}, S^{\prime}\right)$ described above. Since the rings are basic, $t^{*}$ is induced by a ring isomorphism $t: \Lambda^{\prime} \rightarrow \Lambda$.

We shall now get some additional information about partial Coxeter functors in the case of artin algebras, which will be useful in \$3. Actually, for parts (a) and (b) it will be sufficient to assume that our artin rings have a 
duality and that almost split sequences exist in $\bmod \Lambda$ and $\bmod \Gamma . D \operatorname{Tr}$ would then be replaced by a map $\gamma$ from the indecomposable nonprojective objects to the indecomposable noninjective objects in $\bmod \Lambda$, given by the almost split sequence $0 \rightarrow \gamma(C) \rightarrow B \rightarrow C \rightarrow 0$.

Proposition 2.5. Let $F: \bmod \Lambda \rightarrow \bmod \Gamma$ be a left partial Coxeter functor, where $\Lambda$ and $\Gamma$ are artin algebras. Let $S$ be the associated simple projective $\Lambda$-module and $T$ the associated simple injective $\Gamma$-module. Then we have the following.

(a) If $A$ is an indecomposable noninjective $\Lambda$-module not isomorphic to $S$ and $0 \rightarrow A \rightarrow B \rightarrow C \rightarrow 0$ is an almost split sequence, then $0 \rightarrow F(A) \rightarrow F(B) \rightarrow$ $F(C) \rightarrow 0$ is also an almost split sequence, $F(\operatorname{Tr} D A) \simeq \operatorname{Tr} D F(A)$, and $F(D \operatorname{Tr} C) \cong D \operatorname{Tr} F(C)$.

(b) $F\left(I_{1}\right) \cong D \operatorname{Tr} T$, where $I_{1}$ is an injective envelope for $S$.

(c) $\Lambda \cong \operatorname{End}_{\Gamma}(Y)$, where $Y=\operatorname{Tr} T \amalg D(I)$, and I is the sum of one copy of each of the indecomposable injective $\Gamma$-modules not isomorphic to $T$.

Proof. Let $A$ be an indecomposable $\Lambda$-module which is not injective and not isomorphic to $S$, and let $0 \rightarrow A \stackrel{f}{\rightarrow} B \stackrel{g}{\rightarrow} C \rightarrow 0$ be an almost split sequence. Since the sequence has no split exact summands, we know that $B$ and $C$ are in $\mathcal{C}$, so that $0 \rightarrow F(A) \stackrel{F(f)}{\rightarrow} F(B) \stackrel{F(g)}{\rightarrow} F(C) \rightarrow 0$ is exact by Theorem 2.3(a). It does not split since $0 \rightarrow A \stackrel{f}{\rightarrow} B \stackrel{g}{\rightarrow} C \rightarrow 0$ does not split.

Let $h: F(A) \rightarrow M$ be a map which is not a splittable monomorphism. If $M$ is isomorphic to the simple injective $\Gamma$-module $T$ then there is a map $g$ : $F(B) \rightarrow M$ such that $g f=h$. If $M$ is indecomposable and not isomorphic to $T$ then $M=F(N)$ for some $\Lambda$-module $N$. Since $F \mid \mathcal{C}: \mathcal{C} \rightarrow \mathscr{D}$ is an equivalence of categories, there is a map $h_{1}: A \rightarrow N$ such that $F\left(h_{1}\right)=h$. Then $h_{1}$ is not a splittable monomorphism, because $h$ is not a splittable monomorphism. The sequence $0 \rightarrow A \stackrel{f}{\rightarrow} B \stackrel{g}{\rightarrow} C \rightarrow 0$ is almost split, so there is some $v: B \rightarrow N$ such that $v f=h_{1}$. Then $F(v) F(f)=F\left(h_{1}\right)=h$, and therefore the sequence $0 \rightarrow$ $F(A) \stackrel{F(f)}{\rightarrow} F(B) \stackrel{F(g)}{\rightarrow} F(C) \rightarrow 0$ is almost split. The rest of (a) follows from the above and the fact that since $0 \rightarrow F(A) \rightarrow F(B) \rightarrow F(C) \rightarrow 0$ and $0 \rightarrow A \rightarrow B$ $\rightarrow C \rightarrow 0$ are almost split, then $A \cong D \operatorname{Tr} C$ and $F(A) \cong D \operatorname{Tr} F(C)$.

(b) $D \operatorname{Tr} T$ is not injective and is, therefore, in $\mathscr{D}$. So there is some $E$ in $\mathcal{C}$ such that $D \operatorname{Tr} T \cong F(E)$. If $E$ is not injective then we know by (a) that $F(\operatorname{Tr} D E) \cong \operatorname{Tr} D F(E)$. Hence we get $T \cong F(\operatorname{Tr} D E)$, and this is a contradiction. So $E$ is injective and, since $D \operatorname{Tr} T \cong F(E)$ is not injective, it follows from Theorem 2.3(b) that $E=I_{1}$ is the injective envelope of $S$.

(c) Let $\left\{I_{1}, \ldots, I_{n}\right\}$ be a complete set of nonisomorphic injective $\Lambda$-modules. We know by Theorem 2.3(e) that the sum of one copy of each of the indecomposable injective $\Gamma$-modules not isomorphic to $T$ is isomorphic to 
$F\left(I_{2}\right) \amalg \cdots \amalg F\left(I_{n}\right)$. And by (b) we know that $F\left(I_{1}\right) \cong D \operatorname{Tr} T$. Therefore for $Y=\operatorname{Tr} T \amalg D(I)$, where $I$ is the sum of one copy of each of the indecomposable injective $\Gamma$-modules not isomorphic to $T, D(Y)=D \operatorname{Tr} T \amalg I=$ $F\left(I_{1}\right) \amalg F\left(I_{2}\right) \amalg \cdots \amalg F\left(I_{n}\right)=F(D(\Lambda))$. Then $\operatorname{End}_{\Gamma}(Y) \cong \operatorname{End}_{\Gamma}(D Y)^{\mathrm{op}} \cong$ $\operatorname{End}_{\Gamma}(F(D \Lambda))^{\text {op }} \cong \operatorname{End}_{\Lambda}(D \Lambda)^{\text {op }} \cong\left(\operatorname{End}_{\Lambda}\left(\Lambda_{\Lambda}\right)\right) \cong \Lambda$.

For right partial Coxeter functors we have the following result which is analogous to Proposition 2.5.

Proposition 2.6. Let $\Lambda$ and $\Gamma$ be artin algebras. Let $G: \bmod \Gamma \rightarrow \bmod \Lambda$ be a right partial Coxeter functor. Then we have the following.

(a) If $C$ is an indecomposable nonprojective $\Gamma$-module not isomorphic to $T$ and the sequence $0 \rightarrow A \rightarrow B \rightarrow C \rightarrow 0$ is almost split, then $0 \rightarrow G(A) \rightarrow G(B) \rightarrow$ $G(C) \rightarrow 0$ is also an almost split sequence. Therefore $D \operatorname{Tr} G(C) \cong G(D \operatorname{Tr} C)$ and $\operatorname{Tr} D G(A) \cong G(\operatorname{Tr} D A)$.

(b) $G(D \operatorname{Tr} T)$ is an injective $\Lambda$-module. If $\left\{T, I_{2}, \ldots, I_{n}\right\}$ is a complete set of nonisomorphic indecomposable injective $\Gamma$-modules, then $\left\{G(D \operatorname{Tr} T), G\left(I_{2}\right), \ldots, G\left(I_{n}\right)\right\}$ is a complete set of nonisomorphic indecomposable injective $\Lambda$-modules.

(c) $\Lambda \cong \operatorname{End}_{\Gamma}(Y)$, where $Y=\operatorname{Tr} T \amalg D I$, and $I$ is the sum of one copy of each of the indecomposable injective $\Gamma$-modules not isomorphic to $T$.

We now apply some of the above results to obtain more information about the partial Coxeter functors $F$ and $G$ studied in $\$ 1$.

Proposition 2.7. Let $\Lambda$ be an artin algebra with a simple projective noninjective module $S$ and assume that $(\operatorname{Tr} D S)^{*}=0$. Let $\Gamma=\operatorname{End}_{\Lambda}(X)^{\mathrm{op}}$, where $X=P \amalg T r D S$ and $P$ is determined by $\Lambda=P \amalg S$. Let $I$ be the sum of one copy of each of the indecomposable injective $\Gamma$-modules not isomorphic to $T=\operatorname{Ext}_{\Lambda}^{1}(X, S)$, and let $Y=\operatorname{Tr} T \amalg D I$. Then we have the following.

(a) ${ }_{\Lambda} X_{\Gamma}$ and ${ }_{\Lambda} Y_{\Gamma}$ are isomorphic $\Lambda$ - $\Gamma$-bimodules, where $X$ and $Y$ are considered as modules over their endomorphism rings in the usual way.

(b) The functors $F=\operatorname{Hom}_{\Lambda}(X):, \bmod \Lambda \rightarrow \bmod \Gamma$ and $G=Y \otimes: \bmod \Gamma$ $\rightarrow \bmod \Lambda$ are adjoint functors.

(c) Let $\mathcal{C}$ and $\mathscr{D}$ be the categories considered at the beginning of the section and let $F$ and $G$ be as in $(b)$. Then $F \mid \mathcal{C}: \mathcal{C} \rightarrow \mathscr{D}$ and $G \mid \mathscr{D}: \mathscr{D} \rightarrow \mathcal{C}$ are inverse equivalences of categories.

Proof. (a) We have seen that $F$ is a left partial Coxeter functor, so applying Proposition 2.5 we have that $F(D \Lambda) \cong D \operatorname{Tr} T \amalg I$. Then $Y=$ $\operatorname{Tr} T \amalg D I=D F(D \Lambda)=D \operatorname{Hom}_{\Lambda}(X, D \Lambda)$. Since there is an isomorphism $X \rightarrow D \operatorname{Hom}_{\Lambda}(X, D \Lambda)$ functorial in $X$ (see [11, p. 119]), $X$ and $Y$ are isomorphic $\Lambda$ - $\Gamma$-bimodules.

(b) is an immediate consequence of (a). 
(c) follows from the fact that an inverse equivalence of $F: \mathcal{C} \rightarrow \mathbb{D}$ extends to a right partial Coxeter functor which is adjoint to $F$.

3. Hereditary artin algebras. In this section we study hereditary basic artin algebras. If $S$ is a simple projective noninjective module over an hereditary artin algebra $\Lambda$, then we have $(\operatorname{Tr} D S)^{*}=0$, so that any functor of the type $(X):, \bmod \Lambda \rightarrow \bmod \Gamma$, where $X=P \amalg T r D S$ and as usual $\Lambda=P \amalg S$, is a partial Coxeter functor. In the introduction we pointed out that for an hereditary artin algebra $\Lambda, D \operatorname{Tr}$ is a functor from $\bmod \Lambda$ to $\bmod \Lambda$, taking projectives to zero and inducing an equivalence of categories between $\bmod _{P} \Lambda$ and $\bmod _{I} \Lambda$. Our main result in this section is that $D \operatorname{Tr}$ can be written as a composition of partial Coxeter functors in a natural way.

We recall that all the artin algebras that we consider are basic. Throughout this section $\Lambda$ will denote an indecomposable hereditary basic artin algebra which is not simple.

Since $\Lambda$ is hereditary we can define a partial order in the set of indecomposable projective $\Lambda$-modules by writing $P \leqslant Q$ if and only if $\operatorname{Hom}_{\Lambda}(P, Q) \neq$ 0 . In analogy with admissible sequences of simple modules (see $[3, \S 3])$ we say that $P_{1}, \ldots, P_{n}$ is an admissible sequence of projective $\Lambda$-modules if $P_{1}, \ldots, P_{n}$ is a complete set of nonisomorphic indecomposable projective $\Lambda$-modules such that $i<j$ implies $P_{j} \nless P_{i}$, that is, $\operatorname{Hom}_{\Lambda}\left(P_{j}, P_{i}\right)=0$. When $\Lambda$ is hereditary admissible sequences always exist. For example if $P_{1}, \ldots, P_{n}$ are the nonisomorphic indecomposable projective $\Lambda$-modules and length $P_{i}<$ length $P_{j}$ for $i<j$, then the sequence $P_{1}, \ldots, P_{n}$ is admissible.

We shall denote a pair consisting of an hereditary artin algebra $\Lambda$ and an admissible sequence $P_{1}, \ldots, P_{n}$ of projective $\Lambda$-modules by $\left(\Lambda ; P_{1}, \ldots, P_{n}\right)$. We now describe a particular way of associating with a pair $\left(\Lambda ; P_{1}, \ldots, P_{n}\right)$ another pair $\left(\Lambda_{1} ; P_{1}^{\prime}, \ldots, P_{n}^{\prime}\right)$, where $\Lambda_{1}$ is an hereditary artin algebra and $P_{1}^{\prime}, \ldots, P_{n}^{\prime}$ is an admissible sequence of projective $\Lambda_{1}$-modules.

The projective $\Lambda$-module $P_{1}$ is simple, because $\left(P_{i}, P_{1}\right)=0$ for $i>1$ and is, therefore, not injective since we have assumed that $\Lambda$ is indecomposable. Let $P=P_{2} \amalg \cdots \amalg P_{n}$, let $X=P \amalg \operatorname{Tr} D P_{1}$ and let $\Lambda_{1}^{\prime}=\operatorname{End}_{\Lambda}(X)^{\mathrm{op}}$. Then, by Proposition 1.13, $\Lambda_{1}$ is an hereditary artin algebra. Let $F$ denote the partial Coxeter functor $(X):, \bmod \Lambda \rightarrow \bmod \Lambda_{1}$. Then, because $F$ restricted to the full subcategory of mod $\Lambda$ whose objects do not contain a summand isomorphic to $P_{1}=S$ is fully faithful (Proposition 1.4) and $(\operatorname{Tr} D S)^{*}=0$, we know that the sequence $P_{1}^{\prime}, \ldots, P_{n}^{\prime}$ of projective $\Lambda_{1}$-modules, where $P_{1}^{\prime}=F\left(P_{2}\right)$, $P_{2}^{\prime}=F\left(P_{3}\right), \ldots, P_{n-1}^{\prime}=F\left(P_{n}\right), P_{n}^{\prime}=F\left(\operatorname{Tr} D P_{1}\right)$, is an admissible sequence of nonisomorphic projective modules. Thus associated with the pair $\left(\Lambda ; P_{1}, \ldots, P_{n}\right)$ there is the pair $\left(\Lambda_{1}, P_{1}^{\prime}, \ldots, P_{n}^{\prime}\right)$.

Iterating this process we obtain from the pair $\left(\Lambda ; P_{1}, \ldots, P_{n}\right)$ a sequence of pairs $\left(\Lambda_{0} ; P_{1}^{(0)}, \ldots, P_{n}^{(0)}\right),\left(\Lambda_{1}, P_{1}^{(1)}, \ldots, P_{n}^{(1)}\right), \ldots,\left(\Lambda_{n} ; P_{1}^{(n)}, \ldots, P_{n}^{(n)}\right)$, 
where $\left(\Lambda_{0} ; P_{1}^{(0)}, \ldots, P_{n}^{(0)}\right)=\left(\Lambda ; P_{1}, \ldots, P_{n}\right)$. We denote by $F_{i}$ the partial Coxeter functor

$$
\left(X_{i},\right): \bmod \Lambda_{i} \rightarrow \bmod \Lambda_{i+1},
$$

where $X_{i}=P_{2}^{(i)} \amalg \cdots \amalg P_{n}^{(i)} \amalg \operatorname{Tr} D P_{1}^{(i)}$.

Before we state the main result of this section we need some preliminary results.

LeMMA 3.1. Given $\left(\Lambda ; P_{1}, \ldots, P_{n}\right)$ and the pair $\left(\Lambda_{i} ; P_{1}^{(i)}, \ldots, P_{n}^{(i)}\right)$ with $i \geqslant 1$ we have that $P_{1}^{(i)}=F_{i} \ldots F_{1}\left(P_{i+1}\right), \ldots, P_{n-i}^{(i)}=F_{i} \cdots F_{1}\left(P_{n}\right), P_{n-i+1}^{(i)}$ $=F_{i} \cdots F_{1}\left(\operatorname{Tr} D P_{1}\right), \ldots, P_{n-i+j}^{(i)}=F_{i} \cdots F_{j} \operatorname{Tr} D F_{j-1} \cdots F_{1}\left(P_{j}\right), \ldots, P_{n}^{(i)}$ $=F_{i} \operatorname{Tr} D F_{i-1} \cdots F_{1}\left(P_{i}\right)$.

Proof. Follows from the definition.

LEMMA 3.2. Given the pair $\left(\Lambda ; P_{1}, \ldots, P_{n}\right)$ the composition $F_{n} \cdots F_{1}$ of the functors $F_{i}: \bmod \Lambda_{i-1} \rightarrow \bmod \Lambda_{i}$ restricted to $\bmod _{P} \Lambda$ induces an equivalence of categories between $\bmod _{P} \Lambda$ and $\bmod _{I} \Lambda_{n}$.

Proof. We know that $F_{i} \mid \mathcal{C}_{P(i-1)}: \mathcal{C}_{P(i-1)} \rightarrow \mathscr{D}_{T_{i}}$ is an equivalence of categories, where $\mathcal{C}_{P(i-1)}$ and $\mathscr{D}_{T_{i}}$ are, respectively, the full subcategories of $\bmod \Lambda_{i-1}$ and $\bmod \Lambda_{i}$ whose objects do not contain $P_{1}^{(i-1)}$ and $T_{i}$ as a summand, respectively. Since $F_{i}\left(P_{1}^{(i-1)}\right)=0$, the module $F_{n} \cdots F_{1}\left(P_{i}\right)=$ $F_{n} \cdots F_{1}\left(F_{i-1} \cdots F_{1}\left(P_{i}\right)\right)$ which by Lemma 3.1 is equal to $F_{n} \cdots F_{i}\left(P_{1}^{(i-1)}\right)$, is zero. So $F_{n} \cdots F_{1}$ vanishes on projectives and the composition $F_{n} \cdots F_{1} \mid \bmod _{P} \Lambda: \bmod _{P} \Lambda \rightarrow \bmod _{I} \Lambda$ is fully faithful. We know by Theorem 2.3 that the modules $F_{n} \cdot \cdot F_{2}\left(T_{1}\right)$, $F_{n} \cdots F_{3}\left(T_{2}\right), \ldots, T_{n}$ are injective. Since there are $n$ indecomposable injective $\Lambda_{n}$-modules, and these are exactly the indecomposable $\Lambda_{n}$-modules that are not of the form $F_{n} \cdots F_{1}(X)$, for some $X$ in $\bmod \Lambda$, we have that $F_{n} \cdots F_{1} \mid \bmod _{P} \Lambda: \bmod _{P} \Lambda \rightarrow \bmod _{I} \Lambda$ is an equivalence of categories.

Proposition 3.3. Let $\Lambda$ be an indecomposable hereditary artin ring, $\alpha$ : $\bmod \Lambda \rightarrow \bmod \Lambda$ an equivalence of categories. If the restriction of $\alpha$ to $\bmod _{I} \Lambda$ is isomorphic to the identity, then $\alpha$ is isomorphic to the identity.

Proof. It has been proven in [5, Chapter III, Propositions 1.1 and 1.3], that if $\Lambda$ is an indecomposable hereditary artin algebra either $\Lambda$ has no projective injectives or is isomorphic to the lower triangular matrix ring $T_{n}(K)$ over a division algebra $K$. It is not hard to see that the same is true for an hereditary ring.

This remark enables us to divide the proof into two cases. The proof in both cases is based on the fact that $\alpha$ is isomorphic to the identity if it carries projectives to projectives and restricted to the full subcategory of projective modules is isomorphic to the identity. Since $\bmod _{I} \Lambda$ is closed under submod- 
ules and projective covers, we are done by Lemma 2.2 when $\Lambda$ has no nonzero projective injectives.

Assume now that $\Lambda \cong T_{n}(K)$, for a division ring $K$. Then $\Lambda$ has only one indecomposable projective injective module $P_{n}$, and the other indecomposable projective modules are $P_{i}=r^{n-i} P_{n}, i=1, \ldots, n-1$. Then $P_{i}$ is in $\bmod _{I} \Lambda$, for $i<n$. As already remarked, it is enough to show that the restriction of $\alpha$ to the full subcategory of projective objects is isomorphic to the identity. Let $\mu: \mathrm{id}\left|\bmod _{I} \Lambda \rightarrow \alpha\right| \bmod _{I} \Lambda$ be an isomorphism. Consider the inclusion $i: P_{n-1} \rightarrow P_{n}$, and the diagram:

$$
\begin{array}{ccc}
\operatorname{id}\left(P_{n-1}\right) & \mu_{P_{n-1}} & \alpha\left(P_{n-1}\right) \\
\downarrow & \rightarrow & \\
\operatorname{id}(i) & & \downarrow \alpha(i) \\
\operatorname{id}\left(P_{n}\right) & & \alpha\left(P_{n}\right)
\end{array}
$$

Choose $\mu_{P_{n}}: \operatorname{id}\left(P_{n}\right) \rightarrow \alpha\left(P_{n}\right)$ such that $\mu_{P_{n}} \operatorname{id}(i)=\alpha(i) \mu_{P_{n-1}}$. This is possible, because $\alpha\left(P_{n}\right)$ is injective. Now any map $f: P_{j} \rightarrow P_{n}$ factors as $P_{j} \stackrel{f}{\rightarrow} P_{n-1} \stackrel{j}{\rightarrow} P_{n}$, so $\alpha(f) \mu_{P_{j}}=\mu_{P_{n}}$ id(f). Let now $f: P_{n} \rightarrow P_{n}$ be a map. One can prove that the diagram

$$
\begin{array}{lll}
\operatorname{id}\left(P_{n}\right) & \stackrel{\mu_{P_{n}}}{\rightarrow} & \alpha\left(P_{n}\right) \\
\operatorname{id}(f) \downarrow & & \downarrow \alpha(f) \\
\operatorname{id}\left(P_{n}\right) & \stackrel{\mu_{P_{n}}}{\rightarrow} & \alpha\left(P_{n}\right)
\end{array}
$$

commutes, using the following facts: $\mu_{P_{n}} \mathrm{id}(i)=\alpha(i) \mu_{P_{n-1}}, \mu: \mathrm{id} \mid \bmod _{I} \Lambda \rightarrow$ $\alpha \mid \bmod _{I} \Lambda$ is a morphism of functors, and $\operatorname{End}_{\Lambda}\left(P_{n}\right)$ is a division ring.

We have the following consequence of the above proposition.

COROllary 3.4. Let $K$ be a division ring. Then all the automorphisms of $T_{n}(K)$ are inner.

Proof. Let $\Phi: T_{n}(K) \rightarrow T_{n}(K)$ be a ring isomorphism. Then the equivalence of categories $\bar{\Phi}: \bmod T_{n}(K) \rightarrow \bmod T_{n}(K)$ induced by $\Phi$ is isomorphic to the identity if and only if $\Phi$ is inner (see [15, pp. 454-456]). So it is enough to prove that every equivalence $\sigma: \bmod T_{n}(K) \rightarrow \bmod T_{n}(K)$ is isomorphic to the identity. To prove this we proceed by induction on $n$. The result is true if $n=1$. If $n>1$, the restriction of $\Phi$ to $\bmod _{l} \Lambda$ induces an equivalence $\Phi \mid \bmod _{I} \Lambda: \bmod _{I} \Lambda \rightarrow \bmod _{I} \Lambda$. Since $\bmod _{I} \Lambda$ is equivalent to $\bmod T_{n-1}(K)$ we have a commutative diagram

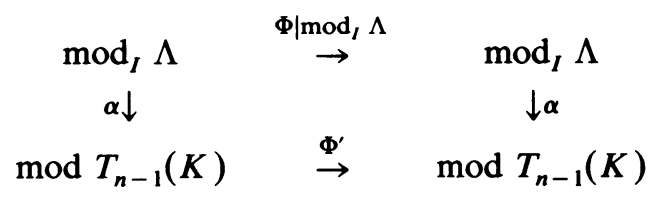


where $\alpha$ and $\Phi^{\prime}$ are equivalences. By the induction hypothesis $\Phi^{\prime}$ is isomorphic to the identity, so $\Phi \mid \bmod _{I} \Lambda$ is also isomorphic to the identity. Then it follows, by Proposition 3.3, that $\sigma$ is isomorphic to the identity. This ends the proof of the corollary.

LEMMA 3.5. Let $\Lambda_{1}, \Lambda_{2}$ be indecomposable hereditary artin rings which are not simple and let $\tau: \bmod _{I} \Lambda_{1} \rightarrow \bmod _{I} \Lambda_{2}$ be an equivalence of categories. Then there is an equivalence $\alpha: \bmod \Lambda_{1} \rightarrow \bmod \Lambda_{2}$ whose restriction to $\bmod _{1} \Lambda_{1}$ is $\tau$.

Proof. Since $\bmod _{I} \Lambda$ is closed under submodules we know by Lemma 2.2 that a $\Lambda_{1}$-module $P$ is projective noninjective if and only if $\alpha(P)$ is projective. Since $\tau$ has an inverse, it follows that the number of indecomposable projective noninjective $\Lambda_{1}$-modules is the same as the number of indecomposable projective noninjective $\Lambda_{2}$-modules, and the restriction of $\tau$ to the full subcategory $\mathscr{P}_{1}$ of projective noninjective $\Lambda_{1}$-modules induces an equivalence of categories between $\mathscr{P}_{1}$ and the full subcategory $\mathscr{P}_{2}$ of projective noninjective $\Lambda_{2}$-modules.

To prove the lemma it is enough, as before, to consider the case when $\Lambda_{1}$ has no nonzero projective injectives and the case when $\Lambda_{1}=T_{n}(K)$.

Case 1. There are no nonzero projective injective $\Lambda_{1}$-modules. If $\Lambda_{2}$ has a nonzero projective injective module, then $\Lambda_{2} \cong T_{m}(K)$, for a division ring $K$. Then $\mathscr{P}_{2}$ is equivalent to the full subcategory of $\bmod T_{m-1}(K)$ of projective modules, and $\alpha: \mathscr{P}_{1} \rightarrow \mathscr{P}_{2}$ induces an equivalence of categories between $\bmod \Lambda$ and $\bmod T_{m-1}(K)$. Therefore $\Lambda$ is isomorphic to $T_{m-1}(K)$, because both rings are basic, and this contradicts the assumption that there are no nonzero projective injective $\Lambda_{1}$-modules. This proves that there are no nonzero projective injective $\Lambda_{2}$-modules, so $\tau \mid \mathscr{P}_{1}: \mathscr{P}_{1} \rightarrow \mathscr{P}_{2}$ induces an equivalence $\alpha: \bmod \Lambda_{1} \rightarrow \bmod \Lambda_{2}$. If $M$ is in $\bmod _{I} \Lambda$ and $0 \rightarrow P_{1} \rightarrow P_{0} \rightarrow M \rightarrow 0$ is a projective resolution of $M$, we have that $\tau\left(P_{1}\right) \rightarrow \tau\left(P_{0}\right) \rightarrow \tau(M) \rightarrow 0$ is exact by Lemma 2.2. This shows that $\alpha$ extends $\tau$ and finishes the proof of the lemma in this case.

Case 2. Assume now that $\Lambda_{1}=T_{n}(K)$, for a division ring $K$. If $\Lambda_{2}$ has no nonzero projective injective modules then we know by the first case that $\Lambda_{1}$ and $\Lambda_{2}$ are isomorphic, and this is a contradiction. So $\Lambda_{2} \simeq T_{m}\left(K^{\prime}\right)$, where $K^{\prime}$ is a division ring. We will see now that $K \cong K^{\prime}$. We recall that $K$ is the endomorphism ring of an arbitrary indecomposable $\Lambda_{1}$-module, $K^{\prime}$ is the endomorphism ring of an arbitrary indecomposable $\Lambda_{2}$-module. So let $A$ be a nonzero indecomposable noninjective $\Lambda_{1}$-module. Since $\tau: \bmod _{I} \Lambda_{1} \rightarrow$ $\bmod _{I} \Lambda_{2}$ is an equivalence of categories, then $\operatorname{End}_{\Lambda}(A) \cong \operatorname{End}_{\Lambda}(\tau A)$, so $K \cong K^{\prime}$. Since $\alpha \mid \mathscr{P}_{1}: \mathscr{P}_{1} \rightarrow \mathscr{P}_{2}$ is an equivalence and every ring has only one indecomposable projective injective module, then $n-1=m-1$, so $n=m$. The map $\varphi: T_{n}(K) \rightarrow T_{n-1}(K)$ defined by 


$$
\varphi\left(\begin{array}{ccccc}
a_{11} & 0 & & \cdots & 0 \\
a_{21} & a_{22} & 0 & \cdots & 0 \\
\vdots & & & & \vdots \\
a_{n 1} & & & \cdots & a_{n n}
\end{array}\right)=\left(\begin{array}{cccc}
a_{22} & 0 & \cdots & 0 \\
\vdots & & & \vdots \\
a_{n 2} & & \cdots & a_{n n}
\end{array}\right)
$$

induces a fully faithful functor $\bar{\varphi}: \bmod T_{n-1}(K) \rightarrow \bmod T_{n}(K)$ whose image is $\bmod _{I}\left(T_{n}(K)\right)$. So it is enough to prove that given any equivalence $\beta$ : $\bmod$ $T_{n-1}(K) \rightarrow \bmod T_{n-1}(K)$ there is an equivalence $\bar{\beta}: \bmod T_{n}(K) \rightarrow$ $\bmod T_{n}(K)$ such that $\bar{\beta} \bar{\varphi}=\bar{\varphi} \beta$. So let $\beta: \bmod T_{n-1}(K) \rightarrow \bmod T_{n-1}(K)$ be an equivalence, and let $\rho: T_{n-1}(K) \rightarrow T_{n-1}(K)$ be the ring isomorphism defining $\beta$, that is, such that $\bar{\rho}=\beta$. We know by Corollary 3.4 that $\rho$ is inner. So $\rho(x)=A x A^{-1}$, for some $A \in T_{n-1}(K)$, and for all $x \in T_{n-1}(K)$. Let $\rho^{\prime}$ : $T_{n}(K) \rightarrow T_{n}(K)$ be the automorphism defined by conjugation by $\left(\begin{array}{ll}1 & 0 \\ 0 & A\end{array}\right)$. Then the equivalence $\bar{\beta}=\bar{\rho}^{\prime}: \bmod T_{n}(K) \rightarrow \bmod T_{n}(K)$ verifies the required condition.

We can now prove the following result.

Proposition 3.6. Let $\Lambda, \Lambda_{1}, \Lambda_{2}$, be hereditary artin rings with no semisimple ring summands, let $G: \bmod _{P} \Lambda \rightarrow \bmod _{I} \Lambda_{1}, H: \bmod _{P} \Lambda \rightarrow \bmod _{I} \Lambda_{2}$ be equivalences of categories. Then:

(a) There is a ring isomorphism $\phi: \Lambda_{2} \rightarrow \Lambda_{1}$ such that $\bar{\phi} G=H$.

(b) $\phi^{\prime}: \Lambda_{2} \rightarrow \Lambda_{1}$ is a ring isomorphism such that $\bar{\phi}^{\prime} G$ is isomorphic to $H$ if and only if $\phi^{\prime}=\alpha \phi$, where $\alpha$ is an inner automorphism of $\Lambda_{2}$.

Proof. (a) Follows from Lemma 3.5.

(b) Follows from Proposition 3.3 and from the fact that if $\alpha: \Lambda \rightarrow \Lambda$ is a ring isomorphism, then the equivalence $\bar{\alpha}: \bmod \Lambda \rightarrow \bmod \Lambda$ defined by $\alpha$ is isomorphic to the identity if and only if $\alpha$ is inner.

As an easy consequence of Proposition 3.6 we obtain the following important result.

THEOREM 3.7. We use the notations of the beginning of this section. Given a pair $\left(\Lambda ; P_{1}, \ldots, P_{n}\right)$ where $n>1$ there is a ring isomorphism $\phi: \Lambda_{n} \rightarrow \Lambda$ such that the functors $\bar{\phi} F_{n} \ldots F_{1}$ and $D$ Tr are isomorphic. Moreover, $\phi^{\prime}: \Lambda_{n} \rightarrow \Lambda$ is another ring isomorphism such that $\bar{\phi}^{\prime} F_{n} \ldots F_{1}$ and $D \operatorname{Tr}$ are isomorphic if and only if $\phi^{\prime}=\alpha \phi$, where $\alpha$ is an inner automorphism of $\Lambda$.

Proof. By Lemma 3.2 we know that $F_{n} \cdots F_{1}: \bmod _{P} \Lambda \rightarrow \bmod _{I} \Lambda$ is an equivalence of categories. We also know that $D \operatorname{Tr}: \bmod _{P} \Lambda \rightarrow \bmod _{I} \Lambda$ is an equivalence of categories. Then Theorem 3.7 is obtained by applying Proposition 3.6 to these two equivalences.

In view of this result, in order to describe the isomorphisms $\phi: \Lambda \rightarrow \Lambda_{n}$ which have the property that $\bar{\phi} F_{n} \cdots F_{1} \cong D \operatorname{Tr}$ it is enough to give one 
isomorphism from $\Lambda$ to $\Lambda_{n}$ with this property.

To do this it is enough, as we remarked above, to consider the case when $\Lambda$ has no indecomposable projective injective modules and the case when $\Lambda \cong T_{n}(K)$.

Case $1 . \Lambda$ has no indecomposable projective injective modules.

If $U$ is an indecomposable noninjective $\Lambda_{i-1}$-module not isomorphic to the simple projective $\Lambda_{i-1}$-module $F_{i-1} \cdots F_{1}\left(P_{i}\right)$, then we know by Proposition 2.1 that $F_{i}(U)$ is not injective and $F_{i}(\operatorname{Tr} D U) \cong \operatorname{Tr} D F_{i}(U)$. Although these isomorphisms are not natural, once they have been chosen the rest of the construction of the isomorphism $\psi: \Lambda \rightarrow \Lambda_{n}$ is natural. Iterating this argument we have, since $P_{1}, \ldots, P_{n}$ are not injective, that $\operatorname{Tr} D F_{j} \cdots F_{1}\left(P_{j+1}\right) \simeq$ $F_{j} \cdots F_{1}\left(\operatorname{Tr} D P_{j+1}\right), j=1, \ldots, n-1$. From this and Lemma 3.1 we have that the nonisomorphic projective indecomposable $\Lambda_{n}$-modules are $F_{n} \cdots F_{1}\left(\operatorname{Tr} D P_{i}\right), i=1, \ldots, n$. Since $\Lambda_{n}$ is basic, $\Lambda_{n}$ is isomorphic, as a $\Lambda_{n}$-module, to $F_{n} \cdots F_{1}(\operatorname{Tr} D \Lambda)$. Let $\psi$ be the composition of the natural ring isomorphisms

$$
\begin{aligned}
\Lambda_{n} & \cong \operatorname{End}_{\Lambda_{n}}\left(\Lambda_{n}\right)^{\mathrm{op}} \cong \operatorname{End}_{\Lambda_{n}}\left(F_{n} \cdots F_{1}(\operatorname{Tr} D \Lambda)\right) \\
& \cong \operatorname{End}_{\Lambda}(\operatorname{Tr} D \Lambda) \stackrel{D \operatorname{Tr}}{\cong} \operatorname{End}_{\Lambda}(\Lambda),
\end{aligned}
$$

where the last map is an isomorphism because $\Lambda$ is in $\bmod _{I} \Lambda$ and $D \operatorname{Tr}$ : $\bmod _{P} \Lambda \rightarrow \bmod _{I} \Lambda$ is an equivalence of categories.

Both $\bar{\psi} F_{n} \cdots F_{1}$ and $D \operatorname{Tr}$ are functors from $\bmod \Lambda$ to itself that are zero on projectives, so to prove that they are isomorphic we consider $M$ in $\bmod _{P} \Lambda$. There are functorial group isomorphisms

$$
\begin{aligned}
F_{n} \cdots F_{1}(M) & \cong \operatorname{Hom}_{\Lambda_{n}}\left(\Lambda_{n}, F_{n} \cdots F_{1}(M)\right) \\
& \cong \operatorname{Hom}_{\Lambda_{n}}\left(F_{n} \cdots F_{1}(\operatorname{Tr} D \Lambda), F_{n} \cdots F_{1}(M)\right) .
\end{aligned}
$$

Since $\operatorname{Tr} D \Lambda$, and $M$ are in $\bmod _{P} \Lambda$ and $F_{n} \cdots F_{1} \mid \bmod _{P} \Lambda$ is fully faithful we have that

$$
\begin{aligned}
\operatorname{Hom}_{\Lambda_{n}}\left(F_{n} \cdots F_{1}(\operatorname{Tr} D \Lambda), F_{n} \cdots F_{1}(M)\right) \\
\cong \operatorname{Hom}_{\Lambda}(\operatorname{Tr} D \Lambda, M) \stackrel{D \operatorname{Tr}}{\cong} \operatorname{Hom}_{\Lambda}(\Lambda, D \operatorname{Tr} M) \simeq D \operatorname{Tr} M .
\end{aligned}
$$

With a straightforward verification using the definition of $\bar{\psi}$ one can now check that the composition $\delta(M): \bar{\psi} F_{n} \cdots F_{1}(M) \rightarrow D \operatorname{Tr} M$ of the isomorphisms described above is a $\Lambda$-module homomorphism. Since $\delta$ is functorial in $M$, we have that $\psi$ verifies the desired conditions.

Case 2. $\Lambda \cong T_{n}(K)$. Then $P_{n}$ is the only indecomposable projective injective module, and is the injective envelope of the simple projective module $P_{1}$. So $F_{1}\left(P_{n}\right) \cong \operatorname{Tr} D T$, where $T$ is the simple injective $\operatorname{End}_{\Lambda}\left(\amalg_{2}^{n} P_{i} \amalg \operatorname{Tr} D P_{1}\right)^{\text {op }}$ module that is not in the image of $F_{1}$ (see Proposition 2.1). Therefore $F_{1}\left(P_{n}\right)$ is 
not injective. Since the modules $P_{1}, \ldots, P_{n-1}$ are not injective either, we know that $\operatorname{Tr} D F_{j} \cdots F_{1}\left(P_{j+1}\right) \cong F_{j} \cdots F_{1}\left(\operatorname{Tr} D P_{j+1}\right), j=1, \ldots, n-2$, and $\operatorname{Tr} D F_{j} \cdots F_{1}\left(P_{n}\right) \cong F_{j} \cdots F_{2}\left(\operatorname{Tr} D F_{1}\left(P_{n}\right)\right)$, for $j<n$. To prove this we use, as in Case 1, Proposition 2.1. $\Lambda_{n}$ is isomorphic, as a $\Lambda_{n}$-module, to the sum of one copy of each of the indecomposable projective $\Lambda_{n}$-modules. That is,

$$
\Lambda_{n} \cong \coprod_{i=1}^{n-1} F_{n} \cdots F_{1}\left(\operatorname{Tr} D P_{i}\right) \amalg F_{n} \cdots F_{2}\left(\operatorname{Tr} D F_{1}\left(P_{n}\right)\right) .
$$

We write $Q=\amalg_{i=1}^{n-1} P_{i}$. Then $\Lambda \cong P_{n} \amalg Q$,

$$
\Lambda_{n} \approx F_{n} \cdots F_{2} \operatorname{Tr} D F_{1}\left(P_{n}\right) \amalg F_{n} \cdots F_{1}(\operatorname{Tr} D Q),
$$

and there is a natural ring isomorphism

$$
\varphi: \operatorname{End}_{\Lambda}(Q) \rightarrow \operatorname{End}_{\Lambda_{n}}\left(F_{n} \cdots F_{1} \operatorname{Tr} D Q\right),
$$

defined by $\varphi(f)=F_{n} \cdots F_{1} \operatorname{Tr} D(f)$. By hypothesis, the ring $\Lambda$ is isomorphic to $T_{n}(K)$, so $\Lambda_{n}$ is also isomorphic to $T_{n}(K)$, by Theorem 3.6. There are then ring isomorphisms $\pi: \Lambda \rightarrow T_{n}(K), \pi^{\prime}: \Lambda_{n} \rightarrow T_{n}(K)$ such that

$$
\pi\left(\operatorname{End}_{\Lambda}(Q)^{\mathrm{op}}\right)=\pi^{\prime}\left(\operatorname{End}_{\Lambda_{n}}\left(Q^{\prime}\right)^{\mathrm{op}}\right)=\left\{\left(\begin{array}{cc}
A & 0 \\
0 & 0
\end{array}\right), A \in T_{n-1}(K)\right\} .
$$

Then $\pi^{\prime} \varphi \pi^{-1}$ can be considered as an automorphism of $T_{n-1}(K)$ and is, therefore, inner, by Corollary 3.4 , and is given by conjugation by some element $A_{1} \in T_{n-1}(K)$. Let $\alpha$ be the inner automorphism of $T_{n}(K)$ defined by conjugation by the matrix $\left(\begin{array}{c}A_{1} \\ 0\end{array}\right)$. We will now prove that $\bar{\alpha} F_{n} \cdots F_{1} \cong D \operatorname{Tr}$. As we recalled above, $\operatorname{Tr} D F_{1}\left(P_{n}\right) \cong T$ is simple injective. Therefore, $\operatorname{Hom}_{\Lambda_{1}}\left(\operatorname{Tr} D F_{1}\left(P_{n}\right), F_{1}(M)\right)=0$, because $F_{1}(M) \cong T$. On the other hand, $\operatorname{Hom}_{\Lambda}\left(P_{n}, D \operatorname{Tr} M\right)=0$, because $P_{n}$ is injective and therefore all its factors are injective. So $F_{n} \cdots F_{1}(M)$ and $D \operatorname{Tr} M$ are, respectively, modules over $\operatorname{End}_{\Lambda_{n}}\left(F_{n} \cdots F_{1}(Q)\right)^{\text {op }}$ and $\operatorname{End}_{\Lambda}(Q)^{\text {op }}$, and we can proceed as in Case 1. $\delta(M)$ is the composition of the group isomorphisms

$$
\begin{aligned}
\operatorname{Hom}_{\Lambda_{1}}\left(F_{1}(\operatorname{Tr} D Q), F_{1}(M)\right) & \simeq \operatorname{Hom}_{\Lambda}(\operatorname{Tr} D Q, M) \\
& \cong \operatorname{Hom}_{\Lambda}(Q, \operatorname{Tr} D M) \simeq \operatorname{Hom}_{\Lambda}(\Lambda, D \operatorname{Tr} M) \cong D \operatorname{Tr} M .
\end{aligned}
$$

One can again verify that $\delta(M): \bar{\psi} F_{n} \cdots F_{1}(M) \rightarrow D \operatorname{Tr} M$ is a $\Lambda$-homomorphism. Since $\delta$ is functorial in $M$, this ends our discussion.

4. Functors between modules over triangular matrix rings. In this section we study certain functors $F^{\prime}, G^{\prime}$ between modules over triangular matrix rings, which induce equivalences between subcategories of module categories. Specializing our assumptions, our functors $F^{\prime}$ and $G^{\prime}$ will be partial Coxeter functors between left artin rings. We show that if $\Lambda$ is a basic artin ring with a simple projective noninjective $\Lambda$-module $S_{i}, \Lambda=P \amalg S_{1}, X=P \amalg T r D^{\prime} S_{1}$, where $D^{\prime} S_{1}=S_{1}^{*} / \mathbf{r} S_{1}^{*}$ and $\left(\operatorname{Tr} D^{\prime} S_{1}\right)^{*}=0$, then our functor $F^{\prime}$ will be 


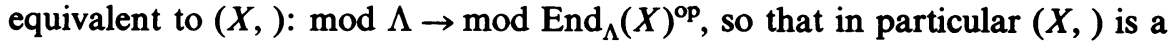
partial Coxeter functor.

We study functors between modules over triangular matrix rings of the form $\left(\begin{array}{ll}R & 0 \\ M\end{array}\right)$, where $R$ and $S$ are rings and ${ }_{S} M_{R}$ is an $S$-R-bimodule. We consider the (left) $\left(\begin{array}{cc}R & 0 \\ M\end{array}\right)$-modules as triples $(A, B, f)$, where $A$ is an $R$-module, $B$ is an $S$-module and $f: M \otimes A \rightarrow B$ is an $S$-homomorphism [16]. A morphism between two objects $(A, B, f)$ and $\left(A^{\prime}, B^{\prime}, f^{\prime}\right)$ is a pair $(\alpha, \beta)$, where $\alpha: A \rightarrow A^{\prime}$ is an $R$-homomorphism and $\beta: B \rightarrow B^{\prime}$ is an $S$-homomorphism such that the diagram

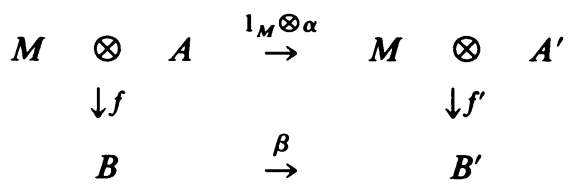

commutes. When it is more convenient we consider the $\left(\begin{array}{cc}R & 0 \\ M & S\end{array}\right)$-modules as triples $(g, U, V)$, where $U$ is an $R$-module, $V$ an $S$-module and $g: U \rightarrow$ $\operatorname{Hom}_{S}(M, V)$ is an $R$-homomorphism (see [16]). A morphism between two objects $(g, U, V)$ and $\left(g^{\prime}, U^{\prime}, V^{\prime}\right)$ is then a pair $(\alpha, \beta)$, where $\alpha: U \rightarrow U^{\prime}$ is an $R$-homomorphism, $\beta: V \rightarrow V^{\prime}$ is an $S$-homomorphism such that the diagram

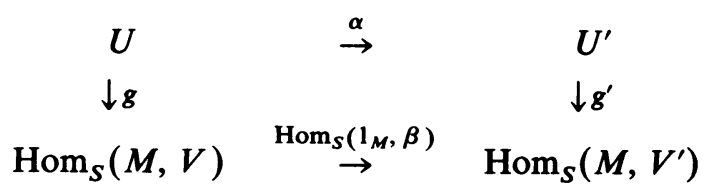

commutes. If ${ }_{s} M$ is finitely generated projective, then there is an isomorphism $\varphi$ between the functors $\operatorname{Hom}_{S}(M, S) \otimes$ and $\operatorname{Hom}_{S}(M$,$) from \operatorname{Mod} S$ to $\operatorname{Mod} R$, where $\varphi_{X}: \operatorname{Hom}_{S}(M, S) \otimes X \rightarrow \operatorname{Hom}_{S}(M, X)$ is given by $\left[\varphi_{X}(h \otimes\right.$ $x)](m)=h(m) x$, for $m \in M, h \in \operatorname{Hom}_{S}(M, S)$ and $x \in X$. Hence the modules can be considered as triples $(g, U, V)$, where $g: U \rightarrow M^{*} S \otimes_{S} V$ is an $R$-map. Here $M^{* S}$ denotes the $R$-S-bimodule $\operatorname{Hom}_{S}(M, S)$, and for a ring $\Delta$, Mod $\Delta$ denotes the category of all (left) $\Delta$-modules. A morphism between two objects $(g, U, V)$ and $\left(g^{\prime}, U^{\prime}, V^{\prime}\right)$ is then a pair of morphisms $(\gamma, \delta)$, where $\gamma: U \rightarrow U^{\prime}$ is an $R$-homomorphism and $\delta: V \rightarrow V^{\prime}$ is an $S$-homomorphism such that the diagram

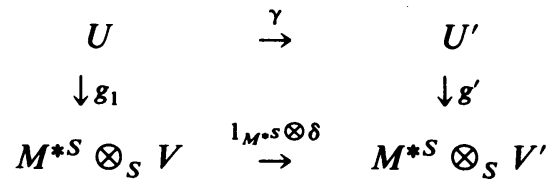


commutes. It will be clear from the context which description of the modules we use in each case.

Let now $R$ and $S$ be rings and ${ }_{S} M_{R}$ a bimodule such that $M_{R}$ is finitely generated and projective. Then ${ }_{R} N_{S}=M^{* R}$ is a finitely generated projective (left) $R$-module and $N^{* R}$ is isomorphic to ${ }_{S} M_{R}$.

Let $\Lambda=\left(\begin{array}{cc}R & 0 \\ M & S\end{array}\right)$ and $\Gamma^{\prime}=\left(\begin{array}{cc}S & 0 \\ N & R\end{array}\right)$. We shall now define a functor $F^{\prime}: \operatorname{Mod} \Lambda$ $\rightarrow \operatorname{Mod} \Gamma^{\prime}$, which for artin rings will be closely related to the functor $F$ we studied in $\S 1$. We shall also define a functor $G^{\prime}: \operatorname{Mod} \Gamma^{\prime} \rightarrow \operatorname{Mod} \Lambda$ which will be related to the functor $G$ defined in $\$ 1$.

For an $\left(\begin{array}{ll}R & 0 \\ M & S\end{array}\right)$-module $(A, B, f)$, define $F^{\prime}(A, B, f)$ to be $(j, \operatorname{Ker}(f), A)$, where $j: \operatorname{Ker}(f) \rightarrow M \otimes A$ is the natural morphism in the exact sequence $0 \rightarrow \operatorname{Ker}(f) \stackrel{j}{\rightarrow} M \otimes A \stackrel{f}{\rightarrow} B$. For a morphism $(\alpha, \beta):(A, B, f) \rightarrow\left(A^{\prime}, B^{\prime}, f^{\prime}\right)$, we define $F^{\prime}(\alpha, \beta)=(\gamma, \alpha)$, where $\gamma: \operatorname{Ker}(f) \rightarrow \operatorname{Ker}\left(f^{\prime}\right)$ is induced by $1_{M} \otimes$ $\alpha: M \otimes A \rightarrow M \otimes A^{\prime}$.

We define the functor $G^{\prime}: \operatorname{Mod}\left(\begin{array}{cc}S & 0 \\ N & R\end{array}\right) \rightarrow \operatorname{Mod}\left(\begin{array}{ll}R & 0 \\ M\end{array}\right)$ as follows, $G^{\prime}(g, U, V)$ $=(V, \operatorname{Coker}(g), p)$, where $p: M \otimes V \rightarrow \operatorname{Coker}(g)$ is the natural morphism such that $U \stackrel{g}{\rightarrow} M \otimes V \stackrel{p}{\rightarrow} \operatorname{Coker}(g) \rightarrow 0$ is exact. For a morphism $(\gamma, \delta)$ : $(g, U, V) \rightarrow\left(g^{\prime}, U^{\prime}, V^{\prime}\right)$, we define $G(\gamma, \delta)=(\delta, \beta):(V, \operatorname{Coker}(g), p) \rightarrow$ $\left(V^{\prime}, \operatorname{Coker}\left(g^{\prime}\right), p^{\prime}\right)$, where $\beta: \operatorname{Coker}(g) \rightarrow \operatorname{Coker}\left(g^{\prime}\right)$ is induced by $1_{M} \otimes \delta$ : $M \otimes V \rightarrow M \otimes V^{\prime}$.

We denote by $C$ the full subcategory of $\operatorname{Mod}\left({ }_{M}^{R} \stackrel{0}{S}\right)$ whose objects are the $(A, B, f)$, where $f: M \otimes A \rightarrow B$ is an epimorphism, and by $\mathscr{D}$ the full subcategory of $\operatorname{Mod}\left(\begin{array}{ll}S & 0 \\ N\end{array}\right)$ whose objects are the $(g, U, V)$ where $g: U \rightarrow M$ $\otimes V$ is a monomorphism.

We have the following relationship between the functors $F^{\prime}$ and $G^{\prime}$.

Proposition 4.1. Let the notation and assumptions be as above.

(a) $F^{\prime}$ is a right adjoint functor to $G^{\prime}$, hence $F^{\prime}$ is left exact and $G^{\prime}$ is right exact.

(b) The natural transformation $G^{\prime} F^{\prime} \rightarrow I$ given by the adjointness of $F^{\prime}$ and $G^{\prime}$ is an isomorphism on an object $(A, B, f)$ if and only if $f: M \otimes A \rightarrow B$ is an epimorphism, and the natural transformation $I \rightarrow F^{\prime} G^{\prime}$ is an isomorphism on an object $(g, U, V)$ if and only if $g: U \rightarrow M \otimes V$ is a monomorphism.

(c) $F^{\prime}: \mathcal{C} \rightarrow \mathscr{D}$ and $G^{\prime}: \mathscr{D} \rightarrow \mathcal{C}$ are inverse equivalences of categories.

(d) If $0 \rightarrow X \rightarrow Y \rightarrow Z \rightarrow 0$ is an exact sequence in $\operatorname{Mod}\left({ }_{M}^{R}{ }_{S}^{0}\right)$, where $X$ lies in $\mathcal{C}$, then $0 \rightarrow F^{\prime}(X) \rightarrow F^{\prime}(Y) \rightarrow F^{\prime}(Z) \rightarrow 0$ is exact, and if $0 \rightarrow X \rightarrow Y \rightarrow Z$ $\rightarrow 0$ is an exact sequence in $\operatorname{Mod}\left({ }_{N}^{S} \begin{array}{l}S_{R} \\ )\end{array}\right)$ where $Z$ lies in $\mathscr{D}$, then $0 \rightarrow G^{\prime}(X) \rightarrow$ $G^{\prime}(Y) \rightarrow G^{\prime}(Z) \rightarrow 0$ is exact.

Proof. (a) Let $(A, B, f)$ be in $\operatorname{Mod} \Lambda$ and $(g, U, V)$ in Mod $\Gamma^{\prime}$. We want to define a natural isomorphism between $\operatorname{Hom}\left(G^{\prime}(g, U, V),(A, B, f)\right)$ and 
$\operatorname{Hom}\left((g, U, V), F^{\prime}(A, B, f)\right)$. A morphism from $G^{\prime}(g, U, V)=$ $\left(V, \operatorname{Coker}(g), f^{\prime}\right)$ to $(A, B, f)$ is a pair $(\alpha, \beta)$, where $\alpha: V \rightarrow A, \beta: \operatorname{Coker}(g) \rightarrow$ $B$ are such that the diagram

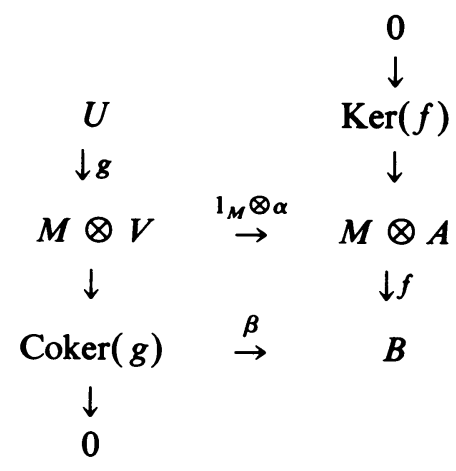

commutes. There is induced a morphism $\gamma: U \rightarrow \operatorname{Ker}(f)$ such that $(\gamma, \alpha)$ is a morphism from $(g, U, V)$ to $\left(g^{\prime}, \operatorname{Ker}(f), A\right)=F^{\prime}(A, B, f)$. It is not hard to see that in this way we get a natural isomorphism, so that $F^{\prime}$ is a right adjoint functor to $G^{\prime}$. Hence $F^{\prime}$ is left exact and $G^{\prime}$ is right exact.

(b) It is easy to see that $G^{\prime} F^{\prime}(A, B, f)=(A, \operatorname{Im}(f), f)$, and the natural morphism $(A, \operatorname{Im}(f), f) \rightarrow(A, B, f)$ is $\left(1_{A}, j\right)$, where $j: \operatorname{Im}(f) \rightarrow B$ is the inclusion morphism. From this it follows that the natural morphism $G^{\prime} F^{\prime}(A, B, f) \rightarrow(A, B, f)$ is an isomorphism if and only if $f: M \otimes A \rightarrow B$ is an epimorphism. Similarly $F^{\prime} G^{\prime}(g, U, V)=\left(g^{\prime}, \operatorname{Im}(g), V\right)$, where $g^{\prime}: \operatorname{Im}(g)$ $\rightarrow M \otimes V$ is the natural morphism induced by $g: U \rightarrow M \otimes V$, such that the diagram

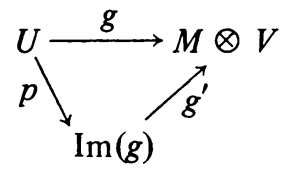

commutes. We then have the natural morphism $\left(p, 1_{V}\right)$ from $(g, U, V)$ to $\left(g^{\prime}, \operatorname{Im}(g), V\right)$, which is clearly an isomorphism if and only if $g: U \rightarrow M \otimes V$ is a monomorphism.

(c) Follows directly from (b), observing in addition that $F^{\prime}(A, B, f)$ is always in $\mathscr{D}$ and $G^{\prime}(g, U, V)$ is always in $\mathcal{C}$.

(d) Let $0 \rightarrow\left(A^{\prime}, B^{\prime}, f^{\prime}\right) \rightarrow(A, B, f) \rightarrow\left(A^{\prime \prime}, B^{\prime \prime}, f^{\prime \prime}\right) \rightarrow 0$ be an exact sequence in $\operatorname{Mod} \Lambda$, where $f^{\prime}: M \otimes A^{\prime} \rightarrow B^{\prime}$ is an epimorphism. That the above sequence is exact means that $0 \rightarrow A^{\prime} \rightarrow A \rightarrow A^{\prime \prime} \rightarrow 0$ and $0 \rightarrow B^{\prime} \rightarrow B$ $\rightarrow B^{\prime \prime} \rightarrow 0$ are exact sequences. Using that $M_{R}$ is projective and using the snake lemma, we get the following commutative diagram: 


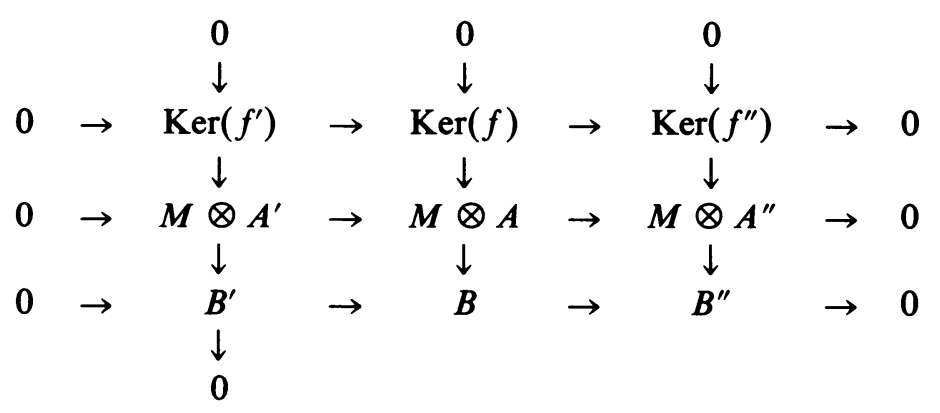

This shows that we get an exact sequence

$$
0 \rightarrow F^{\prime}\left(A^{\prime}, B^{\prime}, f^{\prime}\right) \rightarrow F^{\prime}(A, B, f) \rightarrow F^{\prime}\left(A^{\prime \prime}, B^{\prime \prime}, f^{\prime \prime}\right) \rightarrow 0 .
$$

The second part of (d) follows similarly.

Assume now that $R$ and $S$ are basic artin rings, not necessarily artin algebras, and that ${ }_{S} M_{R}$, in addition to being a finitely generated projective $R$-module, is a finitely generated $S$-module. Then $\Lambda=\left(\begin{array}{c}R \\ M\end{array} \underset{S}{0}\right)$ is an artin ring, and $\Gamma^{\prime}=\left(\begin{array}{cc}S & S^{* R} \\ R\end{array}\right)$ is a left artin ring. In this case it is easy to see that if $(A, B, f)$ is finitely generated, then $F^{\prime}(A, B, f)$ is finitely generated, so that $F^{\prime}$ induces a functor from $\bmod \Lambda$ to $\bmod \Gamma^{\prime}$. If we assume that $S$ is a division ring and $M$ is not zero, $F$ will be a (left) partial Coxeter functor in the sense of $\$ 2$, as follows from Proposition 4.1. For it is not hard to see that in this case the only indecomposable $\Lambda$-module not in $C$ is $S_{1}=(0, S, 0)$, which is simple projective noninjective, and the only indecomposable $\Gamma^{\prime}$-module not in $\mathscr{D}$ is $(S, 0,0)$, which is a simple injective nonprojective $\Gamma^{\prime}$-module.

If we start with a basic artin ring $\Lambda$ with a simple projective module $S_{1}$ and we write $\Lambda=S_{1} \amalg P$, then $\Lambda$ is isomorphic to the triangular matrix ring $\left(\begin{array}{cc}R & 0 \\ M & S\end{array}\right)$ where $R=\operatorname{End}_{\Lambda}(P)^{\text {op }}, S=\operatorname{End}_{\Lambda}\left(S_{1}\right)^{\text {op }}$ and $M=\operatorname{Hom}_{\Lambda}\left(S_{1}, P\right)$. Since we get a partial Coxeter functor when $M_{R}$ is finitely generated projective, we shall investigate when this condition is satisfied.

Proposition 4.2. Let $\Lambda$ be a basic artin ring with a simple projective noninjective module $S_{1}$ and let $P$ be given by $\Lambda=S_{1} \amalg P$. Then $M=\left(S_{1}, P\right)$ is a right projective $\operatorname{End}(P)^{\mathrm{op}}$-module if and only if $\left(\operatorname{Tr} D^{\prime} S_{1}\right)^{*}=0$, where as in

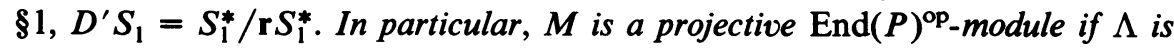
hereditary.

Proof. Let $0 \rightarrow S_{1} \rightarrow Q \rightarrow \operatorname{Tr} D^{\prime} S_{1} \rightarrow 0$ be an almost split sequence, as described at the end of $\$ 1$. Consider the exact sequence

$$
0 \rightarrow\left(\operatorname{Tr} D^{\prime} S_{1}, P\right) \stackrel{t}{\rightarrow}(Q, P) \rightarrow\left(S_{1}, P\right) \rightarrow \operatorname{Ext}_{\Lambda}^{1}\left(\operatorname{Tr} D^{\prime} S_{1}, P\right) \rightarrow 0 .
$$

Since $S_{1}$ is simple and not isomorphic to a summand of $P$, it follows, as for algebras in Lemma 1.1, that $\operatorname{Ext}_{\Lambda}^{1}\left(\operatorname{Tr} D^{\prime} S, P\right)$ is zero. Assume now that 
$\left(\operatorname{Tr} D^{\prime} S_{1}\right)^{*}=0$. Then we have an isomorphism $(Q, P) \rightarrow\left(S_{1}, P\right)$. Since every indecomposable summand of $Q$ is a summand of $P$, we conclude that $(Q, P)$, and hence $\left(S_{1}, P\right)$, is a projective $\operatorname{End}(P)$-module.

Assume conversely that $\left(S_{1}, P\right)$ is a projective $\operatorname{End}(P)$-module. Then there is a map $v:\left(S_{1}, P\right) \rightarrow(Q, P)$ such that $(Q, P)=\operatorname{Im}(t) \amalg \operatorname{Im}(v)$. Since $Q$ is in $\operatorname{add}(P)$ and $(, P)$ induces an equivalence of categories between $\operatorname{add}(P)$ and the finitely generated projective $\operatorname{End}(P)$-modules, there is a decomposition $Q=Q_{1} \amalg Q_{2}$ inducing the decomposition $(Q, P)=\left(Q_{1}, P\right) \amalg\left(Q_{2}, P\right)$, where $\left(Q_{1}, P\right)=\operatorname{Im}(t)$ and $\left(Q_{2}, P\right)=\operatorname{Im}(v)$. But then the induced morphism $\left(Q_{1}, P\right) \rightarrow\left(S_{1}, P\right)$ is zero. Since $Q_{1}$ is in $\operatorname{add}(P)$ this implies that the map $S_{1} \rightarrow Q_{1}$ is zero. This is a contradiction since the map $S_{1} \rightarrow Q_{1}$ is irreducible, because $Q_{1}$ is a summand of $Q$, and $0 \rightarrow S_{1} \rightarrow Q \rightarrow \operatorname{Tr} D^{\prime} S_{1} \rightarrow 0$ is an almost split sequence [7, Theorem 2.4]. Hence $\left(\operatorname{Tr} D^{\prime} S_{1}, P\right)$ is zero, and since obviously $\left(\operatorname{Tr} D^{\prime} S_{1}, S_{1}\right)$ is zero, we conclude that $\left(\operatorname{Tr} D^{\prime} S_{1}\right)^{*}=0$.

We can now combine our results so far in this section to get the following proposition, which in particular gives us examples of Coxeter functors between left artin rings which are not necessarily artin algebras.

Proposition 4.3. Let $\Lambda$ be a basic artin ring with a simple projective noninjective module $S_{1}$, let $\Lambda=S_{1} \amalg P$, and assume that $\left(\operatorname{Tr} D^{\prime} S_{1}\right)^{*}$ is zero. Then $\Lambda=\left(\begin{array}{cc}R & 0 \\ M\end{array}\right)$, where $R=\operatorname{End}(P)^{\text {op }}, S=\operatorname{End}\left(S_{1}\right)^{\text {op }}$ and $M_{R}=\left(S_{1}, P\right)_{R}$ is finitely generated projective. Let $\Gamma^{\prime}=\left(\begin{array}{c}S \\ M^{* R}\end{array}{ }_{R}^{0}\right)$. Then the functors $F^{\prime}: \bmod \Lambda \rightarrow$ $\bmod \Gamma^{\prime}$ and $G^{\prime}: \bmod \Gamma^{\prime} \rightarrow \bmod \Lambda$ are adjoint partial Coxeter functors which induce inverse equivalences of categories between $\mathcal{C}_{S_{1}}$ and $\mathscr{D}_{T}$, where $S_{1}=$ $(0, S, 0)$ and $T=(S, 0,0)$.

COROLlaRY 4.4. Let $\Lambda$ be a basic artin ring and $S_{1}$ a simple projective noninjective $\Lambda$-module such that $\left(\operatorname{Tr} D^{\prime} S_{1}\right)^{*}$ is zero, $\Lambda=P \amalg S_{1}, X=$ $P \amalg \operatorname{Tr} D^{\prime} S_{1}, \Gamma=\operatorname{End}(X)^{\text {op }}$. Then $(X):, \bmod \Lambda \rightarrow \bmod \Gamma$ is a left partial Coxeter functor.

Proof. By Proposition 4.3 there is some left partial Coxeter functor $F=F_{S}: \bmod \Lambda \rightarrow \bmod \Lambda^{\prime}$, which is by Theorem 2.3 equivalent to $(X$,$) ,$ which is then a left partial Coxeter functor.

5. Connections with diagrams. For a certain class of hereditary artin tensor rings, namely hereditary rings with duality conditions, partial Coxeter functors and Coxeter functors have been studied in [9] and [13], as a tool for studying the representation theory of these rings. Actually, these functors are defined between categories of representations of diagrams, but can be interpreted to give functors between module categories. They are left or right partial Coxeter functors in the sense of $\$ 2$, and are by our uniqueness results from $\$ 2$, closely related to the functors studied in $\$ \S 1$ and 4 . We shall see here 
that the connection with the triangular matrix case studied in $\$ 4$ is particularly natural. A Coxeter functor $C^{+}$in the diagram case is defined to be a composite of partial Coxeter functors defined for diagrams, and is, by our results in $\$ 2$, closely related to $D \mathrm{Tr}$ in the case of artin algebras. However, we can not conclude in general that $C^{+}$is isomorphic to $D \mathrm{Tr}$. In particular, $\mathrm{C}^{+}$is not uniquely defined up to isomorphism of functors, and we include a discussion of this problem. However, it has been shown in [10] that for an artin tensor algebra $\Lambda$ there is some Coxeter functor $C^{+}$which is isomorphic to $D$ Tr. Here we show that for an arbitrary tensor ring with duality conditions, a given Coxeter functor $C_{1}^{+}$is isomorphic to $D_{1} \operatorname{Tr}$ for some duality $D_{1}$.

For the convenience of the reader, we start out by recalling some background material from [9], [13].

A valued graph $(\Gamma, \mathbf{d})$ is a finite set $\Gamma$ of vertices together with pairs of nonnegative integers $\left(d_{i j}, d_{j i}\right)$ with $d_{i i}=0$ for all $i \in \Gamma$, and such that there exist natural numbers $f_{i}$ satisfying $d_{i j} f_{j}=d_{j i} f_{i}$ for all $i, j$ in $\Gamma$. We shall assume that $(\Gamma, \mathrm{d})$ is connected, in the sense that for every $k, t$ in $\Gamma$ there is a sequence $k, \ldots, i, j, \ldots, t$ of vertices of $\Gamma$ such that $d_{i j} \neq 0$ for each pair of subsequent vertices $i, j$. Pairs $\{i, j\}$ where $d_{i j} \neq 0$ are the edges and $i$ and $j$ are said to be neighbors.

An orientation $\Omega$ of a valued graph $(\Gamma, \mathbf{d})$ is given by prescribing for each edge $i, j$ of $(\Gamma, \mathrm{d})$ an order, indicated by an arrow $i \rightarrow j$. Given an orientation $\Omega$ and a vertex $k$ in $\Gamma$, we get a new orientation $s_{k} \Omega$ of $(\Gamma, \mathrm{d})$ by reversing the direction of arrows along all edges containing $k$. A vertex $k$ in $\Gamma$ is said to be a sink with respect to $\Omega$ if $k \leftarrow i$ for all neighbors $i \in \Gamma$ of $k$, and a source if $k \rightarrow i$ for all neighbors $i \in \Gamma$ of $k$. An orientation $\Omega$ of $(\Gamma, d)$ is said to be admissible if there is an ordering $k_{1}, \ldots, k_{n}$ of the vertices of $\Gamma$ such that each vertex $k_{r}$ is a sink with respect to the orientation $s_{k_{-1}} \cdots s_{k_{2}} s_{k_{1}} \Omega$ for all $r$, $1 \leqslant r \leqslant n$. Such an ordering of the $k_{i}$ is called an admissible ordering for $\Omega$.

A modulation $\mathbf{M}$ of a valued graph $(\Gamma, \mathrm{d})$ is a set of division rings $F_{i}, i \in \Gamma$, together with finite dimensional $F_{i}-F_{j}$-bimodules ${ }_{i} M_{j}$ and ${ }_{j} M_{i}$ for all edges $\{i, j\}$ of $(\Gamma, \mathbf{d})$ such that

(1) there are $F_{j}-F_{i}$-bimodule isomorphisms ${ }_{j} M_{i} \cong \operatorname{Hom}_{F_{i}}\left({ }_{i} M_{j}, F_{i}\right) \cong$ $\operatorname{Hom}_{F_{j}}\left({ }_{i} M_{j}, F_{j}\right)$,

(2) $\operatorname{dim}\left({ }_{i} M_{j}\right)_{F_{i}}=d_{i j}$.

A realization $(M, \Omega)$ of a valued graph $(\Gamma, d)$ is a modulation $M$ of $(\Gamma, d)$ together with an admissible orientation $\Omega$.

A representation $\mathbf{X}=\left({ }_{i} X, \varphi_{i j}\right)$ of a realization $(\mathbf{M}, \Omega)$ of $(\Gamma, \mathbf{d})$ is a set of finite dimensional left $F_{i}$-spaces ${ }_{i} X, i \in \Gamma$, together with $F_{j}$-linear maps ${ }_{j} \varphi_{i}$ : ${ }_{j} M_{i} \otimes_{F_{i}} X \rightarrow_{j} X$ for all oriented edges $i \rightarrow j$. A morphism from a representation $\mathbf{X}=\left({ }_{i} X,{ }_{j} \varphi_{i}\right)$ to a representation $\mathbf{X}^{\prime}=\left({ }_{i} X^{\prime},{ }_{j} \varphi_{i}^{\prime}\right)$ is a set $\alpha=\left(\alpha_{i}\right)$ of 
$F_{i}$-linear maps $\alpha_{i}:{ }_{i} X \rightarrow_{i} X^{\prime}$ for $i \in \Gamma$, such that ${ }_{j} \varphi_{i}^{\prime}\left(1_{j} M_{i} \otimes \alpha_{i}\right)=\alpha_{j}{ }_{j} \varphi_{i}$. The representations of $(M, \Omega)$ form an abelian category which we denote by $\mathcal{L}(\mathbf{M}, \Omega)$.

For a sink $k$ of the realization $(M, \Omega)$ there is a partial Coxeter functor $S_{k}^{+}$: $\mathcal{L}(\mathbf{M}, \Omega) \rightarrow \mathcal{L}\left(\mathbf{M}, s_{k} \Omega\right)$ defined as follows. Let $\mathbf{X}=\left({ }_{i} X,{ }_{j} \varphi_{i}\right)$ be an object in $\mathcal{L}(\mathbf{M}, \Omega)$. Then $S_{k}^{+}(\mathbf{X})$ is defined to be $\mathbf{Y}=\left({ }_{i} Y,{ }_{j} \psi_{i}\right)$, where ${ }_{i} Y={ }_{i} X$ for all $i \neq k$ and ${ }_{k} Y$ is the kernel in the sequence

$$
0 \rightarrow{ }_{k} Y \stackrel{\left(\pi_{k}\right)_{j}}{\rightarrow} \prod_{j \in \Gamma}{ }_{k} M_{j} \otimes_{j} X \stackrel{\left({ }_{k} \varphi_{j}\right)_{j}}{\rightarrow}{ }_{k} X .
$$

Further ${ }_{j} \psi_{i}={ }_{j} \varphi_{i}$ for $i \neq k$, and ${ }_{j} \psi_{k}={ }_{j} \bar{\pi}_{k}:{ }_{j} M_{k} \otimes{ }_{k} Y \rightarrow{ }_{j} Y$ is determined by ${ }_{j} \pi_{k}:{ }_{k} Y \rightarrow{ }_{k} M_{j} \otimes{ }_{j} Y$, using the natural isomorphism

$$
\operatorname{Hom}_{F_{j}}\left({ }_{j} M_{k} \otimes{ }_{k} Y,{ }_{j} Y\right) \rightarrow \operatorname{Hom}_{F_{k}}\left({ }_{k} Y, \operatorname{Hom}_{F_{j}}\left({ }_{j} M_{k},{ }_{j} Y\right)\right)
$$

and the inverse of the natural isomorphism

$$
\operatorname{Hom}_{F_{k}}\left({ }_{j} M_{k},{ }_{j} Y\right) \rightarrow \operatorname{Hom}_{F_{k}}\left({ }_{j} M_{k}, F_{k}\right) \otimes_{j} Y,
$$

together with the given isomorphism $\operatorname{Hom}_{F_{k}}\left({ }_{j} M_{k}, F_{k}\right) \rightarrow{ }_{k} M_{j} . S_{k}^{+}$is defined on morphisms in a natural way. Now a Coxeter functor $C^{+}: \mathcal{L}(\mathbf{M}, \Omega) \rightarrow$ $\mathcal{L}(\mathbf{M}, \Omega)$ is defined to be the composite functor $S_{k_{n}}^{+} \cdots S_{k_{1}}^{+}$, where $k_{1}, \ldots, k_{n}$ is an admissible ordering with respect to the orientation $\Omega$. The definition of $C^{+}$depends on the choice of bimodule isomorphisms. We shall see later in this section that different choices may give rise to nonisomorphic Coxeter functors.

For the convenience of the reader we also recall the connection between the categories $\mathcal{L}(\mathbf{M}, \Omega)$ and the categories of finitely generated modules over certain hereditary artin rings. (See [12], [18].) Assume that $(\Gamma, d)$ is a graph with no oriented cycles, and let $(M, \Omega)$ be a realization of $(\Gamma, d)$. Then there is a natural equivalence of categories between $\mathcal{L}(\mathbf{M}, \Omega)$ and $\bmod \Lambda$, where $\Lambda$ is the following hereditary basic artin ring. Let $i=1, \ldots, m$ be the vertices of $\Gamma$ and $F_{i}$ the corresponding division rings. Let $\Sigma=\prod_{i=1}^{m} F_{i}$, and let $\mathbf{r}=$ $\amalg_{j} M_{i}$, where ${ }_{j} M_{i}$ runs through all modules where there is an arrow $i \rightarrow j$. Since $(\Gamma, \mathbf{d})$ has no oriented cycles, the $n$-fold tensor product of $\mathbf{r}$ is zero for some smallest $n . \quad \Lambda$ is then the ring $\Sigma+\mathbf{r}+\mathbf{r} \otimes \mathbf{r}+\cdots+\mathbf{r} \otimes \cdots \otimes \mathbf{r}$, where the last term is an $(n-1)$-fold tensor product. Conversely, let $\Lambda$ be a basic hereditary artin ring of this form, where $\Sigma=\prod_{i=1}^{m} F_{i}$, and where we have a two-sided decomposition of $\mathrm{r}$ over $\Sigma$ as $\amalg_{j} M_{i}$, where we mean that for ${ }_{j} M_{i}$ only $F_{j}$ has a nonzero action on the left and only $F_{i}$ has a nonzero action on the right. Assuming that $\operatorname{Hom}_{F_{i}}\left({ }_{j} M_{i}, F_{i}\right)$ is isomorphic to $\operatorname{Hom}_{F_{j}}\left({ }_{j} M_{i}, F_{j}\right)$, we get a realization of a valued graph $(\Gamma, d)$, where $1,2, \ldots, m$ are the vertices of $\Gamma$, and there is an arrow $i \rightarrow j$ if and only if there is some module ${ }_{j} M_{i} \neq 0$ in the two-sided decomposition of $\mathbf{r}$. When an hereditary artin ring 
is of this type, we say that it is a tensor ring with duality conditions.

If $\mathbf{X}=\left({ }_{i} X,{ }_{i} \varphi_{j}\right)$ is in $\mathcal{L}(\mathbf{M}, \Gamma)$, then the corresponding $\Lambda$-module is $\amalg_{i} X$ as $\Sigma$-module, and the action of $\mathbf{r}=\amalg_{j} M_{i}$ on $\amalg_{i} X$ is given via the morphisms $i \varphi_{j}$.

If $\Lambda^{\prime}$ denotes the hereditary artin ring associated with $\left(\mathbf{M}, s_{k} \Omega\right)$, where $k$ is a sink for $\Omega$, then the partial Coxeter functor $S_{k}^{+}$defines a functor from mod $\Lambda$ to mod $\Lambda^{\prime}$. And for fixed bimodule isomorphisms the corresponding Coxeter functor $C^{+}$defines a functor from $\bmod \Lambda$ to $\bmod \Lambda$. By the properties of $S_{k}^{+}$ listed in [13, §2], we know that $S_{k}^{+}$is a left partial Coxeter functor in the sense defined in $\$ 2$. Hence we know by Theorem 2.3 that $S_{k}{ }^{+}$is equivalent to the other left partial Coxeter functors we have studied in this paper. We shall see here that we have a particularly natural connection between ${S_{k}}^{+}$and the left partial Coxeter functor defined for triangular matrix rings in $\$ 4$.

Let now $(M, \Omega)$ be a realization of a valued graph $(\Gamma, d)$ with no oriented cycles, let $k$ be a sink and let $1,2, \ldots, k-1$ be the other vertices. Let $R$ be the hereditary ring determined by the realization $\left(M^{\prime}, \Omega^{\prime}\right)$ of the valued graph $\left(\Gamma^{\prime}, \mathbf{d}^{\prime}\right)$ which we get by removing the vertex $k$ and the bimodules of the form ${ }_{k} M_{i}$ and ${ }_{i} M_{k}$. Let $K=\amalg_{i k} M_{i}, M=K \otimes_{\Sigma} R$ and $S=F_{k}$. Let $\mathrm{X}=\left({ }_{i} X, \varphi_{i j}\right)$ be a representation of $(\mathbf{M}, \Omega)$. Then $\mathbf{X}$ determines in a natural way a module $(A, B, f)$ over the triangular matrix ring $\left({ }_{S M_{R}}^{R}{ }_{S}^{0}\right)$, where the $R$-module $A$ is $\amalg_{i<k i} X$ as a $\Sigma$-module and the $R$-action is given by the $\varphi_{i j} . \quad B={ }_{k} X$, and the morphisms ${ }_{k} \varphi_{i}:{ }_{k} M_{i} \otimes{ }_{i} X \rightarrow{ }_{k} X$ determine a unique $S$-morphism $K$ $\otimes_{\Sigma} A \rightarrow B$, hence a morphism $f: M \otimes_{R} A \rightarrow B$, since we have a natural isomorphism $K \otimes_{\Sigma} R \otimes_{R} A \rightarrow K \otimes_{\Sigma} A$. It is not hard to see that we get a natural equivalence of categories $H_{1}: \mathcal{L}(\mathbf{M}, \Omega) \rightarrow \bmod \left({ }_{s M_{R}}^{R} \stackrel{0}{S}\right)$.

Let now $Y=\left({ }_{i} Y, \psi_{i j}\right)$ be a representation of $\left(\mathrm{M}, s_{k} \Omega\right)$. Let $C={ }_{k} Y, D$ the $R$-module which is $\amalg_{i<k}{ }_{i} Y$ as a $\Sigma$-module and the $R$-action is given by the $\psi_{i j}$. Then $(g, C, D)$ is a module over $\left({ }_{M^{* R}}^{S}{ }_{R}^{0}\right)$, where $g: C \rightarrow M \otimes D$ is given as follows. The morphisms ${ }_{j} \psi_{k}:{ }_{j} M_{k} \otimes{ }_{k} Y \rightarrow_{j} Y$ determine morphisms ${ }_{j} \pi_{k}$ : ${ }_{k} Y \rightarrow{ }_{k} M_{j} \otimes_{j} Y$, and hence determine morphisms $g: C \rightarrow \amalg_{j k} M_{j} \otimes_{j} Y=K$ $\otimes_{\Sigma} D=M \otimes_{R} D$. It is not hard to see that we get a natural equivalence of categories

$$
H_{2}: \mathcal{L}\left(\mathbf{M}, s_{k} \Omega\right) \rightarrow \bmod \left(\begin{array}{cc}
S & 0 \\
M^{* R} & R
\end{array}\right)
$$

Considering the definition of the functors $S_{k}^{+}: \mathcal{L}(\mathbf{M}, \Omega) \rightarrow \mathcal{L}\left(\mathbf{M}, s_{k} \Omega\right)$ and

$$
F^{\prime}: \bmod \left(\begin{array}{cc}
R & 0 \\
M & S
\end{array}\right) \rightarrow \bmod \left(\begin{array}{cc}
S & 0 \\
M^{* R} & R
\end{array}\right)
$$

it is not hard to see that we have a commutative diagram: 


$$
\begin{array}{ccc}
\mathcal{L}(\mathbf{M}, \Omega) & \stackrel{H_{1}}{\rightarrow} & \bmod \left(\begin{array}{cc}
R & 0 \\
M & S
\end{array}\right) \\
\downarrow S_{k}^{+} & & \downarrow F^{\prime} \\
\mathcal{L}\left(M, s_{k} \Omega\right) & \stackrel{H_{2}}{\rightarrow} & \bmod \left(\begin{array}{cc}
S & 0 \\
M^{* R} & R
\end{array}\right)
\end{array}
$$

We collect some of the above in the following proposition.

Proposition 5.1. Let $\Lambda$ be a tensor ring with duality conditions, and $S_{1} a$ simple projective noninjective $\Lambda$-module. Then the corresponding partial Coxeter functors $S_{1}^{+}$and $F^{\prime}$ from $\S 4$ are equivalent functors in a natural way.

Let $\Lambda$ be an hereditary tensor algebra. Then we know that a given Coxeter functor $C^{+}: \bmod \Lambda \rightarrow \bmod \Lambda$, which is by definition a composite of partial Coxeter functors, is equivalent to $D$ Tr by Theorem 3.6. For we know from $[13, \S 2]$ that $C^{+}$takes projectives to zero and induces an equivalence of categories $\bmod _{P} \Lambda \rightarrow \bmod _{I} \Lambda$. However, this does not necessarily mean that $D \mathrm{Tr}$ and $C^{+}$are isomorphic functors. In fact, the definition of $C^{+}$was made using fixed bimodule isomorphisms $\operatorname{Hom}_{F_{i}}\left({ }_{i} M_{j}, F_{i}\right) \rightarrow \operatorname{Hom}_{F_{j}}\left({ }_{i} M_{j}, F_{j}\right)$. As remarked in [13], the theory in [13] is independent of this choice. We shall see that different choices may give rise to nonisomorphic Coxeter functors. We shall, in fact, devote some time to a discussion of what this depends on.

Choosing bimodule isomorphisms $\operatorname{Hom}_{F_{i}}\left({ }_{i} \boldsymbol{M}_{j}, \boldsymbol{F}_{i}\right) \rightarrow \operatorname{Hom}_{\boldsymbol{F}_{j}}\left({ }_{i} \boldsymbol{M}_{j}, \boldsymbol{F}_{j}\right)$ is equivalent to choosing bimodule isomorphisms $\operatorname{Hom}_{F_{j}}\left(\left({ }_{i} M_{j}, F_{i}\right), F_{j}\right) \rightarrow{ }_{i} M_{j}$. Two different choices of these isomorphisms give rise to bimodule isomorphisms ${ }_{i} M_{j} \rightarrow{ }_{i} M_{j}$, which induce a ring isomorphism $t: \Lambda \rightarrow \Lambda$ by using the identity on the $F_{i}$. It is then not hard to see that if $C_{1}^{+}$and $C_{2}^{+}$are two Coxeter functors, then $C_{1}^{+}=t^{*} C_{2}^{+}$, where $t^{*}: \bmod \Lambda \rightarrow \bmod \Lambda$ is an equivalence of categories induced by a ring isomorphism which is given by bimodule isomorphisms ${ }_{i} M_{j} \rightarrow{ }_{i} M_{j}$. Since $C_{1}^{+}$and $C_{2}^{+}$both induce equivalences of categories between $\bmod _{P} \Lambda$ and $\bmod _{I} \Lambda$, it is easy to see that $C_{1}^{+}$and $C_{2}^{+}$are isomorphic if and only if the restriction of $t^{*}$ to $\bmod _{I} \Lambda$ is isomorphic to the identity.

We can then get the following result.

Proposition 5.2. Let $\Lambda$ be an artin hereditary tensor ring with duality conditions. Then the isomorphism classes of Coxeter functors are in one to one correspondence with the elements of the quotient group $G / H$, where $G$ is the group of automorphisms induced by bimodule isomorphisms ${ }_{i} \boldsymbol{M}_{j} \rightarrow{ }_{i} \boldsymbol{M}_{j}$, and $\boldsymbol{H}$ the subgroup consisting of those of this type which are inner.

Proof. Since an isomorphism $t: \Lambda \rightarrow \Lambda$ induces a functor $t^{*}: \bmod \Lambda \rightarrow$ $\bmod \Lambda$ which is isomorphic to the identity if and only if $t$ is an inner 
automorphism [15, pp. 454 456], this is a direct consequence of Proposition 3.3 and the above remarks.

As an immediate consequence we have the following.

COROLLARY 5.3. Let $\Lambda$ be an artin hereditary tensor ring with duality conditions. Then all Coxeter functors are isomorphic if and only if every ring isomorphism $\Lambda \rightarrow \Lambda$ induced by bimodule isomorphisms ${ }_{i} M_{j} \rightarrow{ }_{i} M_{j}$ is an inner automorphism.

To give a concrete example for when not all Coxeter functors are isomorphic consider $\Lambda=\left({ }_{K \amalg K}^{K}{ }_{K}^{0}\right)$, for a field $K$, where the $K$ - $K$-bimodule structure of $K \amalg K$ is given by $a(b, c)=(a b, a c),(b, c) d=(b d, c d)$. Then the isomorphism $t$ defined by

$$
t\left(\begin{array}{cc}
a & 0 \\
(b, c) & d
\end{array}\right)=\left(\begin{array}{cc}
a & 0 \\
(c, b) & d
\end{array}\right)
$$

is induced by a bimodule isomorphism $K \amalg K \rightarrow K \amalg K$, which is easily checked not to be inner. Hence by the above there are two Coxeter functors $\mathrm{C}_{1}^{+}$and $\mathrm{C}_{2}^{+}$which are not isomorphic.

Let

$$
P=\left(\begin{array}{cc}
K & 0 \\
K \amalg K & 0
\end{array}\right), \quad N_{1}=\left(\begin{array}{cc}
0 & 0 \\
K \amalg 0 & 0
\end{array}\right), \quad N_{2}=\left(\begin{array}{cc}
0 & 0 \\
0 \amalg K & 0
\end{array}\right) .
$$

Since $t^{*}\left(P / N_{1}\right)=P / N_{2}$ and $P / N_{1}, P / N_{2}$ are obviously not isomorphic, $C_{1}^{+}$ and $C_{2}^{+}$are also not isomorphic on objects.

We shall now give a direct interpretation of what a Coxeter functor $C^{+}$for an artin hereditary tensor ring $\Lambda$ looks like.

We have already seen that for artin algebras there is a close relationship between a given $C^{+}$and $D$ Tr.

We shall now see that if $C_{1}^{+}$is a Coxeter functor for an arbitrary hereditary tensor ring $\Lambda$ with duality conditions, then there is some duality $D_{1}: \bmod \Lambda^{\text {op }}$ $\rightarrow \bmod \Lambda$ such that $C_{1}^{+}$is isomorphic to $D_{1} \operatorname{Tr}$. In particular, this shows that the hereditary tensor rings $\Lambda$ with duality conditions have a duality. Our result will be a consequence of the following more general result.

THEOREM 5.4. Let $\Lambda$ and $\Lambda^{\prime}$ be indecomposable hereditary artin rings and $\alpha$ : $\bmod _{P} \Lambda \rightarrow \bmod _{I} \Lambda^{\prime}$ an equivalence of categories. Then $\alpha=D_{1} \operatorname{Tr} \mid \bmod _{P} \Lambda$ for some duality $D_{1}: \bmod \Lambda^{\mathrm{op}} \rightarrow \bmod \Lambda^{\prime}$.

Proof. Since Tr: $\bmod \Lambda^{\mathrm{op}} \rightarrow \bmod \Lambda$ induces a duality $\operatorname{Tr}: \bmod _{P} \Lambda^{\text {op }} \rightarrow$ $\bmod _{P} \Lambda$ we get a duality $\beta: \bmod _{P} \Lambda^{\mathrm{op}} \stackrel{\operatorname{Tr}}{\rightarrow} \bmod _{P} \Lambda \stackrel{\alpha}{\rightarrow} \bmod _{I} \Lambda^{\prime}$. Since $\bmod _{P} \Lambda^{\mathrm{op}}$ is closed under factors and $\bmod _{I} \Lambda^{\prime}$ is closed under subobjects, we can use Lemma 2.1 to conclude that a sequence $0 \rightarrow A \stackrel{f}{\rightarrow} B \stackrel{g}{\rightarrow} C \rightarrow 0$ with $A, B$ and $C$ in $\bmod _{P} \Lambda^{\text {op }}$ is exact if and only if $0 \rightarrow \beta C \stackrel{\beta g}{\rightarrow} \beta B \stackrel{\beta f}{\rightarrow} \beta A \rightarrow 0$ is exact. 
Let $A$ be indecomposable and not projective in $\bmod \Lambda^{\text {op }}$. If $A$ is injective, we can show as in Lemma 2.2 that $\beta(A)$ is projective since $\bmod _{I} \Lambda^{\prime}$ is closed under subobjects and projective covers, using that $\Lambda^{\prime}$ is hereditary. Assume next that $\beta(A)$ is projective. We will prove that then $A$ is injective. Let $i$ : $A \rightarrow B$ be a monomorphism where $B$ is in $\bmod _{P} \Lambda^{\text {op }}$, and consider the exact sequence $0 \rightarrow A \stackrel{i}{\rightarrow} B \rightarrow C \rightarrow 0$. Since $A, B$ and $C$ are in $\bmod _{P} \Lambda^{\text {op }}$, we know that $\beta(i): \beta(B) \rightarrow \beta(A)$ is an epimorphism, which splits since $\beta(A)$ is projective. Hence $i: A \rightarrow B$ is a split monomorphism. Using now the fact that all monomorphisms $i: A \rightarrow B$ in $\bmod _{P} \Lambda^{\text {op }}$ split we will prove that $A$ is injective. Let $j: K \rightarrow L$ be an arbitrary monomorphism in $\bmod \Lambda^{\mathrm{op}}$, and $t: K \rightarrow A$ a morphism. Consider the pushout diagram:

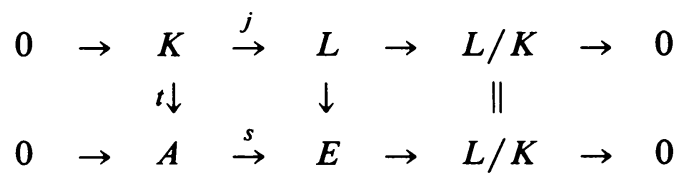

Since $A$ is not projective and $\Lambda^{\mathrm{op}}$ is hereditary, there is a decomposition $E=E_{1} \amalg E_{2}$ such that $\operatorname{Im}(s) \subset E_{1}$ and $E_{1}$ has no nonzero projective summands. Since $E$ and hence $E_{1}$ is finitely generated, we know that $A \rightarrow E_{1}$, and hence $s: A \rightarrow E$ is a split monomorphism. This shows that there is a map $u: L \rightarrow A$ such that $u j=t$, and it follows that $A$ is injective.

We point out that the above also shows that all indecomposable injectives in $\bmod \Lambda^{\mathrm{op}}$ are finitely generated. For let $A$ be indecomposable and not projective, and consider $0 \rightarrow \beta(K) \rightarrow \beta(B) \rightarrow \beta(A) \rightarrow 0$, where $\beta(B) \rightarrow \beta(A)$ is a projective cover. Then $0 \rightarrow A \rightarrow B$ is exact, and we have just seen that $B$ is then injective, so the injective envelope of $A$ is finitely generated. Let now $S$ be simple. If the injective envelope $I(S)$ of $S$ is not finitely generated then there is a finitely generated nonprojective module $E$ such that $S \subset E \subset I(S)$. By the above we know that the injective envelope of $E$, which is $I(S)$, is finitely generated, and this is a contradiction. So for every simple module $S$ the injective envelope of $S$ is finitely generated.

We can now conclude from the above that $\beta$ induces a duality between the finitely generated injective nonprojective $\Lambda^{\mathrm{op}}$-modules and the finitely generated projective noninjective $\Lambda$-modules. If $\Lambda$ and $\Lambda^{\prime}$ have no projective injectives, this can be extended to a duality $D_{1}: \bmod \Lambda^{\mathrm{op}} \rightarrow \bmod \Lambda^{\prime} . D_{1}$ extends $\beta$ since $\beta$ preserves exact sequences $0 \rightarrow A \rightarrow B \rightarrow C \rightarrow 0$ with $A, B$ and $C$ in $\bmod _{P} \Lambda^{\text {op }}$. This shows that $\alpha=D_{1} \operatorname{Tr}$ in this case.

If $\Lambda$ has a nonzero projective injective, we know that $\Lambda^{\text {op }}=T_{n}(K)$ for a division ring $K$. Then $\bmod _{P} \Lambda^{\mathrm{op}}$ is equivalent to $\bmod T_{n-1}(K)$, and we would get a duality between the finitely generated injective $T_{n-1}(K)$-modules and the finitely generated projective noninjective $\Lambda^{\prime}$-modules. If $\Lambda^{\prime}$ had no projective injectives we would get a duality between $\bmod T_{n-1}(K)$ and $\bmod \Lambda^{\prime}$, and 
get a contradiction. Hence we get that $\Lambda^{\prime}=T_{m}\left(K^{\prime}\right)$, where clearly $n=m$ and $K^{\prime}=K^{\text {op }}$. Let $Q$ be the projective injective indecomposable $\Lambda^{\prime}$-module and let $P$ be the projective injective indecomposable $\Lambda^{\mathrm{op}}$-module. We define $\beta(P)=Q$. Using an argument similar to that used in Proposition 3.3 one can extend the restriction of $\beta$ to the full subcategory of finitely generated injective nonprojective $\Lambda^{\mathrm{op}}$-modules to a duality $\beta$ from the full subcategory of finitely generated projective $\Lambda^{\mathrm{op}}$-modules to the full subcategory of finitely generated projective $\Lambda^{\prime}$-modules. Then, as in the first case, one can extend $\beta$ to a duality $D_{1} ; \bmod \Lambda^{\mathrm{op}} \rightarrow \bmod \Lambda^{\prime}$.

COROllaRY 5.5. Let $\Lambda$ be an hereditary artin tensor ring with duality conditions, and $C_{1}^{+}$a Coxeter functor. Then $C_{1}^{+}=D_{1} \operatorname{Tr}$ for some duality $D_{1}$.

Proof. This follows directly from Theorem 5.4, using that $C_{1}^{+}$and $D_{1} \operatorname{Tr}$ take projectives to zero.

Clearly we can not expect more specific information about the duality $D_{1}$ unless we have more information about the Coxeter functor $C_{1}^{+}$. It would be interesting to know in general how to describe the duality $D_{1}$ such that $C_{1}^{+}=D_{1} \operatorname{Tr}$ directly. Actually, the following gives a natural candidate for this duality $D_{1}$.

Let $(M, \Omega)$ be a realization corresponding to an hereditary tensor ring $\Lambda$ with duality conditions. As before, we denote by $F_{i}$ the associated division rings. Then the graph corresponding to $\Lambda^{\text {op }}$ has the same number of edges and arrows, all arrows have opposite direction, and the bimodules are the same, if we consider a representation as a collection of right $F_{i}$-spaces. Let $\mathbf{X}=\left({ }_{i} X, \varphi_{i j}\right)$ be a representation of $(\mathbf{M}, \Omega)$. We define $\mathbf{Y}=D_{1} \mathbf{X}$ to be the representation $\left(Y_{i},{ }_{i} \psi_{j}\right)$, where $Y_{i}=\operatorname{Hom}_{F_{i}}\left({ }_{i} X, F_{i}\right)$, and ${ }_{i} \psi_{j}: \operatorname{Hom}_{F_{i}}\left({ }_{i} X, F_{i}\right)$ $\otimes_{i} M_{j} \rightarrow \operatorname{Hom}_{F_{j}}\left({ }_{j} X, F_{j}\right)$ is the map that we will describe now. The given map ${ }_{i} \varphi_{j}:{ }_{i} M_{j} \otimes{ }_{j} X \rightarrow{ }_{i} X$ gives by adjointness a map ${ }_{j} X \rightarrow \operatorname{Hom}_{F_{i}}\left({ }_{i} M_{j},{ }_{i} X\right)$. Let then $f \in \operatorname{Hom}_{F_{i}}\left({ }_{i} X, F_{i}\right), m \in{ }_{i} M_{j}$. Then we get the following map from ${ }_{j} X$ to $F_{j}$ :

$$
{ }_{j} X \rightarrow \operatorname{Hom}_{F_{i}}\left({ }_{i} M_{j},{ }_{i} X\right) \stackrel{(\mathrm{id} f)}{\rightarrow} \operatorname{Hom}_{F_{i}}\left({ }_{i} M_{j}, F_{i}\right) \stackrel{m^{*}}{\rightarrow} F_{j},
$$

where $m^{*}$ is the image of $m$ under the bimodule isomorphism ${ }_{i} \boldsymbol{M}_{j} \rightarrow$ $\operatorname{Hom}_{F_{j}}\left(\operatorname{Hom}_{F_{i}}\left({ }_{i} M_{j}, F_{i}\right), F_{j}\right)$. There is also a natural definition on morphisms, in such a way that we get a duality $D_{1}$.

We conjecture that $C_{1}^{+}=D_{1} \mathrm{Tr}$, where $D_{1}$ is defined as above. In fact, if $\Lambda$ is an artin algebra, this turns out to be the case. To see this we use that Brenner and Butler have shown that for a particular choice of bimodule isomorphisms the corresponding Coxeter functor $C^{+}$is isomorphic to $D \mathrm{Tr}$. We recall which $C^{+}$they consider in [10]. With the previous notation we assume that the hereditary artin tensor ring $\Lambda$ is an algebra over the field $K$, 
and we fix $F_{i}$-bimodule isomorphisms $t_{i}: \operatorname{Hom}_{K}\left(F_{i}, K\right) \rightarrow F_{i}$. Consider the natural induced isomorphism

$$
\operatorname{Hom}_{K}\left({ }_{i} M_{j}, K\right) \rightarrow \operatorname{Hom}_{F_{i}}\left({ }_{i} M_{j}, \operatorname{Hom}_{K}\left(F_{i}, K\right)\right) \rightarrow \operatorname{Hom}_{F_{i}}\left({ }_{i} M_{j}, F_{i}\right)
$$

and $\operatorname{Hom}_{K}\left({ }_{i} M_{j}, K\right) \rightarrow \operatorname{Hom}_{F_{j}}\left({ }_{i} M_{j}, F_{j}\right)$. Then the Coxeter functor defined using the induced isomorphisms $\operatorname{Hom}_{F_{i}}\left({ }_{i} M_{j}, F_{i}\right) \rightarrow \operatorname{Hom}_{F_{j}}\left({ }_{i} M_{j}, F_{j}\right)$ is isomorphic to $D \operatorname{Tr}[10]$.

It is not hard to see that the duality determined by the above bimodule isomorphisms is in fact isomorphic to the ordinary duality $D$ for artin algebras. Since changing a bimodule isomorphism amounts to composing $D \mathrm{Tr}$ and $\mathrm{C}^{+}$with the same equivalence of categories, we have the claimed result for artin algebras.

We point out that for hereditary tensor rings with duality conditions, the collection of isomorphism classes of functors of the form $D_{1} \operatorname{Tr}$ for some duality $D_{1}$ can be larger than the collection of isomorphism classes of functors $C_{1}^{+}$. This will be the case for $\Lambda$ if there is an isomorphism $t: \Lambda \rightarrow \Lambda$ such that the induced equivalence $t^{*}: \bmod \Lambda \rightarrow \bmod \Lambda$ is not the identity on simples, because if $C_{1}^{+}$and $C_{2}^{+}$are Coxeter functors then $C_{1}^{+}(A)$ and $C_{2}^{+}(A)$ clearly have the same composition factors. For example, consider a field $K$ and the ring

$$
\Lambda=\left(\begin{array}{lll}
K & 0 & 0 \\
K & K & 0 \\
K & 0 & K
\end{array}\right),
$$

and let $t: \Lambda \rightarrow \Lambda$ be given by

$$
t\left(\begin{array}{lll}
a & 0 & 0 \\
b & c & 0 \\
d & 0 & e
\end{array}\right)=\left(\begin{array}{lll}
a & 0 & 0 \\
d & e & 0 \\
b & 0 & c
\end{array}\right)
$$

It is not hard to see that $t$ is a ring isomorphism with the required property.

We also point out that for any duality $D_{1}$ there is an isomorphism $s$ : $\Lambda_{n} \rightarrow \Lambda$ (in the notation of $\S 3$ ) such that $D_{1} \operatorname{Tr}$ is isomorphic to a composition of partial Coxeter functors $F_{n} \cdots F_{1}$ with the equivalence of categories $\bmod \Lambda_{n} \rightarrow \bmod \Lambda$ induced by $s$. This follows from Theorem 3.6. Further we point out that in the more general case of an hereditary ring with a duality $D_{1}$, we still have the functors $D_{1} \operatorname{Tr}: \bmod \Lambda \rightarrow \bmod \Lambda$, even though the Coxeter functors $C^{+}$may not be defined, since their definition is dependent on a diagramatic description of the rings. In this case, $D_{1} \operatorname{Tr}$ still has the property that it takes projectives to zero and induces an equivalence of categories $\bmod _{P} \Lambda \rightarrow \bmod _{I} \Lambda$.

More generally, for an artin ring $\Lambda$, not necessarily hereditary, with some duality $D_{1}, D_{1} \operatorname{Tr}$ is still defined on objects, but is not in general an equivalence between $\bmod _{P} \Lambda$ and $\bmod _{I} \Lambda$, but between $\bmod \Lambda$ and $\overline{\bmod } \Lambda$. 
For an artin algebra $\Lambda$, the ordinary duality $D$ plays a particularly important role in the theory of almost split sequences, since all almost split sequences are of the form $0 \rightarrow D \operatorname{Tr} C \rightarrow E \rightarrow C \rightarrow 0$. If $\Lambda$ is an artin ring, not much is known about almost split sequences unless $\Lambda$ is of finite type. It would be interesting to know when almost split sequences exist for hereditary tensor rings with duality conditions, and if when they do there is a particular duality $D_{1}$ such that the almost split sequences are of the form $0 \rightarrow D_{1} \operatorname{Tr} C \rightarrow$ $E \rightarrow C \rightarrow 0$. We point out that Ringel has shown that there are hereditary tensor rings with duality conditions $\Lambda$ having an indecomposable module $C$ in $\bmod \Lambda$ such that there is no almost split sequence $0 \rightarrow A \rightarrow B \rightarrow C \rightarrow 0$ in $\bmod \Lambda$ (see [20]).

To get some more information about what the hereditary tensor rings with duality conditions look like, we prove the following preliminary result.

LEMMA 5.6. Let $\Lambda$ be a basic artin ring with a duality $D_{1}$ and let ${ }_{\Lambda} I_{\Lambda}$ be $a$ two-sided injective $\Lambda$-module such that $D_{1}$ is isomorphic to $\operatorname{Hom}_{\Lambda}(, I)$ (see [1, Chapter 6]). Let $\Lambda / \mathbf{r}=F_{1} \times \cdots \times F_{n}$, and let $\mathbf{r} / \mathbf{r}^{2}=\amalg_{i} M_{j}$ be a decomposition into indecomposable bimodules, with notation as before. If $\operatorname{soc}(I)$ and $\Lambda / \mathbf{r}$ are isomorphic bimodules, then $\operatorname{Hom}_{F_{i}}\left({ }_{i} M_{j}, F_{i}\right)$ and $\operatorname{Hom}_{F_{j}}\left({ }_{i} M_{j}, F_{j}\right)$ are isomorphic bimodules.

Proof. Since $D_{1}=\operatorname{Hom}_{\Lambda}(, I)$ is a duality, it takes simple modules to simple modules, and consequently modules of Loewy length 2 to modules of Loewy length 2 . Hence we get that $\operatorname{soc}\left({ }_{\Lambda} I\right)=\operatorname{Hom}_{\Lambda}(\Lambda / r, I)$ is a semisimple $\Lambda^{\mathrm{op}}$-module, and consequently $\operatorname{soc}\left({ }_{\Lambda} I\right)=\operatorname{soc}\left(I_{\Lambda}\right)$. Similarly, $\operatorname{Hom}_{\Lambda}\left(\Lambda \Lambda / \mathrm{r}^{2},{ }_{\Lambda} I\right)$ is isomorphic as a bimodule to the submodule $L$ of $I$ consisting of those elements $y$ in $I$ such that $r^{2} y=0$. Since $\Lambda / r^{2}$ has Loewy length 2 , then $D_{1}\left(\Lambda / \mathrm{r}^{2}\right)=\operatorname{Hom}_{\Lambda}\left(\Lambda / \mathrm{r}^{2},{ }_{\Lambda} I\right)$ is a $\Lambda^{\mathrm{op}}$-module of Loewy length 2. Thus $L$ is contained in the submodule $L^{\prime}$ of $I$ consisting of those elements $y^{\prime}$ such that $y^{\prime} r^{2}=0$. With a dual argument we prove that $L^{\prime} \subset L$, so $L=L^{\prime}$ and we have then a natural isomorphism $\operatorname{Hom}_{\Lambda}\left({ }_{\Lambda} \Lambda / \mathbf{r}^{2},{ }_{\Lambda} I\right) \rightarrow$ $\operatorname{Hom}_{\Lambda}\left(\Lambda / r_{\Lambda}^{2}, I_{\Lambda}\right)$. Consider now the exact sequence of bimodules $0 \rightarrow r / r^{2} \rightarrow$ $\Lambda / r^{2} \rightarrow \Lambda / \mathbf{r} \rightarrow 0$. This gives rise to the exact sequences

$$
0 \rightarrow \operatorname{Hom}\left({ }_{\Lambda} \Lambda / \mathbf{r},{ }_{\Lambda} I\right) \rightarrow \operatorname{Hom}\left({ }_{\Lambda} \Lambda / \mathbf{r}^{2},{ }_{\Lambda} I\right) \rightarrow \operatorname{Hom}\left({ }_{\Lambda} \mathbf{r} / \mathbf{r}^{2},{ }_{\Lambda} I\right) \rightarrow 0
$$

and

$$
0 \rightarrow \operatorname{Hom}\left(\Lambda / \mathbf{r}_{\Lambda}, I_{\Lambda}\right) \rightarrow \operatorname{Hom}\left(\Lambda / r_{\Lambda}^{2}, I_{\Lambda}\right) \rightarrow \operatorname{Hom}\left(\mathbf{r} / \mathbf{r}_{\Lambda}^{2}, I_{\Lambda}\right) \rightarrow 0 .
$$

From the above remarks we get induced an isomorphism of bimodules between $\operatorname{Hom}_{F_{i}}\left({ }_{i} M_{j}, F_{i}\right)$ and $\operatorname{Hom}_{F_{j}}\left({ }_{i} M_{j}, F_{j}\right)$.

For hereditary tensor rings we now get the following description of when the duality condition holds. 
Proposition 5.7. Let $\Lambda$ be an artin hereditary tensor ring. Then $\Lambda$ satisfies the duality conditions if and only if there is a duality $D_{1}: \bmod \Lambda \rightarrow \bmod \Lambda^{\text {op }}$ such that $D_{1}\left({ }_{\Lambda} \Lambda / \mathbf{r}\right)$ and $\Lambda / \mathbf{r}$ are isomorphic bimodules.

Proof. Assume that $\Lambda$ satisfies the duality conditions. For fixed bimodule isomorphisms between the ${ }_{i} M_{j}$ let $D_{1}$ be the duality after Corollary 5.5. Then $D_{1}\left(F_{i}\right)=\operatorname{Hom}_{F_{i}}\left(F_{i}, F_{i}\right)$ and $F_{i}$ are isomorphic bimodules, so $D_{1}\left({ }_{\Lambda} \Lambda / \mathbf{r}\right)$ and $\Lambda / \mathbf{r}$ are isomorphic bimodules.

Assume conversely that $D_{1}\left({ }_{\Lambda} \Lambda / \mathbf{r}\right)$ and $\Lambda / \mathbf{r}$ are isomorphic bimodules. Write $D_{1}=\operatorname{Hom}_{\Lambda}(, I)$, for a two-sided injective $\Lambda$-module $I . D_{1}\left({ }_{\Lambda} \Lambda / \mathrm{r}\right)=$ $\operatorname{soc}_{\Lambda}(I)$, so $\operatorname{soc}_{\Lambda}(I)$ and $\Lambda / \mathbf{r}$ are isomorphic bimodules. Our desired result then follows from Lemma 5.6.

It is proven in [9], [13] that the composition factors for the partial Coxeter functors applied to modulus are given by the symmetries of the Weyl group. We end this section with a direct proof of this fact, proving that this is the case for partial Coxeter functors defined on an hereditary algebra $\Lambda$, even when $\Lambda$ is not a tensor algebra.

Let $\Lambda$ be an hereditary artin algebra and let $S_{1}, \ldots, S_{n}$ be an admissible indexing of the simples of $\Lambda$. Let $\operatorname{Gr} \Lambda$ denote the Grothendieck group of $\Lambda$ and let $[M]$ denote the class of the module $M$ in $\mathrm{Gr} \Lambda$. There is a bilinear form $B: \operatorname{Gr} \Lambda \times \operatorname{Gr} \Lambda \rightarrow Q$, where $Q$ denotes the field of rational numbers, defined as follows. We assume that $\Lambda$ is indecomposable, so the center of $\Lambda$ is a field $K$. Then

$$
\begin{aligned}
B([M],[N])=\frac{1}{2}\left(\operatorname{dim}_{K}(M, N)+\operatorname{dim}_{K}(N, M)\right. & \\
& \left.-\operatorname{dim}_{K} \operatorname{Ext}_{\Lambda}^{1}(M, N)-\operatorname{dim}_{K} \operatorname{Ext}_{\Lambda}^{1}(N, M)\right) .
\end{aligned}
$$

(See [20, §2], [3, §3].) Gr $\Lambda$ is a free abelian group, and we fix the basis [ $\left.S_{1}\right]$, $\left[S_{2}\right], \ldots,\left[S_{n}\right]$. We will then identify $\operatorname{Gr} \Lambda$ with $Z^{n}$, where $Z$ denotes the ring of integers. One can then uniquely extend $B$ to a map from $Q^{n} \times Q^{n}$ to $Q$. Let $\sigma_{i}: Q^{n} \rightarrow Q^{n}$ be the symmetry defined by

$$
\sigma_{i}(x)=x-2 \frac{B\left(x,\left[S_{i}\right]\right)}{B\left(\left[S_{i}\right],\left[S_{i}\right]\right)}\left[S_{i}\right]
$$

Let $P_{i}$ denote the projective cover for $S_{i}$, and let as in $\S 1 X=$ $\operatorname{Tr} D S \amalg P_{2} \amalg \cdots \amalg P_{n}, \Gamma=\operatorname{End}_{\Lambda}(X)^{\mathrm{op}}$ and $F=(X):, \bmod \Lambda \rightarrow \bmod \Gamma$. We now prove the following result.

Proposition 5.8. We keep the above notations. We identify $\mathrm{Gr} \Lambda$ and $\mathrm{Gr} \Gamma$ by means of the map transforming the basis $\left[S_{1}\right], \ldots,\left[S_{n}\right]$ of $\mathrm{Gr} \Lambda$ onto the basis $\left[F\left(\operatorname{Tr} D S_{1}\right) / \mathbf{r} F\left(\operatorname{Tr} D S_{1}\right)\right],\left[F\left(P_{2}\right) / \mathbf{r} F\left(P_{2}\right)\right], \ldots,\left[F\left(P_{n}\right) / \mathbf{r} F\left(P_{n}\right)\right]$. Then $[F(M)]=\sigma_{1}([M])$. 
Proof. We assume that $\Lambda$ is an indecomposable algebra over the field $K$. It is easy to see that we have the following formula:

$$
[M]=\sum_{i=1}^{n} \frac{\operatorname{dim}_{K}\left(P_{i}, M\right)}{\operatorname{dim}_{K}\left(S_{i}, S_{i}\right)}\left[S_{i}\right]
$$

We know from Proposition 2.1 that the indecomposable projective $\Lambda$-modules are $F\left(\operatorname{Tr} D S_{1}\right), F\left(P_{2}\right), \ldots, F\left(P_{n}\right)$, and that $T=F\left(\operatorname{Tr} D S_{1}\right) / \mathbf{r} F\left(\operatorname{Tr} D S_{1}\right)$ is simple injective.

Since $\operatorname{Tr} D: \bmod _{I} \Lambda \rightarrow \bmod _{P} \Lambda$ is an equivalence of categories and $S_{1}$ is not injective we have that $\operatorname{dim}_{K}\left(S_{1}, S_{1}\right)=\operatorname{dim}_{K}\left(\operatorname{Tr} D S_{1}, \operatorname{Tr} D S_{1}\right)=$ $\operatorname{dim}_{K}\left(F\left(\operatorname{Tr} D S_{1}\right), F_{1}\left(\operatorname{Tr} D S_{1}\right)\right)=\operatorname{dim}_{K}(T, T)$.

On the other hand, $\operatorname{dim}_{K}\left(F\left(P_{i}\right), F\left(P_{i}\right)\right)=\operatorname{dim}_{K}\left(P_{i}, P_{i}\right)$ for $i=2, \ldots, n$. Hence we have

$$
\begin{aligned}
{[F(M)]=} & \sum_{i=2}^{n} \frac{\operatorname{dim}_{K}\left(F\left(P_{i}\right), F(M)\right)}{\operatorname{dim}_{K}\left(F\left(S_{i}\right) / \mathbf{r} F\left(S_{i}\right), F\left(S_{i}\right) / \mathbf{r} F\left(S_{i}\right)\right)}\left[S_{i}\right] \\
& +\frac{\operatorname{dim}_{K}\left(F\left(\operatorname{Tr} D S_{1}\right), F(M)\right)}{\operatorname{dim}_{K}(T, T)}\left[S_{1}\right] \\
= & \sum_{i=1}^{n} \frac{\operatorname{dim}_{K}\left(P_{i}, M\right)}{\operatorname{dim}_{K}\left(S_{i}, S_{i}\right)}\left[S_{i}\right]+\frac{\operatorname{dim}_{K}\left(\operatorname{Tr} D S_{1}, M\right)}{\operatorname{dim}_{K}\left(S_{1}, S_{1}\right)}\left[S_{1}\right]
\end{aligned}
$$

Hence

$$
[F(M)]-[M]=\frac{\operatorname{dim}_{K}\left(\operatorname{Tr} D S_{1}, M\right)-\operatorname{dim}_{K}\left(S_{1}, M\right)}{\operatorname{dim}_{K}\left(S_{1}, S_{1}\right)}\left[S_{1}\right]
$$

Using that $D\left(\operatorname{Tr} D S_{1}, M\right)=\operatorname{Ext}_{\Lambda}^{1}\left(M, S_{1}\right)($ see [6, Theorem 3.3], [3,\&1]) we have that

$$
[F(M)]=[M]-\frac{B\left(\left[S_{1}\right],[M]\right)}{B\left(\left[S_{1}\right],\left[S_{1}\right]\right)}\left[S_{1}\right]=\sigma_{1}(M)
$$

This finishes the proof of the proposition.

Note. In the above proposition we dealt with the functor $F=(X$,$) . Using$ the results of the end of $\$ 2$ about the uniqueness of the partial Coxeter functor up to equivalence, one can prove that Proposition 5.8 holds for an arbitrary partial Coxeter functor $F$. But we observe that this does not mean that if $F, F^{\prime}: \bmod \Lambda \rightarrow \bmod \Gamma$ are two partial Coxeter functors, then $F(M)$ and $F^{\prime}(M)$ have the same composition factors. It means that there is a permutation $\beta$ of the simples of $\Lambda$ such that if $[F(M)]=\sum_{i=1}^{n} n_{i}\left[S_{i}\right]$, then $\left[F^{\prime}(M)\right]=\sum_{i=1}^{n} n_{i}\left[\beta\left(S_{i}\right)\right]$. 


\section{REFERENCES}

1. F. W. Anderson and K. R. Fuller, Rings and categories of modules, Graduate Texts in Mathematics, no. 13, Springer-Verlag, Berlin and New York, 1974.

2. M. Auslander, Representation theory of artin algebras. I, Comm. Algebra 1 (1974), 177-268.

3. M. Auslander and M. I. Platzeck, Representation theory of hereditary artin algebras, Lecture Notes in Pure and Appl. Math., vol. 37 Dekker, New York, 1978.

4. M. Auslander and I. Reiten, Notes on representation theory of artin algebras, Brandeis Univ., 1972.

5. Stable equivalence of artin algebras, Lecture Notes in Math., vol. 353, SpringerVerlag, Berlin and New York, 1973.

6. __ Representation theory of artin algebras. III, Almost split sequences, Comm. Algebra 3 (1975), 239-294.

7.

8. R. Bautista, Algebras close to hereditary algebras (to appear).

9. I. N. Bernstein, I. M. Gelfand and V. A. Ponomarev, Coxeter functors and a theorem of Gabriel, Uspehi Mat. Nauk 28 (1973), 19-33 = Russian Math. Surveys 28 (1973), 17-32.

10. S. Brenner and M. C. R. Butler, The equivalence of certain functors occurring in the representation theory of artin algebras and species, J. London Math. Soc. 14 (1976), 183-187.

11. E. Cartan and S. Eilenberg, Homological algebra, Princeton Univ. Press, Princeton, N. J., 1956.

12. V. Dlab and C. M. Ringel, Representations of graphs and algebras, J. Algebra 33 (1975), 306-394.

13. __ Representations of graphs and algebras, Mem. Amer. Math. Soc., No. 173 (1976).

14. __ Representation theory of algebras, Lecture Notes in Pure and Appl. Math., vol. 37, Dekker, New York, 1978.

15. C. Faith, Algebra: Rings, modules and categories. I, Die Grundlehren der math. Wissenschaften, Band 190, Springer-Verlag, Berlin and New York, 1973.

16. R. Fossum, P. Griffith and I. Reiten, Trivial extensions of abelian categories, Lecture Notes in Math., vol. 456, Springer-Verlag, Berlin and New York, 1975. 444.

17. P. Gabriel, Répréséntations indécomposables, Séminaire Bourbaki, 26e année, (1973/74), no.

18. E. Green, The representation theory of tensor algebras, J. Algebra 34 (1975), 136-171.

19. M. Harada, Hereditary semiprimary rings and triangular matrix rings, Nagoya Math. J. 27 (1966), 463-484.

20. C. M. Ringel, Representations of $K$-species and bimodules, J. Algebra 41 (1976), 269-302.

Department of Mathematics, Brandeis University, Waltham, Massachusetts 02154 (Current address of M. Auslander)

Department of Mathematics, University of Illinois, Urbana, IllnNois 61801

Department of Mathematics, University of Trondhem, NLHT, 7000 Trondhem, NoRWAY (Current address of I. Reiten)

Current address (M. I. Platzeck): Corrientes $211.9^{\circ} \mathrm{B}, 8000$ Bahia Blanca, Argentina 